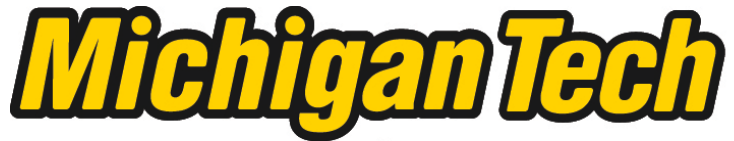 \\ Michigan Technological University Create the Future Digital Commons @ Michigan Tech
}

\section{Direct Write Fabrication of Waveguides and Interconnects for Optical Printed Wiring Boards}

Joseph Carl Dingeldein

Michigan Technological University

Follow this and additional works at: https://digitalcommons.mtu.edu/etds

Part of the Mechanical Engineering Commons, and the Optics Commons Copyright 2012 Joseph Carl Dingeldein

\section{Recommended Citation}

Dingeldein, Joseph Carl, "Direct Write Fabrication of Waveguides and Interconnects for Optical Printed Wiring Boards", Dissertation, Michigan Technological University, 2012.

https://doi.org/10.37099/mtu.dc.etds/481

Follow this and additional works at: https://digitalcommons.mtu.edu/etds

Part of the Mechanical Engineering Commons, and the Optics Commons 


\title{
DIRECT WRITE FABRICATION OF WAVEGUIDES AND INTERCONNECTS FOR OPTICAL PRINTED WIRING BOARDS
}

\author{
By \\ Joseph C. Dingeldein

\begin{abstract}
A DISSERTATION
Submitted in partial fulfillment of the requirements for the degree of DOCTOR OF PHILOSOPHY

(Mechanical Engineering - Engineering Mechanics)
\end{abstract}

MICHIGAN TECHNOLOGICAL UNIVERSITY

2012

(C)2012 Joseph C. Dingeldein 
This dissertation, "Direct Write Fabrication of Waveguides and Interconnects for Optical Printed Wiring Boards," is hereby approved in partial fulfillment of the requirements for the Degree of DOCTOR OF PHILOSOPHY IN MECHANICAL ENGINEERINGENGINEERING MECHANICS

Department of Mechanical Engineering-Engineering Mechanics

Signatures:

Dissertation Advisor

Dr. Craig Friedrich

Department Chair

Dr. William Predebon

Date 
Things may come to those who wait, but only the things left by those who hustle. -Abraham Lincoln

This work is dedicated to my father for walking plainly in life and showing me how to be a good man, and also to my amazing wife, who is my joy and inspiration. 


\section{TABLE OF CONTENTS}

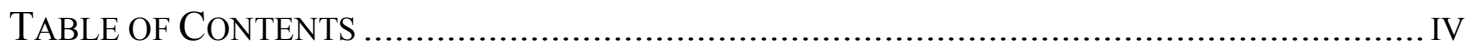

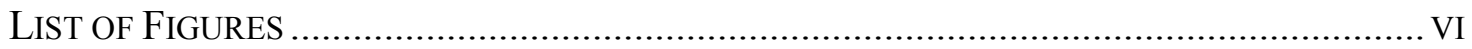

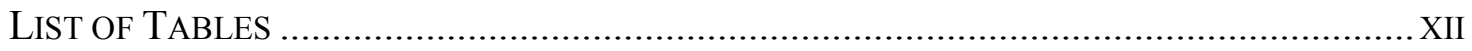

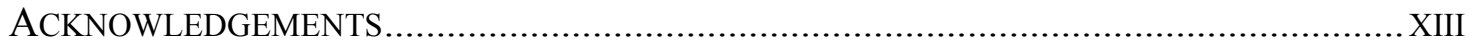

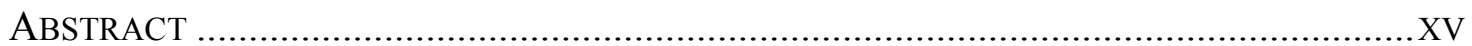

CHAPTER 1. INTRODUCTION ...................................................................... 1

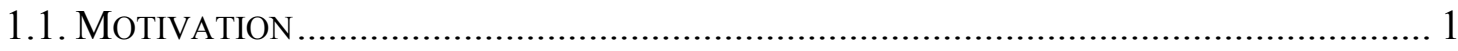

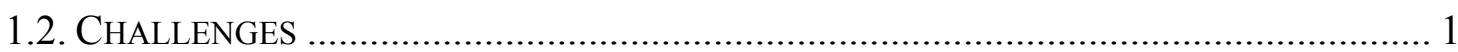

CHAPTER 2. LITERATURE REVIEW .................................................................... 3

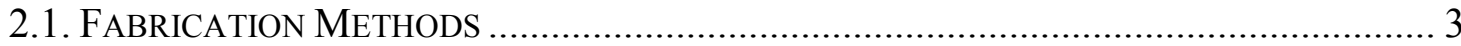

2.1.1. Material Application Methods .................................................................... 3

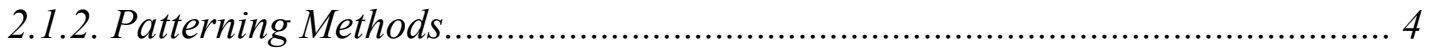

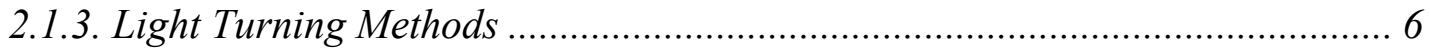

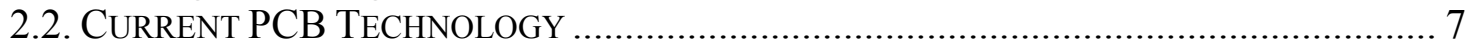

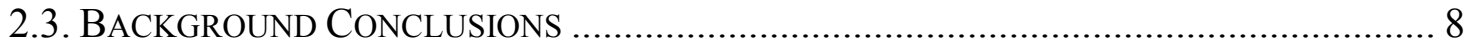

CHAPTER 3. DIRECT WRITE WAVEGUIDE FABRICATION TOOL ................9

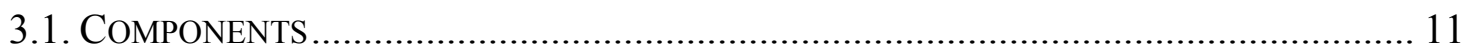

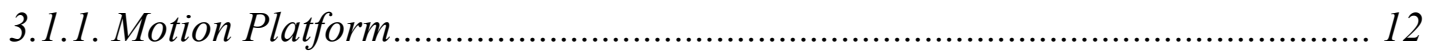

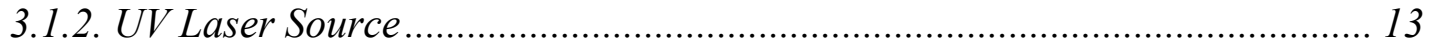

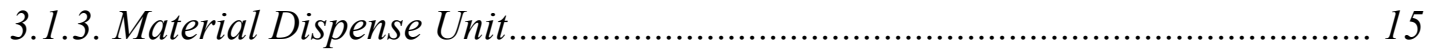

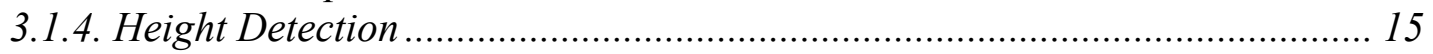

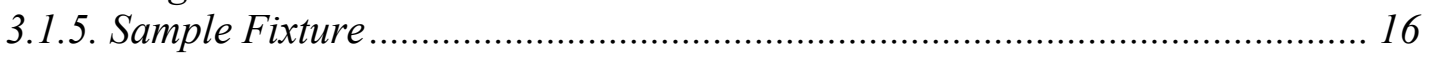

CHAPTER 4. LASER DIRECT WRITE PHOTO-PATTERNING ........................... 17

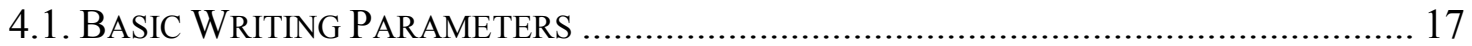

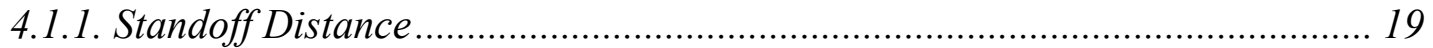

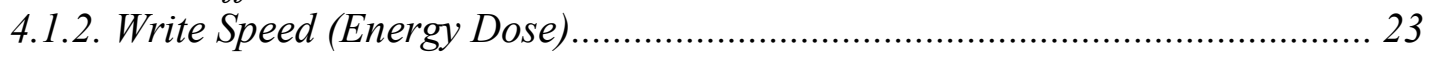

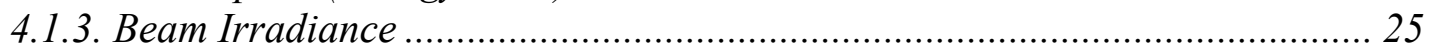

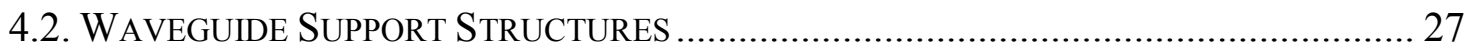

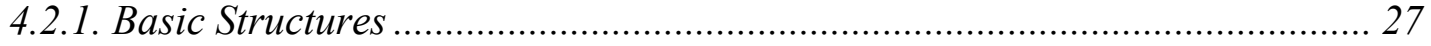

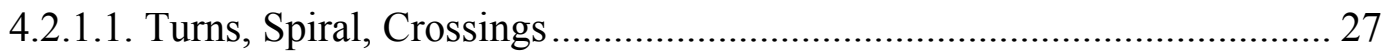

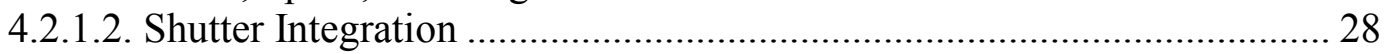

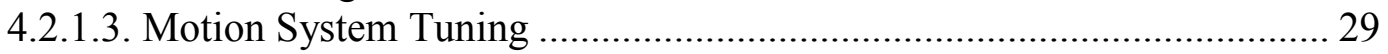

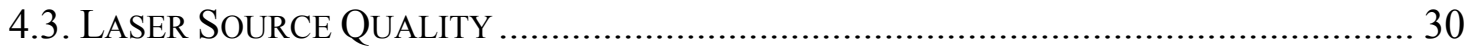

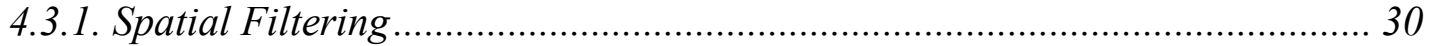

4.3.2. Single Mode Fiber Coupled Laser for Beam Clean-up ............................... 32

4.3.3. Optical Via Structures.............................................................................. 36

4.3.3.1. Angled Waveguides for Reflector ................................................... 36

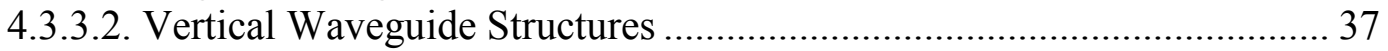


4.4. LASER Direct Write Photo-PATterning CONCLUSIONS ................................... 38

CHAPTER 5. DIRECT DISPENSE MATERIAL DEPOSITION........................... 39

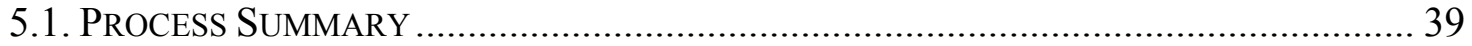

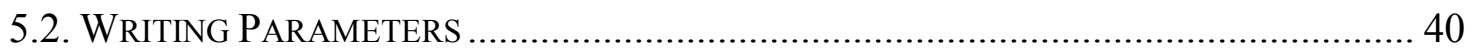

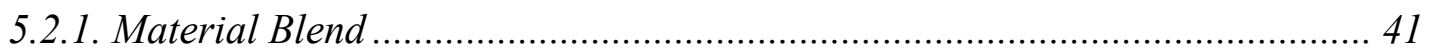

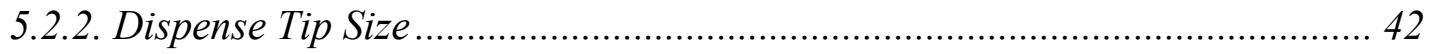

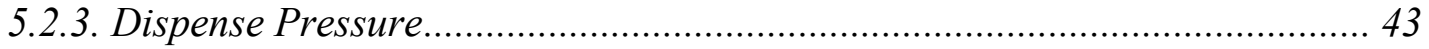

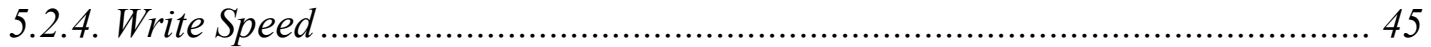

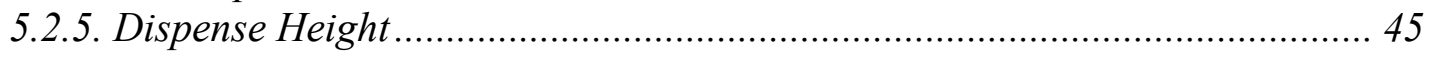

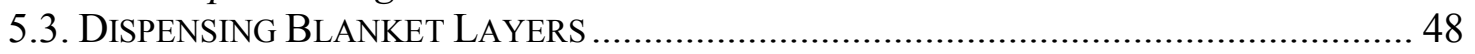

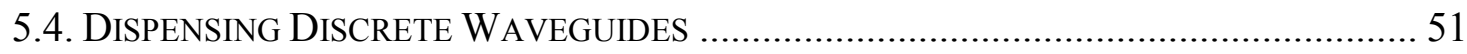

5.5. DiRECT DiSPENSE CONCLUSIONS AND FUTURE WORK ...................................... 55

\section{CHAPTER 6. OPTICAL ELECTRICAL PRINTED WIRING BOARD}

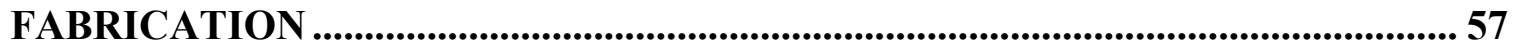

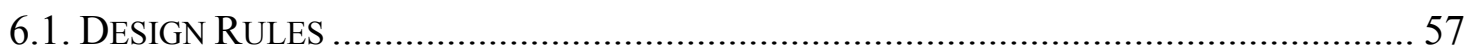

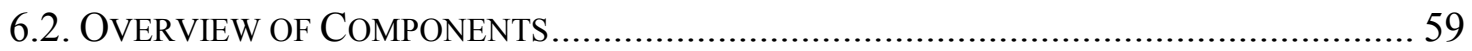

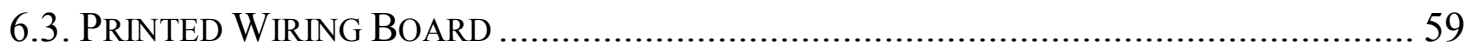

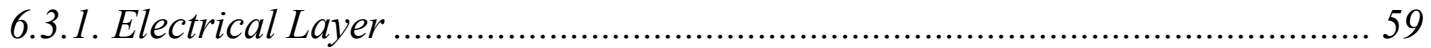

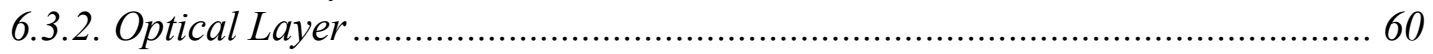

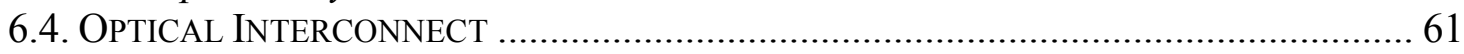

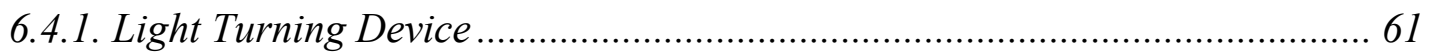

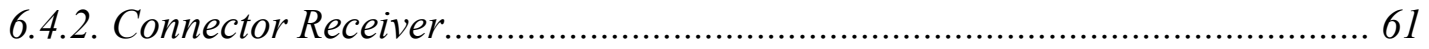

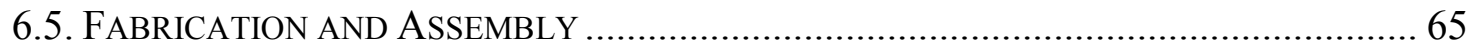

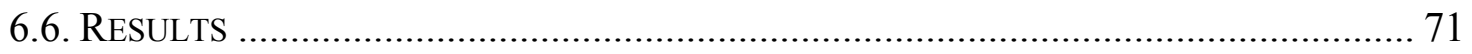

6.6.1. Initial Characterization ........................................................................ 71

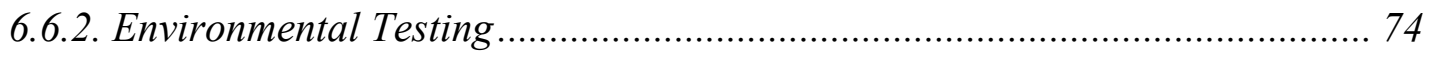

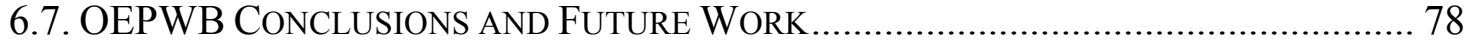

6.7.1. Acceptance Test Plan for Manufacturing ................................................. 79

CHAPTER 7. CONCLUSION AND RECOMMENDATION................................... 82

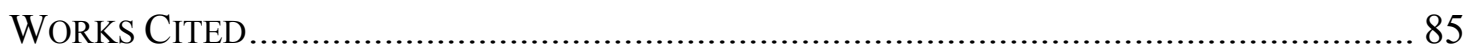

APPENDIX A: SUMMARY TABLE OF MTU LASER WRITING PARAMETERS AND RESULTS 91

APPENDIX B: PERMISSIONS FROM PUBLISHERS. 


\section{LIST OF FIGURES}

Figure 2.1. Molding, or "groove-filling" method (left) and embossing, or "rib-cladding" method (right) of fabricating waveguides.................................................................. 5

Figure 2.2. Profiles of waveguide end faces created by way of (A) micro milling, dicing, and reactive ion etching, (B) dicing, and (C) laser ablation....................................... 6

Figure 3.1. General photolithographic processing steps............................................... 10

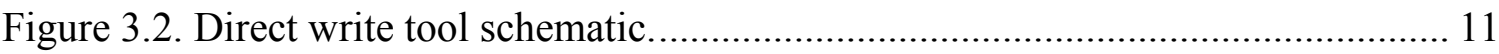

Figure 3.3. Direct write tool platform with mounted laser and syringe dispenser, along with vacuum sample holder. ............................................................................... 12

Figure 3.4. The effect of waveguide sidewall roughness on optical performance [8]..... 13

Figure 3.5. UV-Vis absorbance spectrum of Dow Corning OE4100 waveguide material (reproduced with permission from Dow Corning which can be found in Appendix

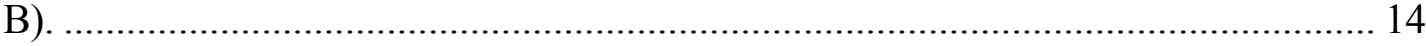

Figure 3.6. Syringe assembly is loosely mounted in fixture to allow safe contact with the

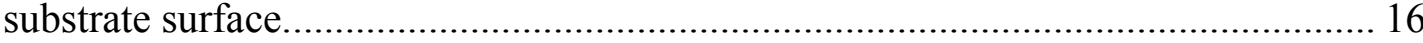

Figure 4.1. Standoff distance defines the location of sample within the beam path, which influences pot size. Writing at the beam waist provides minimum lateral dimensions and maximum dose per time.............................................................................. 17

Figure 4.2. Effect of Gaussian power distribution on waveguide width. Slower write speeds lead to larger features................................................................................ 18

Figure 4.3. Effect of increasing beam irradiance on curing process................................ 19

Figure 4.4. Layout of parameter array for direct write sample ....................................... 20

Figure 4.5. Beam scan collage captured for $5 \mathrm{~mW}$ laser output power depicts beam cross sections at standoff distances of $37-50 \mathrm{~mm}$. Scans are used to identify the standoff distance of the beam waist, which falls between $46-48 \mathrm{~mm}$.

Figure 4.6. Cross sectional views of waveguides showing effect of standoff distance (at

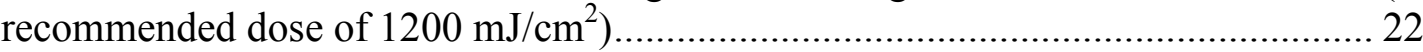

Figure 4.7. Relationship between standoff distance and resulting waveguide width for each of the six dose amounts examined (manufacturer recommended dose is 1200 $\mathrm{mJ} / \mathrm{cm}^{2}$ ).

Figure 4.8. Relationship between energy dose amount and resulting waveguide width for

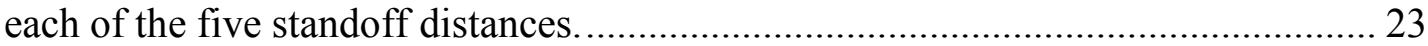

Figure 4.9. Cross sectional views of waveguides written along the major and minor axis of the slightly elliptical beam. The observed trends are the same for each axis, but the specific waveguide dimensions are not uniform between axes. 
Figure 4.10. Laser output power density characterization experiment setup. Samples were written with a constant energy dose amount $\left(100 \%\right.$ or $\left.1200 \mathrm{~mJ} / \mathrm{cm}^{2}\right)$........... 25

Figure 4.11. Relationship between laser output power and resulting waveguide width for each of the standoff distances for a fixed energy dose amount. Less than $10 \mu \mathrm{m}$ deviation between guide widths over a power range from $7-17 \mathrm{~mW}$. 26

Figure 4.12. Cross sectional views of waveguides showing effect of output power density of laser. 26

Figure 4.13. Laser output power plot that shows a discrepancy between actual output power and that seen on the laser GUI interface, especially at the lower power levels $(<5 \mathrm{~mW})$. 27

Figure 4.14. CAD drawings for waveguide exposure patterns. $90^{\circ}$ turns, $1-10 \mathrm{~mm}$ radius with 500 um pitch (left), $250 \mathrm{um}$ pitch (center), and $1 \mathrm{~m}$ spiral with $30 \mathrm{~mm}$ maximum

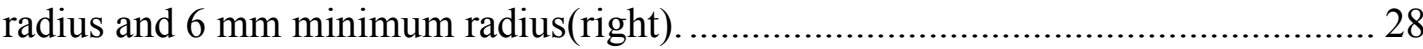

Figure 4.15. Crossing pattern shows that some standard CNC system features are not ideal for waveguide patterning. General operation requires system to decelerate to zero velocity between movements, over-curing the material. 29

Figure 4.16. Shutter operates in conjunction with motion platform to prevent over-curing

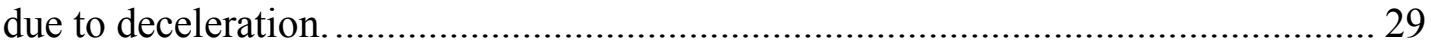

Figure 4.17. Proper system tuning is critical to waveguide performance. The system on the left is under-damped, resulting in oscillating waveguides, while the system on the right is properly tuned. 30

Figure 4.18. Spatial filter schematic and resulting output. Ring patterns have been removed and elliptical beam shape is now circular with more uniform power distribution. 31

Figure 4.19. Lab setup of laser and spatial filter required a lot of space and was very labor intensive to achieve proper alignment between all of the components. 31

Figure 4.20. A Toptica iBeam Smart 375-S UV laser $(18 \mathrm{~mW}, 379 \mathrm{~nm}$ measured peak wavelength) was coupled into a UV grade single mode fiber with the Toptica SmartDock fiber coupler, making it easy to mount to the Z-axis............................... 32

Figure 4.21. Beam divergence necessitates short standoff distance. ................................. 33

Figure 4.22. SMF coupled laser with collimator provides best direct write results, second overall to lithographically produced waveguides. 34

Figure 4.23. Beam outputs for laser diode, single mode fiber coupled source, and lensed output. 35

Figure 4.24. Waveguides patterned at a standoff distance of $1 \mathrm{~mm}$ and a feed rate of 57

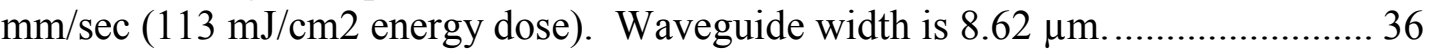

Figure 4.25. Angled waveguide structure (left) serves as reflector face for orthogonal

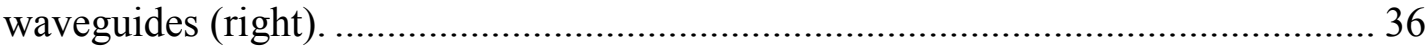


Figure 4.26. SEM images of spot cured waveguide material to form high aspect ratio

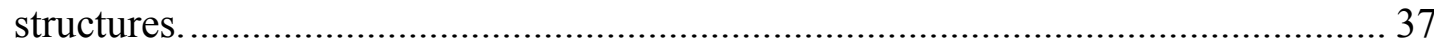

Figure 4.27. Aberrations in beam negatively affect patterning process. ........................ 38

Figure 5.1. Steady flow diagram of dispense process. Material flows from a large reservoir out through a small diameter needle, increasing in flow velocity along the way. 40

Figure 5.2. Core material with non-volatile contents of $70,75,80,85$, and $90 \%$ currently

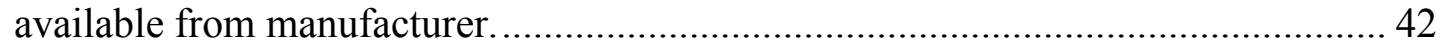

Figure 5.3. 33 gauge $(210 \mu \mathrm{m}$ outer diameter, $110 \mu \mathrm{m}$ inner diameter $)$ stainless steel needle tip, side view (left, 50x) and end view (right, 100x)...... 43

Figure 5.4. Pre-pulled glass needle dispense tips: $30 \mu \mathrm{m}$ inner diameter (left) and $2 \mu \mathrm{m}$ inner diameter (right).

Figure 5.5. Flow rates of glycerol, core, and clad material through a 21 gauge needle as a function of applied pressure.

Figure 5.6. Flow rate of core material through a 32 gauge needle as a function of applied pressure. 45

Figure 5.7. Dispense height of 75 um was too high from substrate, resulting in periodic bead patterns. 46

Figure 5.8. Repetitive cycle observed in the dispensing process when the dispense needle is positioned too high off the substrate surface: (A) droplet forms on tip of needle (B) droplet grows in size until it contacts the substrate surface (C) droplet adheres to substrate and draws material out of needle (D) material necks until the material string is drawn out and sometimes broken, after which the process begins again. 46

Figure 5.9. Dispense height of 50 um was too close to the substrate, resulting in flat and streaked waveguides.

Figure 5.10. Dispense needle is too close to substrate surface, inhibiting free flow from the tip

Figure 5.11. Dispense height of 65 um resulted in continuous and uniform waveguide

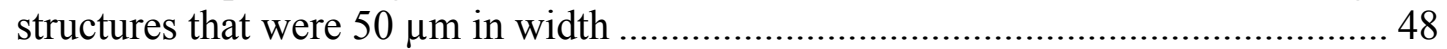

Figure 5.12. Layer cross sections for pressure settings of 10, 15, 20, 25, 30 PSI, from left to right. Thicknesses range from $25 \mu \mathrm{m}$ at 10 PSI to $55 \mu \mathrm{m}$ at 30 PSI for a line pitch of $0.25 \mathrm{~mm}$

Figure 5.13. Layer cross sections for pressure settings of 1.5, 2, 2.5, 3, and 3.5 PSI, from left to right. Thicknesses range from $50 \mu \mathrm{m}$ at 1.5 PSI to $220 \mu \mathrm{m}$ at 3.5 PSI for a line pitch of $1 \mathrm{~mm}$. 49

Figure 5.14. Guides written at 35 PSI (left) and 4 PSI (right) with a 32 gauge needle... 51 
Figure 5.15. Waveguide width as a function of dispense pressure for $70,75,80$, and $85 \%$ NVC core material through a 33 gauge needle................................................... 52

Figure 5.16. Waveguide height as a function of dispense pressure for $70,75,80$, and $85 \%$ NVC core material through a 33 gauge needle................................................... 52

Figure 5.17. 75\% Solid content core material dispensed through a 33 gauge needle....... 53

Figure 5.18. 80\% Solid content core material dispensed through a 33 gauge needle....... 53

Figure 5.19. 85\% Solid content core material dispensed through a 33 gauge needle....... 54

Figure 5.20. Waveguide aspect ratio (height : width) as a function of dispense pressure for $70,75,80$, and $85 \%$ NVC core material through a 33 gauge needle................... 54

Figure 5.21. Direct dispense waveguide cross sections for pressures of 65, 75, 80 PSI. . 55

Figure 5.22. Shows the dispense needle and guide pin configuration............................ 56

Figure 6.1. Optical routing schematic used for performance testing at Michigan Technological University.................................................................................. 58

Figure 6.2. (A) Optical layer with machined LTD insertion pockets (B) Electrical overlay panel (C) Complete optical assembly with electrical overlay panel..........................59

Figure 6.3. (A) Optical layer with generic optical components (B) Electrical layer overlay (C) Complete optical assembly with electrical overlay panel. 60

Figure 6.4. CAD detail of four patterned waveguide configurations for optical layer examining effect of waveguide pitch on optical performance: (A) Pitch narrows from $250 \mu \mathrm{m}$ to $100 \mu \mathrm{m}$ for all 12 waveguides (B) Pitch narrows from $250 \mu \mathrm{m}$ to $100 \mu \mathrm{m}$ for center four waveguides (C) Pitch narrows from $250 \mu \mathrm{m}$ to $100 \mu \mathrm{m}$ for center two waveguides (D) Pitch remains at standard $250 \mu \mathrm{m}$ for all 12 waveguides. 60

Figure 6.5. 12-Channel Micro Lens Array by microPEP (left) device (right) CAD representation. 61

Figure 6.6. CAD design of basic MT style connector receiver. The larger cavity accommodates the LTD, which protrudes slightly from the board surface, and the smaller slot accommodates the external MT connector. 62

Figure 6.7. MT connector receivers provide environmental protection and stability to the optical device area. 62

Figure 6.8. MTP/MPO style connection receptacles provide further environmental protection, as well as a more standardized form factor. Spring loaded compression fitting with clipping ability better suited for vibration testing..... 63

Figure 6.9. Illustrating how the LTD would snap into the modified receptacle using built in alignment features, and would then be epoxied into place at each end, away from the free space optics. 64 
Figure 6.10. Machined receptacle with inserted LTD. Back "gate" portion had to be cut

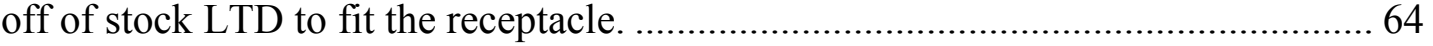

Figure 6.11. Alternate receiver design for mating with USCONEC PRIZM connector. This style offers improved form factor and ability to secure cabling for shock and vibration testing. 64

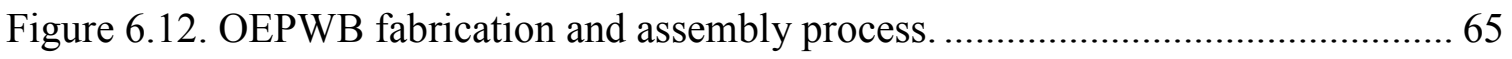

Figure 6.13. (A) Up milling puts the brittle waveguide material in tension, causing it to chip and fragment, drastically degrading optical quality (B) Down milling puts the brittle waveguide material into compression, reducing the amount of fragmentation resulting in a more uniform surface finish, where $d_{r}$ is radial depth of cut. 66

Figure 6.14. Siloxane material exhibits chipping when put in tension, due to improper feed direction (left) and improved cut quality when material is put in compression while down milling (right). 66

Figure 6.15. Milling schematic illustrating the inclusion of finish cuts at decreasing radial depths of cut $\left(d_{r}\right)$ to improve surface finish.

Figure 6.16. Manually operated alignment stages used to precisely position the LTDs during insertion into the optical layer.

Figure 6.17. Custom mounting fixture used to secure the input and output cabling for positioning with respect to the optical layer. 68

Figure 6.18. LTD is secured to the clamped MT connector for precision optical alignment with respect to the optical layer. 68

Figure 6.19. LTD insertion procedure control loop ensured proper alignment between the LTDs and the embedded optical waveguides. 69

Figure 6.20. All six degrees of freedom for the LTD were adjustable, but the three indicated parameters played the most significant role in performance. 69

Figure 6.21. Locations of UV and final, two-part epoxy deposits................................... 70

Figure 6.22. Optical component stack comprised of optical layer, LTD, connector receiver, and MT connector. 71

Figure 6.23. Cross talk measurements for each of the four waveguide groups on the three individual samples. 73

Figure 6.24. Cross talk difference between circuit isolation differences $250 \mu \mathrm{m}$ versus $100 \mu \mathrm{m}$. ANOVA statistical analysis shows that there is no significant difference in signal direction (between "send" and "receive"), as $\mathrm{P}>0.05$. There is, however, a significant difference in cross talk when comparing pitch values, as $\mathrm{P}<0.05$........ 74

Figure 6.25. Temperature humidity cycling sequence provided by Lockheed Martin..... 75 
Figure 6.26. Optical loss results for Sample A after each round of environmental testing, with all 48 connectorized waveguides represented. Failures began to increase as stages of thermal cycling became more stringent.................................................. 77

Figure 6.27. Optical loss results for Sample B after each round of environmental testing, with all 48 connectorized waveguides represented. Sample survived 100 cycles of $0^{\circ} \mathrm{C}$ to $70^{\circ} \mathrm{C}$ and temperature humidity cycling. Performance significantly decreased with accelerated aging at $110^{\circ} \mathrm{C}$.

Figure 6.28. Optical loss results for Sample C after each round of environmental testing, with all 48 connectorized waveguides represented. Sample survived 100 cycles of $40^{\circ} \mathrm{C}$ to $85^{\circ} \mathrm{C}$ and temperature humidity cycling. Performance significantly

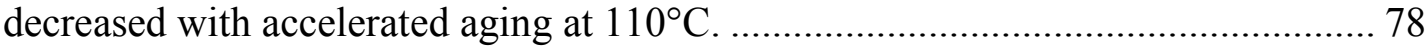

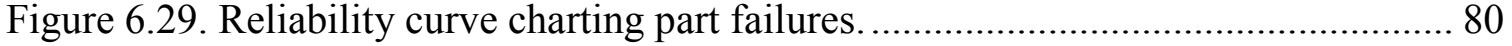

Figure 6.30. A typical Weibull plot, where the horizontal scale is a measure of life or aging (start/stop cycles, operating time, or mileage) and the vertical scale is the cumulative percentage failed [75]. Weibull Plot would be used in determining acceptable test parameters for early failure detection. 81 


\section{LIST OF TABLES}

Table 4.1. Write speeds for energy doses at a fixed laser output power of $17 \mathrm{~mW} \ldots \ldots . .20$

Table 4.2. Writing parameters for patterning $50 \mu \mathrm{m}$ wide waveguides. ......................... 24

Table 4.3. Out power and power density values based on percentage of maximum output

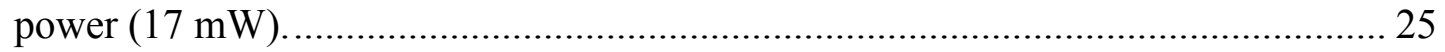

Table 5.1. Dimensions for tips used in direct dispense process..................................... 42

Table 5.2. Layer thicknesses by dispense pressure for 21 and 32 gauge needle ............. 49

Table 6.1. Power loss link budget, budgeting acceptable losses for specific design areas to ensure total losses do not exceed the $10 \mathrm{~dB}$ limit......................................................5

Table 6.2. Initial output power (and calculated optical loss) results for Samples A, B, and $\mathrm{C}$, obtained from MTU and CEC. Loss measurements calculated using $2000 \mu \mathrm{W}$

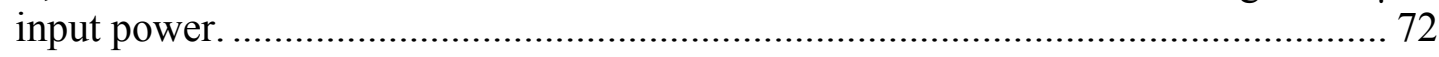

Table 6.3. Comparison between initial testing conducted by MTU/CEC and Lockheed

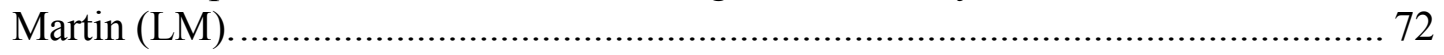

Table 6.4. Samples used for various environmental testing. ...................................... 76

Table 7.1. Laser direct writing parameters and results for MTU focused laser diode module, characterizing standoff-distance and energy dose. ................................ 91 


\section{ACKNOWLEDGEMENTS}

If there is one thing I have in spades, it is the love and support of family. I am proud to say that I have truly incredible parents, and there is no way that I would have made it this far without them. Mom and Dad, you have taught me how to endeavor, and how to be a good man. You have been supportive of me and have offered steady encouragement throughout this decade long odyssey, providing fuel for my locomotive. You have passed down character and perseverance and I know that God surely blessed me by providing me with such steady foundation. My house is built on rock.

I grew up in a house full of boys, graced with two best friends from the jump. Matt and Andy, you made me strong enough to overcome anything and I want you to share in this achievement with me, as I wouldn't be who I am without you. I also want to thank Drew and Jon for reminding me how much fun it is to have brothers while I've been away from my own family.

Thank you to the Blakes and Hagers for welcoming me into the clan and for providing an abundance of excellent food for a boy who sorely missed home cooked meals. Cindy and Toni, Scott and Amy, thank you for being so warm and supportive. I'm lucky to have married into such great family.

While my family provided support, I couldn't have accomplished this if there weren't a great many at Michigan Tech who have provided instruction as I learned my craft. Karl Walczak, I consider myself incredibly fortunate to have been able to work alongside you through my first couple years, gaining friendship and experience. You taught me the ropes and served as an excellent example while I learned of science and professionalism. You are thorough and have great attention to detail, and you are gifted with a great aptitude for problem solving. These are traits that every prospective engineer should strive for, and I will always wish you success and happiness for mentoring me.

Dr. Friedrich, thank you for providing me much the same and for offering me space to grow and learn. Your down to earth sensibility and straightforward approach to engineering have been tremendous examples for me over the years. Even if you went to that other college in Oklahoma, I consider myself beyond fortunate to have had you advise me through this process. After all, anyone who roots for the Browns must be good people. I'm honored to be your student and friend.

I would like to offer a very big thank you to the collection of individuals I've had the pleasure of working and interacting with during the HIOPTIMA project. My project colleagues, partners, and sponsors made the experience incredibly rewarding. It has been my great privilege to have learned under my committee members: Dr. Roggemann, Dr. Middlebrook, and Dr. Bergstrom. I couldn't have picked a better group of characters and intellects to guide me along, and I have enjoyed your company and wisdom over the

years. Thanks to all of my peers on the HIOPTIMA team: Casey Demars, Brandon 
Swatowski, Ben Madsen, Kevin Kruse, Nick Riegel, and Tom Daunais; it was a lot of fun working with you guys! Casey and Ben, I will always remember the days up in Cali, working in the lab and doing the Bernie. Radheshyam Tewari, I have been very fortunate to be your colleague, and I will always appreciate the many conversations we've shared. I am proud to call you my friend.

We had great partners on our project in Calumet Electronics Corporation, Lockheed Martin, and Dow Corning, and it was a great experience working with our project sponsor, NAVAIR. I'm glad to have gotten to know Stephen Vairo and Stephen Marshall at CEC, Kevin Thorson and Rick Stevens at Lockheed Martin, Dave DeShazer and Khris Alvarez at Dow Corning, and Clarence Harris, Mark Beranek, and Brian McDermott at NAVAIR.

I especially want to thank the incredible community at Michigan Tech and the Keweenaw as a whole. I've made wonderful friends in my time at MTU that I will carry with me always. I'd like to thank Joel Vertin specifically, for being such an amazing friend and support, for always encouraging me ("Gets some!"), and keeping me on point. I'm quite certain that I've been spoiled in my time here, and there is not a place on earth that is going to offer the same abundance of breathtaking scenery, outdoor activity, warm, affable people, and tasty, tasty beer. I'm very lucky to have married a Yooper, so that I might always be able to call this place home.

This brings us to the most important recipient of my gratitude, my beautiful wife. Finding you was easily the most important and rewarding success I have ever known. You fill my life with color and happiness. Thank you for being so silly and for being mine. I could not have asked for a better partner, and I'm looking forward to laughing our days away, together. God has richly blessed my life. 


\section{ABSTRACT}

Current copper based circuit technology is becoming a limiting factor in high speed data transfer applications as processors are improving at a faster rate than are developments to increase on board data transfer. One solution is to utilize optical waveguide technology to overcome these bandwidth and loss restrictions. The use of this technology virtually eliminates the heat and cross-talk loss seen in copper circuitry, while also operating at a higher bandwidth. Transitioning current fabrication techniques from small scale laboratory environments to large scale manufacturing presents significant challenges. Optical-to-electrical connections and out-of-plane coupling are significant hurdles in the advancement of optical interconnects.

The main goals of this research are the development of direct write material deposition and patterning tools for the fabrication of waveguide systems on large substrates, and the development of out-of-plane coupler components compatible with standard fiber optic cabling. Combining these elements with standard printed circuit boards allows for the fabrication of fully functional optical-electrical-printed-wiring-boards (OEPWBs).

A direct dispense tool was designed, assembled, and characterized for the repeatable dispensing of blanket waveguide layers over a range of thicknesses $(25-225 \mu \mathrm{m})$, eliminating waste material and affording the ability to utilize large substrates. This tool was used to directly dispense multimode waveguide cores which required no UV definition or development. These cores had circular cross sections and were comparable in optical performance to lithographically fabricated square waveguides.

Laser direct writing is a non-contact process that allows for the dynamic UV patterning of waveguide material on large substrates, eliminating the need for high resolution masks. A laser direct write tool was designed, assembled, and characterized for direct write patterning waveguides that were comparable in quality to those produced using standard lithographic practices $(0.047 \mathrm{~dB} / \mathrm{cm}$ loss for laser written waveguides compared to 0.043 $\mathrm{dB} / \mathrm{cm}$ for lithographic waveguides). Straight waveguides, and waveguide turns were patterned at multimode and single mode sizes, and the process was characterized and documented. Support structures such as angled reflectors and vertical posts were produced, showing the versatility of the laser direct write tool.

Commercially available components were implanted into the optical layer for out-ofplane routing of the optical signals. These devices featured spherical lenses on the input and output sides of a total internal reflection (TIR) mirror, as well as alignment pins compatible with standard MT design. Fully functional OEPWBs were fabricated featuring input and output out-of-plane optical signal routing with total optical losses not exceeding $10 \mathrm{~dB}$. These prototypes survived thermal cycling $\left(-40^{\circ} \mathrm{C}\right.$ to $\left.85^{\circ} \mathrm{C}\right)$ and humidity exposure $(95 \pm 4 \%$ humidity), showing minimal degradation in optical performance. Operational failure occurred after environmental aging life testing at $110^{\circ} \mathrm{C}$ for 216 hours. 


\section{Chapter 1. Introduction}

With the increased use of multi-core processors arises the need for dramatic increases in bandwidth to maximize computing system performance. Current copper based circuit technology is becoming a limiting factor in high input/output $(\mathrm{I} / \mathrm{O})$ applications as processors are improving at a faster rate than are developments to increase the number of $\mathrm{I} / \mathrm{O}$ pins (1). Also limiting performance are the high losses associated with resistive and inductive heat capacitance with the use of dense copper circuitry. One solution is to utilize optical waveguide technology to overcome these bandwidth and loss restrictions. The use of this technology virtually eliminates the heat and cross-talk loss seen in copper circuitry, while also operating at a higher bandwidth.

\subsection{Motivation}

The motivation for developing optical waveguide technology as an alternative to conventional copper circuitry is well documented, summarized by its ability to "in principle address most, if not all, of the problems encountered in electrical interconnections" (2). Conventional electronics are largely limited by electromagnetic radiation to which optical interconnects are not susceptible $(3,4)$. Waveguide technology offers high bandwidth $\mathrm{I} / \mathrm{O}$ ( $\mathrm{Tb} / \mathrm{sec}$ for multi-rack systems, $>10 \mathrm{~Gb} / \mathrm{sec}$ for single board applications (5-7)) with a reduction in thermal radiation, and by relation, required cooling capability. These are significant considerations for sophisticated rack systems required for avionics, where space and weight are at a premium.

To capitalize on the advantages of waveguides and optical interconnects, the principal motivation must be a successful merger of optical and electrical circuitry, resulting in a hybrid Optical-Electrical-Printed-Wiring-Board (OEPWB). To achieve this, it is necessary to develop waveguide fabrication techniques compatible with current printed circuit board (PCB) manufacturing practices.

\subsection{Challenges}

The introduction of embedded optical waveguides into printed circuit boards offers significant challenges. The most substantial hurdle is manufacturing cost, as any new technology has to be competitive with current electrical printed circuit board (PCB) products to be sustainable.

Major costs reside in materials and tooling required for the standard approach of lithographic patterning. The standard approach for fabricating waveguides in the laboratory utilizes spin coating of cladding and core materials and photolithographic 
patterning using high resolution, quartz glass masks. Scaling these processes up for use on large substrates $\left(>2500 \mathrm{~cm}^{2}\right)$ poses a significant physical and financial challenge.

A major cost obstacle is the ultra-high resolution required of the mask to ensure high levels of waveguide performance. Surface roughness in the sidewalls of the waveguides leads to signal loss due to light scattering, seen specifically in waveguide bends or turns (8). This sidewall roughness is directly linked with the resolution of the mask used for patterning, and higher resolution printing comes at a significant price increase. This cost increase is magnified by the increase in board size as well. Another drawback to printed mask technology is the inflexibility it provides in a manufacturing setting. Customers with an original board pattern would require a unique mask, at a very high associated cost. Any change in the waveguide pattern then requires a different mask.

Material processing is also a concern, both physically and financially. The standard approach to material deposition is spinning, which produces very uniform layers but results in significant material waste. After UV patterning, most of the deposited material is chemically developed away, resulting in even more material waste. With high waveguide material costs (many thousands of dollars per liter of uncured material), this approach becomes financially prohibitive when working on large substrates and looking towards mass production. Physical challenges are also of concern, as the uncured waveguide material remains tacky until fully cured. This means that contact lithography becomes significantly more complicated as the mask cannot make contact with the uncured material or it will firmly adhere to the substrate upon exposure. The waveguide material also requires the use of organic solvents for development processing which is often undesirable in PCB manufacturing facilities, where environmental and worker safety regulations mandate very controlled handling and disposal methods. After waveguides are successfully fabricated, they must also be able to withstand the general PCB manufacturing environment without suffering damage or contamination.

The most significant hurdle in achieving functional OEPWBs is developing a method of interfacing $\mathrm{I} / \mathrm{O}$ with the optical layer through connectorization of the optical circuits. Techniques are needed for turning the optical signals out of plane, as are methods of passively aligning and securing external cabling. With lateral alignment tolerances in the range of ten micrometers (9), board registration becomes of great importance. Making things more difficult is the transparency of the waveguides, making them difficult to recognize on some vision systems. 


\section{Chapter 2. Literature Review}

\subsection{Fabrication Methods}

\subsubsection{Material Application Methods}

The polymers widely used for the creation of waveguides are very expensive, and much of the material is wasted in the fabrication process. Current processing relies on spin coating, where an excess of uncured material is puddled in the center of a static substrate. Using an excess of material ensures complete sample coverage, reducing the risk of discontinuities in the finished layer. After the liquid material is deposited, the substrate is spun at sufficient angular velocity to build up centrifugal force in the liquid puddle, forcing it outward radially. The layer thickness is determined by the spin speed and the viscosity of the fluid, with faster spin speeds and lower viscosities leading to thinner layers. This process is terribly inefficient, utilizing only $2-5 \%$ of the material dispensed onto the substrate (10) where the remainder is spun off as waste, requiring hazardous material disposal methods. Prior to disposal, this excess material forms droplets which accumulate on the walls of the coating chamber, a potential source of surface contamination (11) which can be detrimental to waveguide integrity. Uniformity in layer thickness suffers due to increased edge beading which arises from a lack of radial symmetry in the rectangle substrates used for PCBs (12). After blanket layers of material are applied to the substrate, only a small fraction of material is actually patterned into waveguides; the excess is developed away as waste which further decreases the material transfer efficiency. Though it produces excellent results, this standard approach is not well suited for producing the large patterns required for "next generation" circuit boards. Spin coating is not a realistic solution when substrates are in excess of $2500 \mathrm{~cm}^{2}$.

Spray coating offers greater layer uniformity with the ability to cover larger substrates and varied topographies, important when working with a fiber-weave material such as FR4 (13-15). This process is also not as susceptible to variance in the separation distance between the spray head and the substrate surface. Drawbacks include the need to dilute materials to develop proper spray conditions, and material waste due to overspray where only $70 \%$ to $80 \%$ of the dispensed material accumulates on the substrate surface (16). The remaining $20 \%$ to $30 \%$ accumulates on equipment, necessitating periodic cleaning for proper operation.

Doctor-blading has proven to be an effective method for depositing uniform layers (17), capable of greatly reducing the amount of wasted material when compared to spinning and spray coating ( $\sim 5 \%$ material loss for fully characterized setup) (18). Doctor-blading is a contact process, where a sharp blade is swept across the substrate surface at a fixed height. Reserve coating material is applied on the lead edge of the blade, and a thin film is left after the blade passes over the substrate. Doctor-blading is capable of applying 
very uniform layers, but is not suited for irregular topographies or isolating regions of the coating material.

Ink-jetting is a technology that has been investigated for the deposition of polymers, as it provides similar benefits with more localized deposition and greater material transfer efficiency (19-21). Material droplets are ejected from a fluid reservoir through a nozzle using a piezoelectric actuator/valve system. Some ink-jet systems are pressurized to allow for continuous pressure based dispensing. The effects of fluid properties on the ink-jetting procedure and resulting structures have been researched (22) and the process has been successfully demonstrated for dispensing lenses and discrete waveguides (23).

Syringe based dispensing offers even greater material transfer efficiency, along with the ability to localize deposition using inexpensive disposable components. This is a pressure-based process which extrudes material through syringe tips which are available in many sizes, cross-sections, and taper geometries. This technology is inexpensive and easy to implement, but requires minimal separation (dispense height) between the dispense tip and the substrate surface ( $<200$ um typically). The compressibility of the air in the fluid reservoir and the changing fluid level over the course of dispensing complicate the process (24-26).

\subsubsection{Patterning Methods}

There are a number of proven methods for patterning polymer waveguides, the most conventional approach being photolithographic exposure. For this approach, a blanket layer of polymer is applied to the substrate surface to receive UV exposure. A mask with the desired pattern is suspended over the uncured polymer, and backlit with UV light. The polymer layer is cured wherever the light is able to pass through the mask, and the remaining material is then chemically developed away. The resolution of the resulting features is dependent on the resolution of the printed mask.

Patterning waveguides by laser ablation has been successfully demonstrated as well (27) (28). This approach relies on using intense laser pulses to ablate unwanted polymer material. Excimer laser, $\mathrm{CO}_{2}$ laser, and Neodymium-doped Yttrium Aluminum Garnet (Nd:YAG) laser technology is currently utilized in the PCB industry for via drilling in high density circuit boards, but published losses for waveguides patterned with $\mathrm{CO}_{2}$ and Nd:YAG lasers are high ( $\sim 10 \mathrm{~dB}$, including propagation and coupling losses) due to increased surface roughness of the sidewalls caused by thermal damage during laser machining $(29,30)$.

Molding and embossing are similar approaches successfully utilized for patterning multimode (3, 17, 31-33) and single mode waveguides (34), as well as complex splitter structures (35). Molding, or "groove-filling", relies on pressing a die with positive features into an intermediate material to create a mold, which can be filled with polymer to create waveguides (Figure 2.1). For embossing, or "rib-cladding", a die with negative 
features (waveguide pattern) is pressed into a layer of polymer, displacing all of the material except in the area of the negative features. When the mold is removed, only waveguides remain. These methods are capable of creating very repeatable structures, but are limited in design flexibility similar to photolithography.

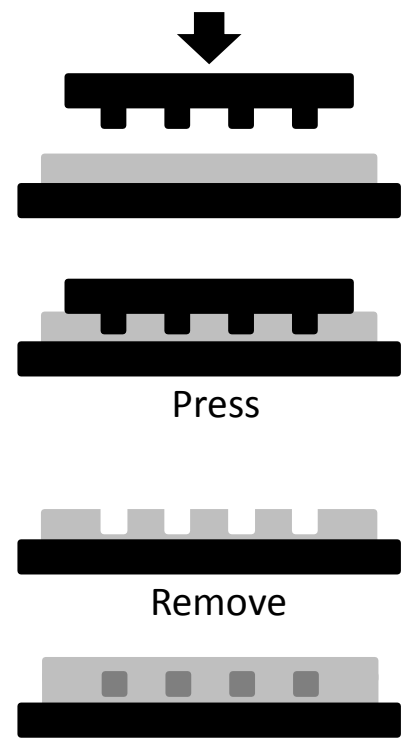

Fill and Clad

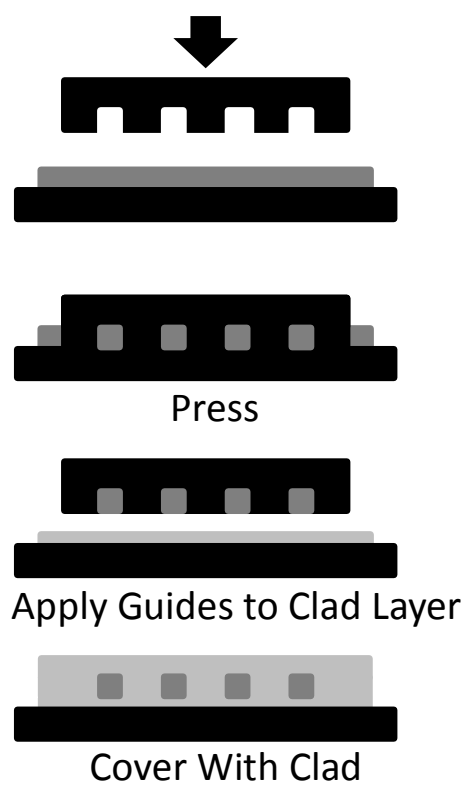

Figure 2.1. Molding, or "groove-filling" method (left) and embossing, or "rib-cladding" method (right) of fabricating waveguides

While syringe dispensing and ink-jetting have been demonstrated for depositing blanket layers of materials, they have also been investigated for their ability to create waveguides which require no additional patterning. These direct write approaches offer very controlled methods of dispensing beads of material which do not require UV patterning or chemical development, eliminating waste material. The major limitation with this approach is the difficulty in achieving an aspect ratio approaching 1:1 for waveguide height and width, while keeping dimensions close to the desired $50 \mu \mathrm{m}$. Multimode waveguides have been ink-jetted with a height to width ratio of 1:5 ( $\sim 20 \mu \mathrm{m}$ tall, $\sim 100$ $\mu \mathrm{m}$ wide) (19), and syringed with a height to width ratio of 1:15 $(\sim 16 \mu \mathrm{m}$ tall, $\sim 250 \mu \mathrm{m}$ wide) in a single pass (36), and 1:6 ( 40 $\mu \mathrm{m}$ tall, $\sim 250 \mu \mathrm{m}$ wide) by stacking multiple passes (37). Ink-jetting is not sensitive to variances in dispense height like syringe dispensing, but it has not demonstrated the ability to match the minimum feature sizes capable with syringe dispensing. Single mode waveguides have been produced by syringe dispensing, which have an aspect ratio of 1:20 ( 0.8 $\mu \mathrm{m}$ tall, $\sim 16 \mu \mathrm{m}$ wide), using a micropipette with an inner diameter of $10 \mu \mathrm{m}$ (38). Ink-jetted and syringe dispensed waveguides are parabolic in shape, and require further improvement to reduce line widths and approach an aspect ratio of 1:1. 
Another direct-write approach to patterning waveguides is laser direct writing, where a focused laser beam is used to expose the uncured waveguide material, eliminating the need for a costly high resolution mask. The ability to laser direct write multimode waveguides which perform comparably to those fabricated by standard photolithography has been demonstrated $(7,39-41)$, and the technology has resulted in waveguides of single mode sizes (4-6 $\mu \mathrm{m})(42)$. Laser direct writing allows for the creation of unique patterns on the fly through the use of CAD design tools, making it very flexible. This is a non-contact process, removing the complications associated with the tackiness of the uncured waveguide material, and allows for patterning on larger substrate sizes $(>2500$ $\left.\mathrm{cm}^{2}\right)$.

\subsubsection{Light Turning Methods}

Essential to solving the challenge of developing a fully functional OEPWB is a method of turning the light signals out-of-the-board plane. Many methods have been investigated, largely centering on physical modification of the waveguide end-faces to create $45^{\circ}$ structures, angled exposure of the waveguide material for the same purpose, or by implantation of external reflector elements.

Physical modification approaches allow the reflector face to be integrated directly into the waveguide structure, reducing the number of components required to turn the signals out of the board plane and thus easing the alignment process. One straightforward approach is using a dicing blade with a $45^{\circ}$ face to dice across the waveguides, creating angled waveguide end-faces (43-45) which can be metalized (Figure 2). A similar approach is to use diamond coated chamfer milling tools to micro-mill across the waveguides, resulting in angled end-faces (17). With these techniques, die masters can be created with machined $45^{\circ}$ end faces for use in the "groove filling" method of molding waveguides $(33,46)$. Waveguide end faces on the edge of a sample can also be cleaved on an angle using a microtome (47-48).

A similar approach to physical modification that does not use tooling to alter the waveguides is the use of angled laser ablation $(36,47,49,50)$. Intense laser pulses with an incident angle of $45^{\circ}$ with respect to the substrate surface ablate the cured polymer waveguides at an angle, creating a rhombus shaped cavity (Figure 2.2). Reactive ion etching (RIE) can also be used to remove material to create angled end-faces $(40,51)$.

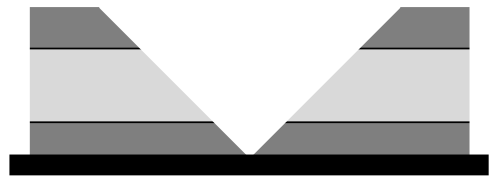

(A)

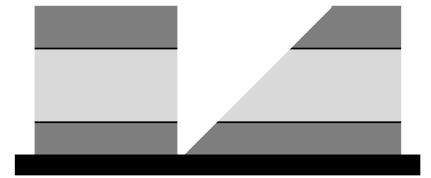

(B)

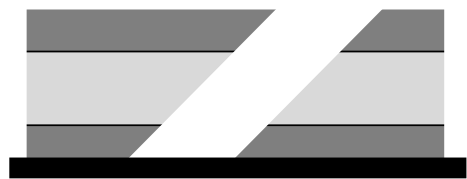

(C)

Figure 2.2. Profiles of waveguide end faces created by way of (A) micro milling, dicing, and reactive ion etching, $(\mathrm{B})$ dicing, and $(\mathrm{C})$ laser ablation. 
Lithographic approaches to creating reflectors have also been successful, including angled UV exposure of uncured polymer. By adjusting the angle between the incident UV light and the substrate surface during exposure, rhombus-shaped features can be patterned which can either serve as total internal reflection (TIR) mirror faces, or they can be metalized to serve as a reflector face for turning the light out of plane. The incident angle of the UV light can be modulated by either adjusting the orientation of the light emission (52-54), or by tilting the substrate beneath standard vertically oriented exposure tools (55).

Another lithographic approach is the use of gray-scale lithography for patterning angled structures (56-57). By varying the density of the metal coating of the lithographic mask, a light gradient can be passed through to the uncured waveguide material. The lower levels of light penetrate less deeply than the higher levels of light, resulting in a sloped feature after the uncured material is developed away.

Imbedding components into the optical layer has been investigated for turning light signals out of the board plane $(5,58,59)$. The fabrication of reflector components can be achieved in many ways, such as etched silicon or machined, metalized glass reflectors (60), injection molded parts, reflectors coupled with microlenses or ball lenses $(58,61)$, or photo-patterned polymer parts, where deep proton writing (DPW) is used to selectively expose and then etch polymer photoresist (PMMA) to create an implantable $45^{\circ}$ coupling structure (62). This process is not suitable for mass production due to the long development time (5 hours), but demonstrates an implantable component suitable for out of plane signal routing (63).

However the light signal is turned out of plane, the components there must have some mechanism for securing external cabling to the board, and alignment between all components must be established. These components must all be successfully coupled together and embedded within the outer board layers with the ability to demonstrate acceptable performances levels for data transmission $(>10 \mathrm{~Gb} / \mathrm{s})$.

\subsection{Current PCB Technology}

To successfully transition printed circuit technology from the electrical to the optical domain, the new product cannot cost more than the existing technology. Utilization of fabrication tools currently utilized by the PCB manufacturing industry provides a way to drive down the costs associated with the fabrication and connectorization of waveguides. Cost of materials must be a consideration when working with such large substrates. With most of the waveguide material being removed as waste in the spin coating process, and the price of waveguide material being high, better solutions for material deposition are required. Discrete deposition of liquid materials is a practice already in place in the PCB industry. One technology currently being embraced by PCB manufacturers is ink-jetting for board legend applications, used to label circuitry (64). This toolset can be used for jetting bands of liquid waveguide polymer only in the areas that are to contain optical 
circuitry, drastically reducing the amount of waste material seen with current methods such as spinning or doctor-blading blanket layers. While this approach can be used to provide narrow bands which can be laser defined, work is being done to explore the possibility of directly jetting finished product waveguides which would eliminate the need for an expensive laser tool as well as the use of organic solvents (19). Another similar approach which could achieve these same benefits is depositing even narrower beads of material with round shape (rather than the semicircular shape obtained from jetting) by a relatively low cost syringe deposition system. Syringe dispenser units are currently used to apply sealants and adhesives locally to boards, providing excellent efficiency in material transfer. Spray coater systems are widely used to apply conformal coatings to PCBs and offer excellent process automation. Each of these coating systems can be closed loop, conveyor based setups, offering excellent throughput. Capable of handling large substrates, these automated systems are capable of self-cleaning, thus improving reliability. These are non-contact approaches which would drastically reduce material costs as well as reduce the role of organic solvents in waveguide fabrication.

While PCB manufacturers make use of standard photolithographic exposure units, the industry is quickly adapting to more versatile methods of UV exposure, such as laser direct imaging, and digital light projection (DLP) based direct imaging tools which allow for the dynamic and high quality creation of layer "artwork" (circuit routing patterns). In the case of laser direct imaging, a focused UV laser source is raster scanned across the substrate to create the desired pattern. This approach is limited by the size of the pixels in the raster scanning process. DLP based direct imaging relies on rectangular arrays of microscopic hinge-mounted mirrors which are illuminated in parallel (65). These mirrors can either direct light towards or away from the substrate as a way of exposing pixels. Because the mirrors are illuminated in parallel, the entire pattern can be exposed at one time, a significant improvement over laser direct imaging, which provides serial exposure of pixels. Direct imaging also allows for gray scale patterning.

\subsection{Background Conclusions}

Direct-write material deposition and patterning offers the clearest path to successful patterning on large substrates, and it also offers the most straightforward transition to commercial manufacturing, as many similar tools are already in place in the PCB industry. A manufacturable method of turning the light signals out of plane must be developed, with some means of aligning and coupling external cabling to the optical channels. To demonstrate the successful creation of OEPWBs, it will be necessary to develop the direct writing (dispensing and exposure) capabilities to achieve reliable and repeatable creation of passive waveguide devices/structures such as straight and curved waveguides, splitters/combiners. It is also necessary to connectorize the optical layer for passive, out of plane coupling using standard multimode fiber cabling. 


\section{Chapter 3. Direct Write Waveguide}

\section{Fabrication Tool}

To accomplish the difficult task of creating an "all-in-one" tool capable of fabricating waveguides on large substrates $\left(>2500 \mathrm{~cm}^{2}\right)$, it was necessary to leverage the existing knowledge base of micro-fabrication techniques available at Michigan Tech.

In creating new tools and processes, building upon standard photolithographic practices currently utilized in the Michigan Tech Microfabrication Facility offered the clearest path to success. The substrate material used for all experiments was FR4, cut into $100 \mathrm{~mm}$ squares. The waveguide material used was Dow Corning's OE4140 (core) and OE4141 (clad) photo-patternable polysiloxane. The basic process steps, illustrated in Figure 3.1, were as follows:

1. Clean the FR4 substrate.

a. Begin with an isopropyl alcohol rinse to remove any contaminants that may lead to poor adhesion or the creation of voids in the waveguide material.

b. Blow Nitrogen or filtered air across sample surface to evaporate any remaining isopropyl alcohol and to remove any particulate from the surface of the substrate that may lead to defects in the optical layer.

2. Apply bottom clad layer.

a. Puddle $20-30 \mathrm{~mL}$ of clad material in the center of the substrate.

b. Spin at $500 \mathrm{rpm}(200 \mathrm{rpm} / \mathrm{sec}$ acceleration) for 55 seconds to achieve a 25 $\mu \mathrm{m}$ thick cladding layer.

c. Optional pre-exposure bake at $125^{\circ} \mathrm{C}$ for 3 minutes, on hotplate or in oven, to drive off toluene for somewhat easier handling.

d. Flood expose with $1.2 \mathrm{~J} / \mathrm{cm}^{2}$.

e. Post-exposure bake at $125^{\circ} \mathrm{C}$ for 3 minutes, on hotplate or in oven, to promote cross-polymerization and complete the cure.

3. Apply core layer

a. Puddle 20-30 mL of core material in the center of the substrate.

b. Spin at $200 \mathrm{rpm}(200 \mathrm{rpm} / \mathrm{sec}$ acceleration $)$ for 70 seconds to achieve a 50 $\mu \mathrm{m}$ thick core layer.

c. Optional pre-exposure bake at $125^{\circ} \mathrm{C}$ for 3 minutes, on hotplate or in oven, to drive off toluene for somewhat easier handling.

d. Methyl isobutyl ketone edge bead removal at $500 \mathrm{rpm}(200 \mathrm{rpm} / \mathrm{sec}$ acceleration) for 10 seconds, if needed. Follow with 500 rpm dry spin for 20 seconds. 
4. Align mask and expose.

a. Pattern Exposure with $0.8-1.2 \mathrm{~J} / \mathrm{cm}^{2}$. Do not contact polymer with mask.

b. Post-exposure bake at $125^{\circ} \mathrm{C}$ for 4 minutes, on hotplate or in oven, to promote cross-polymerization and complete the cure.

5. Develop away waste core material leaving only the desired waveguide structures.

a. Puddle mesitylene on substrate, completely coating the entire surface, and let stand for 2 minutes.

b. 5 second mesitylene rinse at $250 \mathrm{rpm}(200 \mathrm{rpm} / \mathrm{sec}$ acceleration $)$.

c. 5 second isopropyl alcohol rinse at $250 \mathrm{rpm}$ ( $200 \mathrm{rpm} / \mathrm{sec}$ acceleration).

d. 30 second dry spin at $1500 \mathrm{rpm}(500 \mathrm{rpm} / \mathrm{sec}$ acceleration $)$.

6. Apply bottom clad layer.

a. Puddle 20-30 mL of clad material in the center of the substrate.

b. Spin at $500 \mathrm{rpm}(200 \mathrm{rpm} / \mathrm{sec}$ acceleration $)$ for 55 seconds to achieve a 25 $\mu \mathrm{m}$ thick cladding layer.

c. Optional pre-exposure bake at $125^{\circ} \mathrm{C}$ for 3 minutes, on hotplate or in oven, to drive off toluene for somewhat easier handling.

d. Flood expose with $1.2 \mathrm{~J} / \mathrm{cm}^{2}$.

e. Post-exposure bake at $125^{\circ} \mathrm{C}$ for 3 minutes, on hotplate or in oven, to promote cross-polymerization and complete the cure.

7. Final hard bake in oven at $150-160^{\circ} \mathrm{C}$ for 30 minutes to accelerate complete curing (material will cure overnight at room temperature, but there is potential for the degradation of environmental stability of the waveguides without a hard bake step).

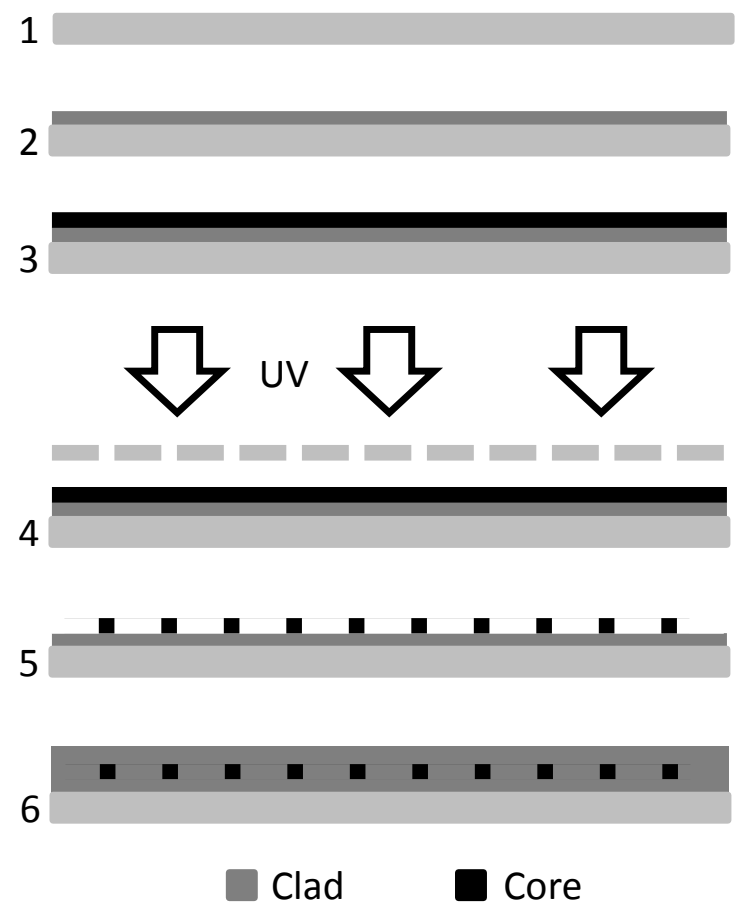

Figure 3.1. General photolithographic processing steps. 
This is the process that served as the standard to compare against for all developed technologies as it consistently produced high quality samples exhibiting losses of 0.04$0.06 \mathrm{~dB} / \mathrm{cm}$ for straight waveguides. Using these established practices of material processing, and existing PCB technologies as a guide, a multi-functional tool was built for the purpose of creating optical waveguide layers in a "direct-write" process, rather than a broad coverage process that has considerable waste and cost.

\subsection{Components}

The development of the direct write fabrication tool follows two thrusts: material deposition through direct dispensing of uncured waveguide material, and photopatterning this material by laser direct writing (curing). The main required components for these two applications are a motion platform to serve as a base for direct write processes, a dispense unit for applying the uncured waveguide material, a UV laser for curing the waveguide material, a sample fixture for securing the substrate flat and in place, and some means of detecting the relative vertical location of the substrate surface and the respective writing tools. The result of integrating these tools with the motion platform (Figure 3.2 and Figure 3.3) is a high resolution writing tool with dynamic patterning capabilities, ideal for research based prototype production.

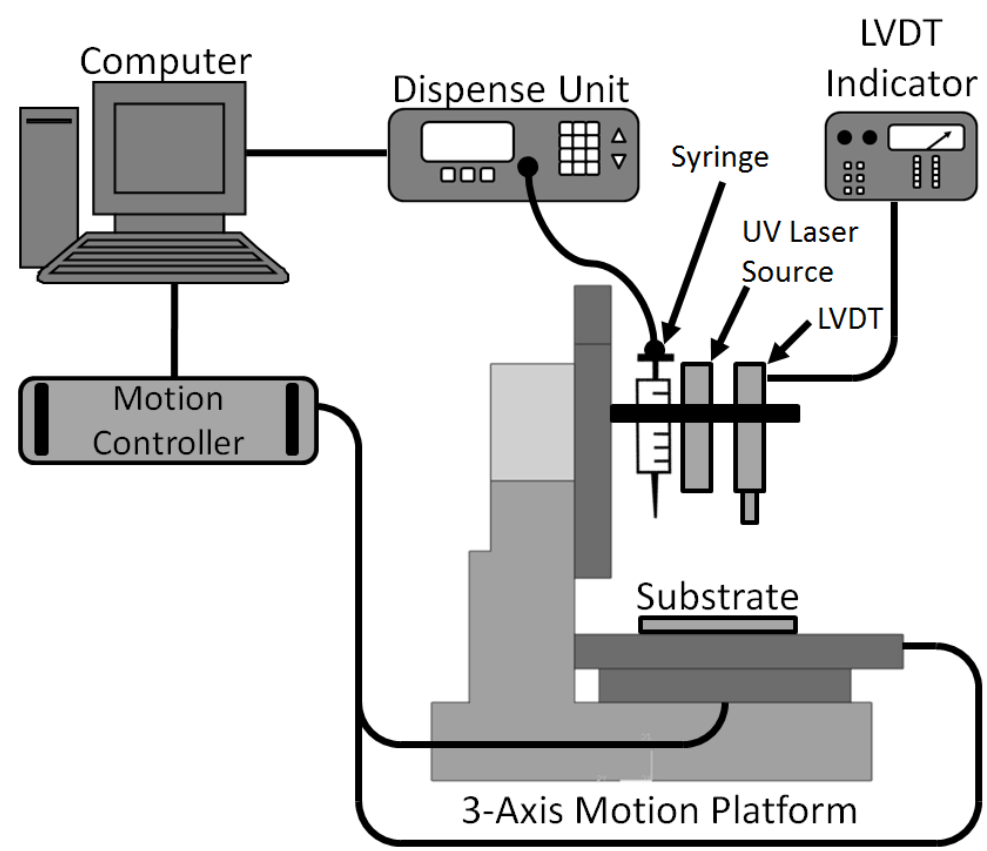

Figure 3.2. Direct write tool schematic. 


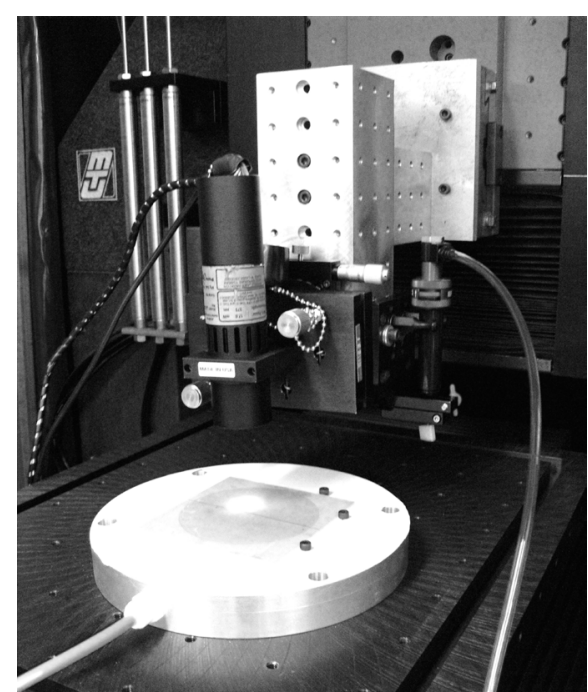

Figure 3.3. Direct write tool platform with mounted laser and syringe dispenser, along with vacuum sample holder.

\subsubsection{Motion Platform}

The base component of the direct write tool is the CNC motion platform used to move the sample with respect to the writing components. This motion platform must be high resolution with excellent repeatability and accuracy, as waveguide performance is largely dependent on reducing the roughness of the sidewalls $(<100 \mathrm{~nm}$ average roughness, Figure 3.4), as well as maintaining smooth transition between features, such as bends and straight sections. The write speeds must be carefully controlled, as they directly affect the energy dose delivered to the uncured waveguide material, which helps determine the final waveguide dimensions. 


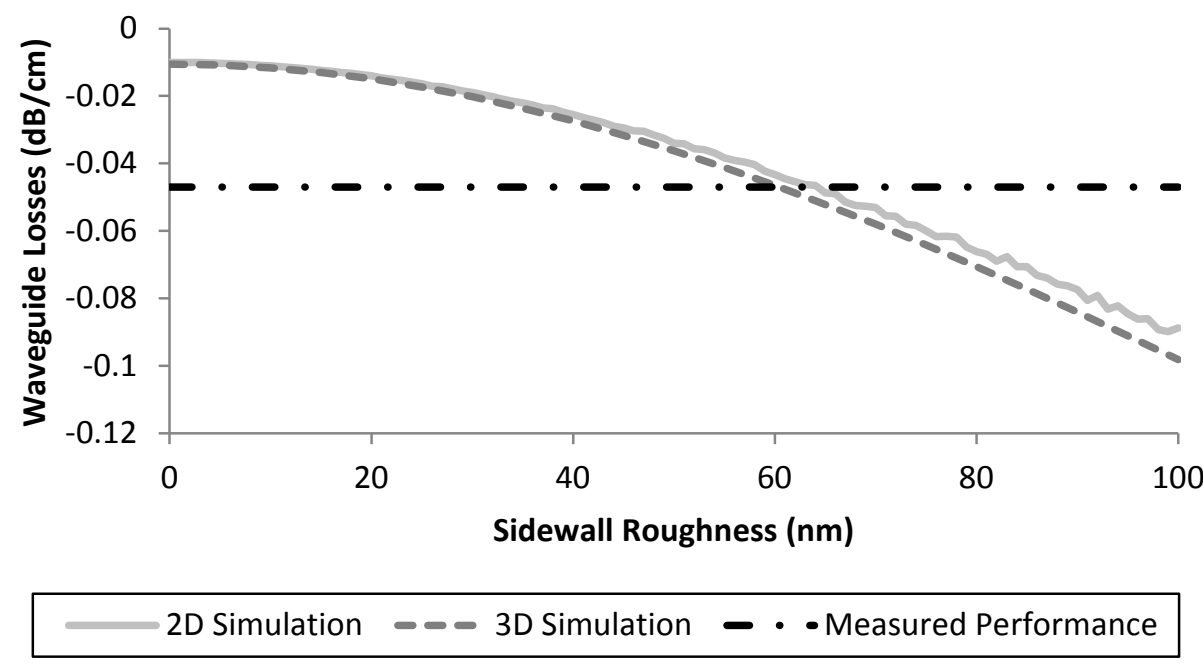

Figure 3.4. The effect of waveguide sidewall roughness on optical performance (Using the 3D Beam Propagation Method to Model the Effects of Lithographic Roughness on the Attenuation of Multimode Polymer Waveguides).

To address these control requirements, an Aerotech ABL1500 air bearing motion platform served as the lateral motion platform of the direct write tool. It features nanometer resolution and is $\mathrm{CNC}$ operated providing 3-D patterning flexibility. With the ability to move at speeds up to $100 \mathrm{~mm} / \mathrm{sec}$, it allows sufficient throughput to be effective for optical-electrical-printed-wiring-board prototype manufacturing. The motion platform offers $400 \mathrm{~mm}$ of travel in the $\mathrm{X}$ and $\mathrm{Y}$-axis

\subsubsection{UV Laser Source}

To pattern waveguides of the desired shape and size, a well contained UV source with a high energy density output is needed, making a laser source a good choice. The emitted wavelength must closely match the absorbance peaks of the liquid waveguide material to ensure effective curing (Figure 3.5). The UV source must be stable in its output, and a low beam divergence is desirable, as will be discussed in Section 4.1.1.

Mounted to the Z-axis of this motion platform is an IQ $\mu 1 \mathrm{C}$ laser system by Power Technology Inc. The IQ $\mu 1 \mathrm{C}$ is a self-contained laser module equipped with a diode laser emitting a $20 \mathrm{~mW}$ continuous wave, elliptical beam at a wavelength of $375 \mathrm{~nm}$. This module is thermoelectrically cooled, and includes "microlensing", which circularizes the elliptical beam output of the diode without the use of correcting prisms. Also included is an adjustable aspheric lens for focusing/collimating the output beam. Using a compact laser diode module significantly reduces the size constraints and associated costs when compared to higher powered gas laser sources. 


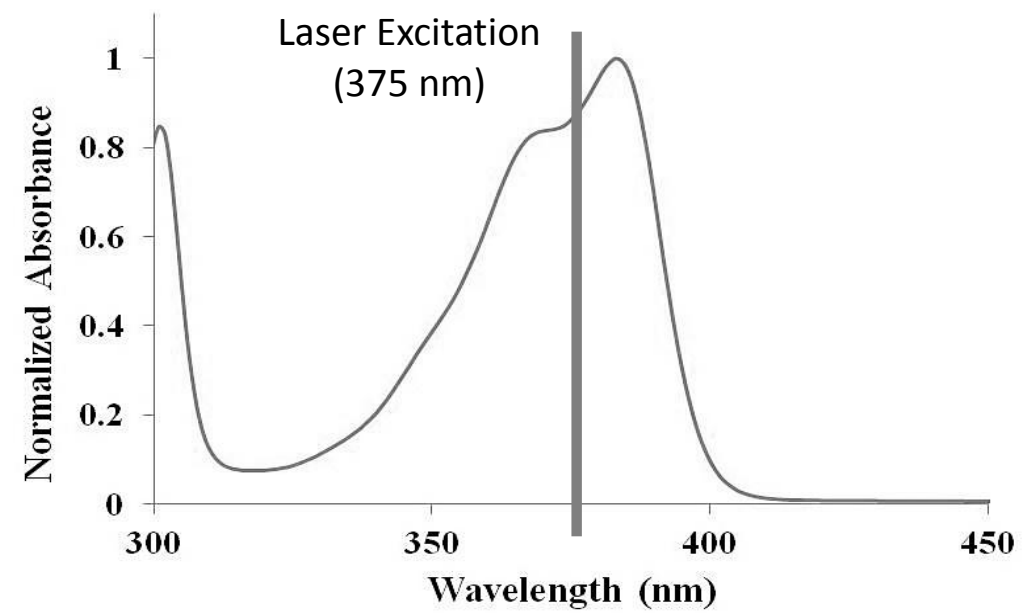

Figure 3.5. UV-Vis absorbance spectrum of Dow Corning OE4100 waveguide material (reproduced with permission from Dow Corning which can be found in Appendix B). 


\subsubsection{Material Dispense Unit}

A method of dispensing uncured waveguide material onto the substrate, both in blanket layers and discrete regions, is required. It is important that this apparatus is able to dispense high viscosity materials (200-1200 cps for Dow Corning OE4100 waveguide materials) that contain organic solvents (toluene). OE4140 and OE4141 are also very tacky materials, so the components to be used must be disposable or easily cleaned after each use.

To meet these needs, mounted to the Z-axis is an Ultimus ${ }^{\mathrm{TM}}$ V High Precision Dispenser and Optimeter ${ }^{\mathrm{TM}}$ by Nordson EFD. The Ultimus ${ }^{\mathrm{TM}} \mathrm{V}$ is a pressure-based unit which utilizes Luer-lock style syringe tips for dispensing liquid materials, and features electronic control of dispense time, air pressure, and vacuum. Syringe tips and material reservoir barrels are able to withstand pressures up to 100 PSI, block UV light, and are disposable.

\subsubsection{Height Detection}

Accurately controlling the vertical position of the dispense tool and the laser source is important for repeatable results. Also important is some means of referencing the vertical position of these tools to the FR4 substrate surface. For laser writing (curing), the vertical position of the uncured material within the beam path will directly affect the waveguide shape and dimensions, due to changes in the cross-sectional shape of the beam along the vertical axis. Vertical positioning is also critical while dispensing uncured waveguide material, as small variances $(\sim 10 \mu \mathrm{m})$ in spacing can result in discontinuous or misshapen waveguide structures.

The method used for obtaining the vertical reference during characterization experiments was physical contact. In the case of direct dispensing, for example, the syringe assembly was loosely affixed in its mount by inserting the syringe tip and reservoir barrel with the clamp screw left untightened This left the assembly with freedom of movement in the vertical direction (Figure 3.6). The vertical axis was slowly lowered until the syringe tip made contact with the substrate surface. The clamp screw was then tightened and the vertical position of the axis was recorded from the $\mathrm{CNC}$ interface to serve as a reference height. By raising the vertical axis by known amounts, the standoff distance of the syringe tip with respect to the substrate surface can be controlled. 


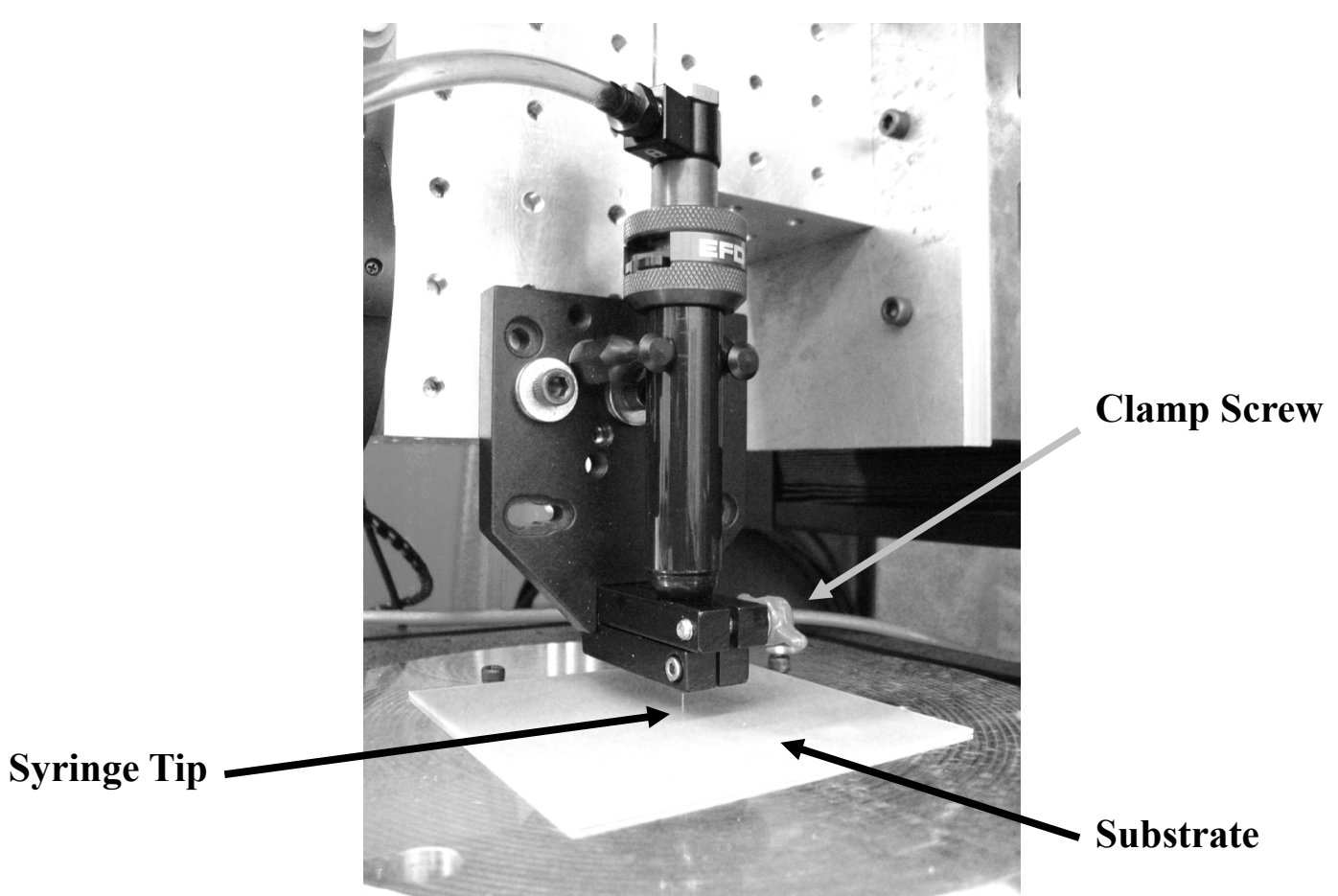

Figure 3.6. Syringe assembly is loosely mounted in fixture to allow safe contact with the substrate surface.

\subsubsection{Sample Fixture}

Securing the substrate in the X-Y plane, while holding it in a position orthogonal to the writing tools, is required for repeatable and reliable fabrication. To achieve this, an aluminum work-plate was fabricated with integrated vacuum channels which were zoned to accommodate different substrate sizes. Using vacuum to hold the sample in place is advantageous as it does not require any clamps or brackets to contact the substrate surface where they might contaminate or disturb uncured waveguide material. This method also provides uniform clamping force, minimizing deflection in the substrate. 


\section{Chapter 4. Laser Direct Write Photo-}

\section{patterning}

Laser direct writing offers the ability to dynamically pattern high quality waveguides on a range of substrate sizes. It is a non-contact process, mitigating the risks involved using a tacky waveguide material. Waveguide patterns can be modified or changed between each sample, allowing for system flexibility with the ability to write at speeds up to 100 $\mathrm{mm} / \mathrm{sec}$, it offers sufficient throughput for the emerging technology of OEPWBs.

\subsection{Basic Writing Parameters}

Initial efforts were focused on characterizing the laser direct write process to determine the optimum writing parameters for creating $50 \mu \mathrm{m}$ square waveguides with optical quality comparable to lithographically patterned waveguides. Critical writing parameters were determined to be the vertical standoff distance of the laser from the substrate, write speed/feed rate (energy dose delivered to the uncured waveguide material), and laser output power (power density, or irradiance).

Standoff distance has a direct effect on the size of the focused laser spot and the final dimensions of the written waveguides (Figure 4.1). If the uncured waveguide is located within the waist of the beam, the resulting waveguides should have more vertical sidewalls than if located above or below the beam waist due to convergence/divergence angles. Also, the waist of the focused beam has the smallest lateral cross section, giving the highest power density. Writing with areas of the beam outside of this waist region will result in larger waveguide widths and lower energy dose amounts for a given power setting and write speed.

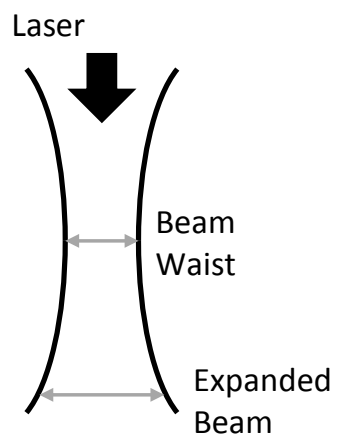

Figure 4.1. Standoff distance defines the location of sample within the beam path, which influences pot size. Writing at the beam waist provides minimum lateral dimensions and maximum dose per time. 
The laser energy dose is controlled by the write speed of the laser in the X-Y plane during the writing process for a fixed laser output power. Because the energy transfer from the laser to the waveguide material is a time integrated process, the length of time that the laser stays in a given spot impacts the amount of energy delivered to the uncured material, affecting cure penetration and overall strength. Dosing is an additive process (Figure 4.2), so modulating the write speeds can also increase or decrease the role that the "tails" of the Gaussian power distribution play in the writing process. By allowing the beam to travel slowly over the uncured material, the lower intensity regions on the perimeter of the spot are able to initiate curing. Conversely, by increasing the feed rates (decreasing the dose), the features reduced in size because only the peak of the Gaussian was able to initiate the curing process. The energy dose amount therefore can impact both waveguide size, and the ability of the waveguide material to withstand development and washes. Irregular curing could impact both the optical properties of the waveguides, as well as the physical dimensions of these structures.

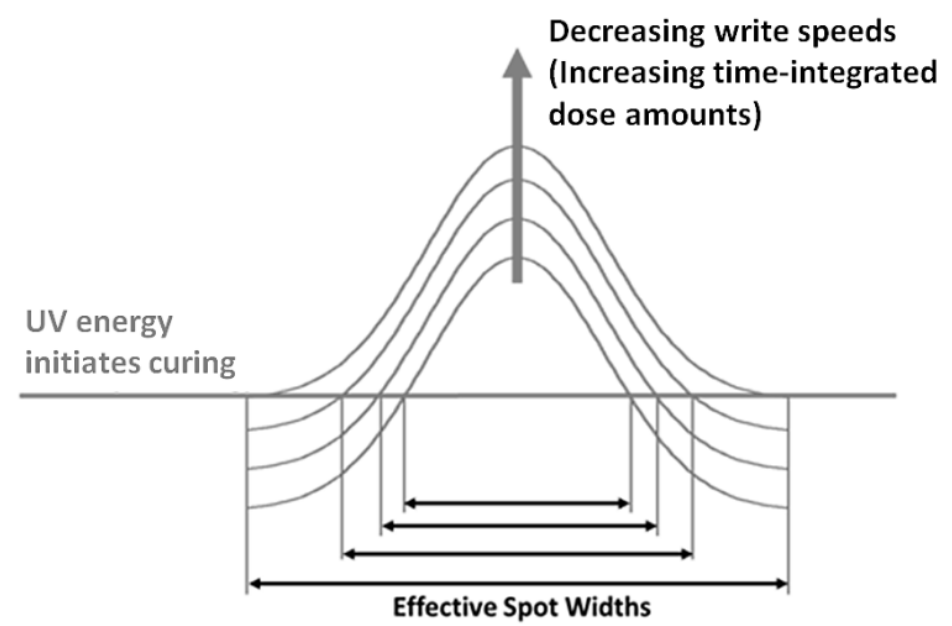

Figure 4.2. Effect of Gaussian power distribution on waveguide width. Slower write speeds lead to larger features.

Write speeds for the different dose amounts were calculated based on approximations of the power density (beam waist diameter assumed to be $50 \mu \mathrm{m}$ ) of the output laser beam and the desired dose value, according to the following equations:

$$
\begin{aligned}
\text { Power Density } & =\frac{\text { Laser Output Power }}{0.25 * \pi *(\text { Beam Diameter })^{2}} \\
\text { Exposure Time } & =\frac{\text { Energy Dose Amount }}{\text { Power Density }} \\
\text { Write Speed } & =\frac{\text { Beam Diameter }}{\text { Exposure Time }}
\end{aligned}
$$


Laser power affects throughput, as writing with a higher power density allows for faster write speeds for a given dose amount. Writing with high peak irradiance allows for deeper penetration of the UV energy through the uncured material thickness. Studies have shown that for a constant energy dosage, cures are stronger and polymerization is more complete with high irradiance cures compared to longer, lower irradiance cures (6667). This results in better adhesion of the cured structures during the development process. It is also important to note that modulating the peak irradiance of the beam can affect the effective beam size, or the portion of the beam that is able to initiate curing (Figure 4.3)

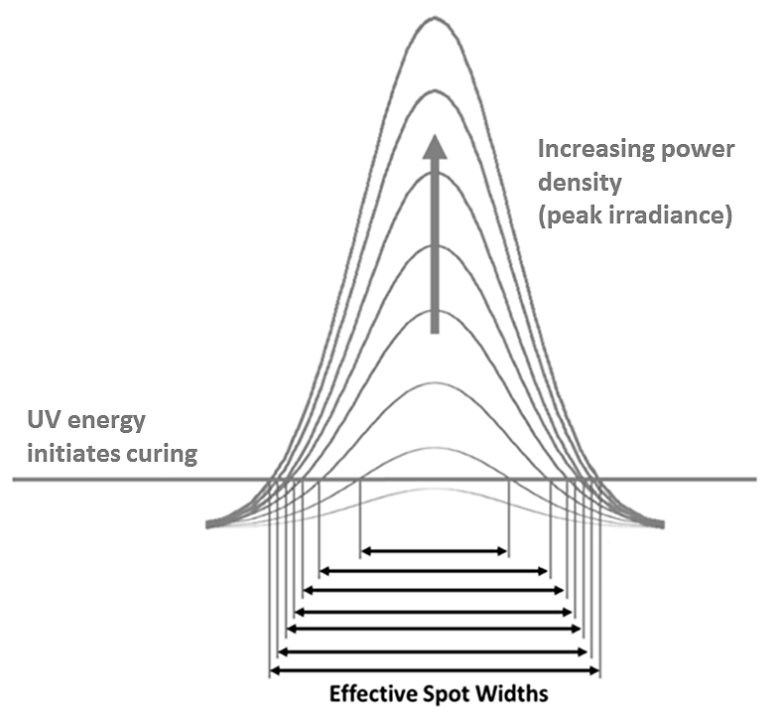

Figure 4.3. Effect of increasing beam irradiance on curing process.

To fully understand the effects of these parameters and to properly characterize the laser direct write process, the following questions were addressed:

1) What is the acceptable standoff distance range for writing waveguides $50 \mu \mathrm{m}$ wide?

2) How does the energy dose amount (write speed) affect the size/shape of the waveguides? What are the effects of over-exposing versus under-exposing?

3) How does the laser output power (power density) affect the size/shape of the waveguides?

\subsubsection{Standoff Distance}

Because standoff distance, write speed, and power density each influence waveguide size, it was important not only to isolate these variables, but to examine them in different combinations to determine their interactions. To characterize the effects of standoff distance and write speed, an array of waveguides were written on a single substrate at a constant laser power. Waveguides were written at six different write speeds (energy doses) for each of five different standoff distances, resulting in 30 individual parameter 
combinations (Figure 4.4). This exercise tested the ability of the direct write tool to create a diverse set of waveguide sizes. The six energy doses chosen were $200 \%, 150 \%$, $125 \%, 100 \%, 75 \%$, and $50 \%$ of the manufacturer recommended dose of $1200 \mathrm{~mJ} / \mathrm{cm}^{2}$ for the waveguide material and the corresponding write speeds are listed in Table 4.1.

Table 4.1

Write speeds for energy doses at a fixed laser output power of $17 \mathrm{~mW}$.

\begin{tabular}{|c|c|c|}
\hline Dose & Dose & Write Speed \\
\hline$(\%)$ & $\left(\mathrm{mJ} / \mathrm{cm}^{2}\right)$ & $(\mathrm{mm} / \mathrm{sec})$ \\
\hline 200 & 2400 & 18.0 \\
150 & 1800 & 24.1 \\
125 & 1500 & 28.9 \\
100 & 1200 & 36.1 \\
75 & 900 & 48.1 \\
50 & 600 & 72.2 \\
\hline
\end{tabular}

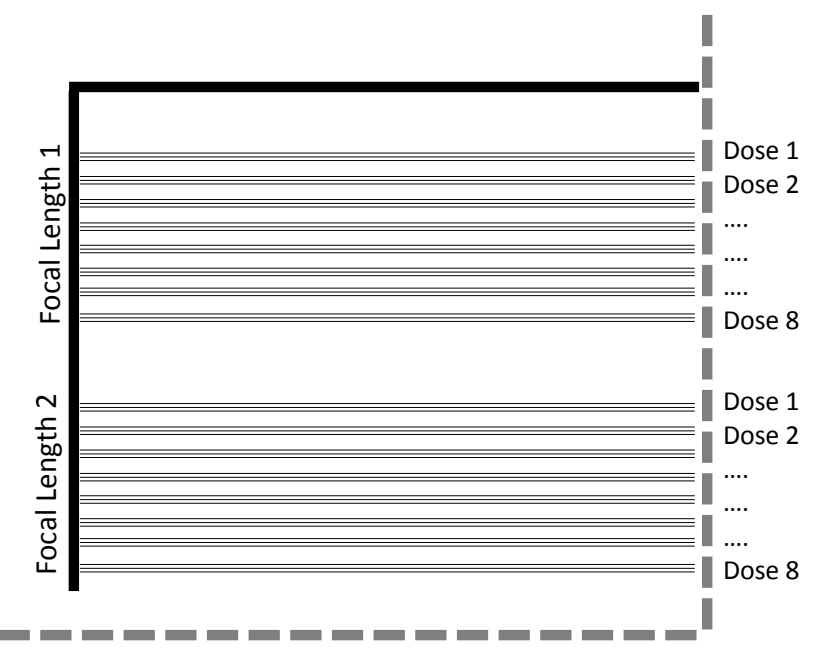

Figure 4.4. Layout of parameter array for direct write sample.

To identify the location of the beam waist (where the narrowest waveguides can be written), beam scans were taken for a standoff distance range of 37 to $50 \mathrm{~mm}$. These scans revealed that the beam waist fell between $45-47 \mathrm{~mm}$ from the laser source (Figure 4.5). The beam scans also revealed ring patterns in the beam cross section, resulting from the focusing optics in the laser module. The aberrations in the beam are undesirable as they can result in non-uniform curing of the waveguide material. The beam was also seen to be slightly elliptical, so writing was repeated in both of the beam axes for comparison. 

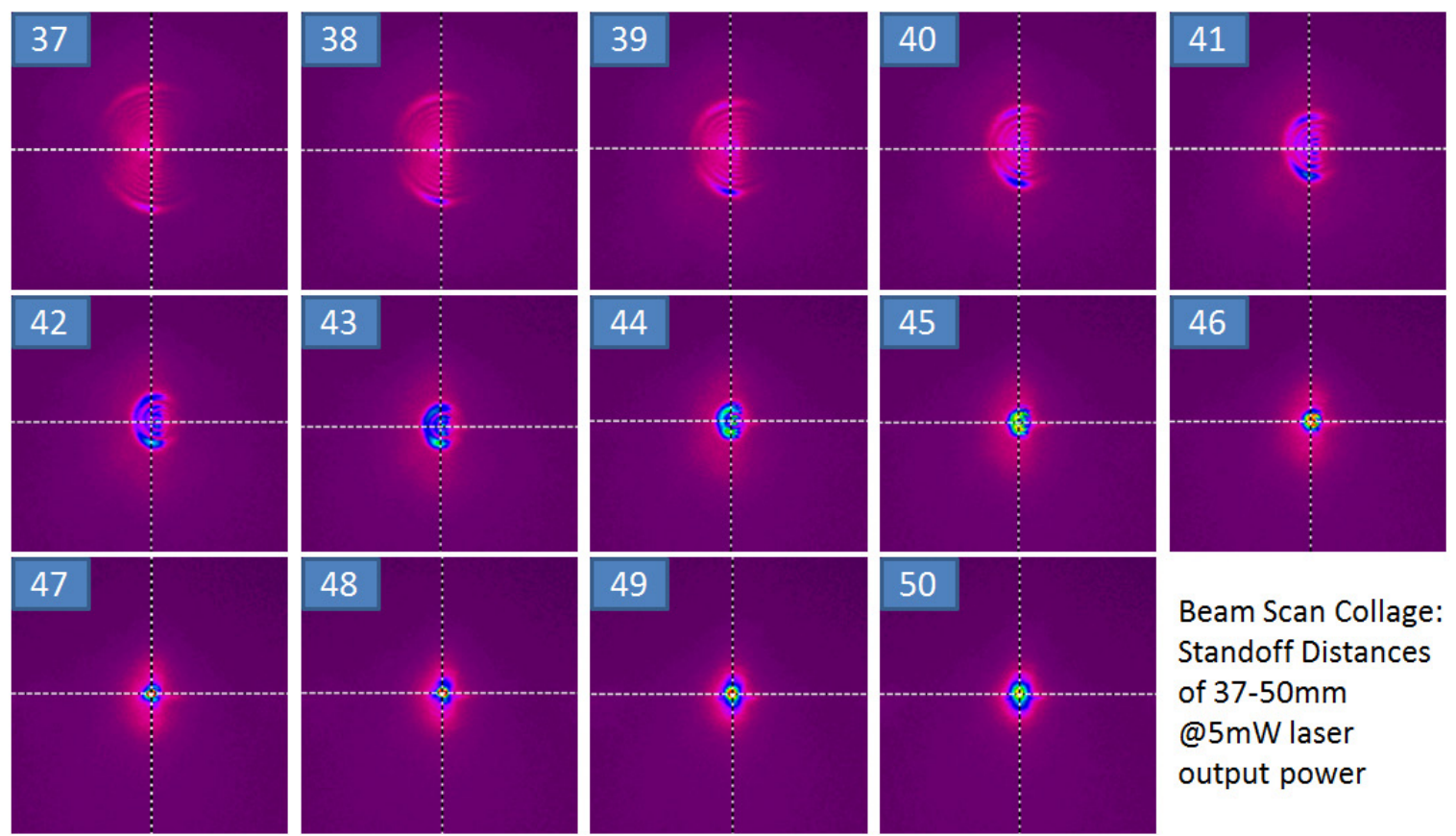

Beam Scan Collage: Standoff Distances of $37-50 \mathrm{~mm}$ $@ 5 \mathrm{~mW}$ laser output power

Figure 4.5. Beam scan collage captured for $5 \mathrm{~mW}$ laser output power depicts beam cross sections at standoff distances of $37-50 \mathrm{~mm}$. Scans are used to identify the standoff distance of the beam waist, which falls between 46-48mm.

The experiment was repeated twice to produce data for a wide range of standoff distances (38-47 $\mathrm{mm})$ and an important result was the verification of the location of the beam waist. Figure 4.6 shows cross sectional views of the waveguides for a single write speed of $36.1 \mathrm{~mm} / \mathrm{sec}$ over the range of standoff distances examined. It is easily seen that the smallest beam size fell at a standoff distance of $45 \mathrm{~mm}$, which matches what was observed in the scans initially collected (Figure 4.5). Figure 4.7 shows how the beam waist falls in the same general region for each of the six doses, and that the standoff distance has a high tolerance compared to the resolution of the vertical axis. This vertical tolerance provides stability in the writing process, helping to ensure dimensional consistency in the fabricated waveguides. 

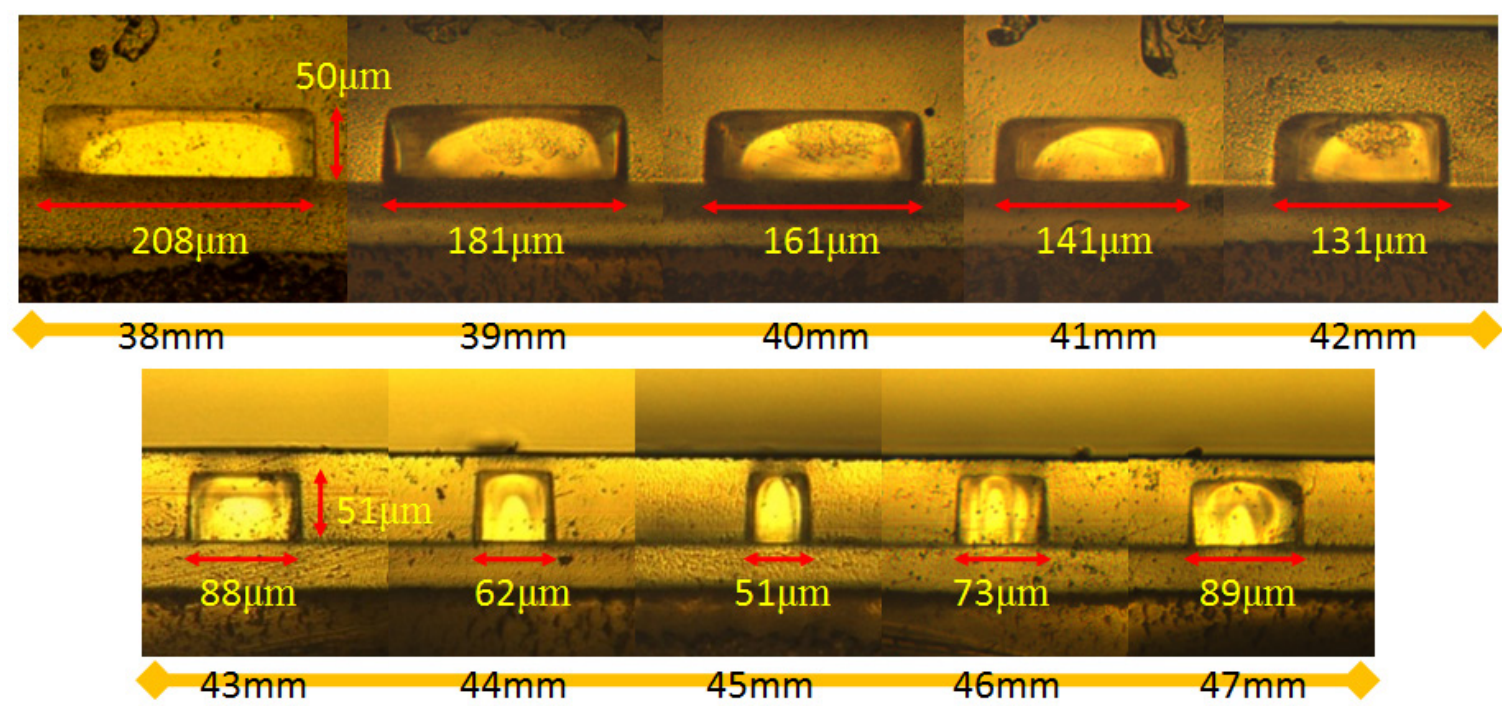

Figure 4.6. Cross sectional views of waveguides showing effect of standoff distance (at recommended dose of $1200 \mathrm{~mJ} / \mathrm{cm}^{2}$ )

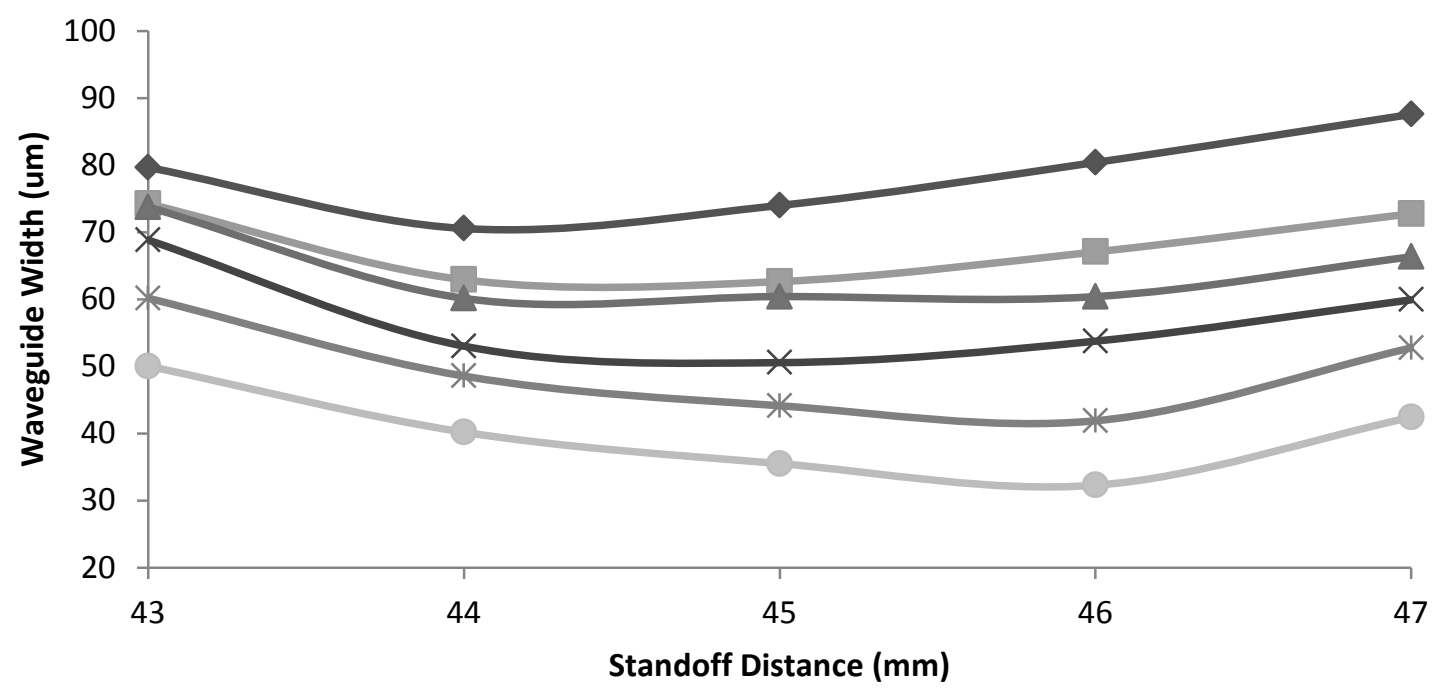

$\multimap 2400 \mathrm{~mJ} / \mathrm{cm}^{\wedge} 2-1800 \mathrm{~mJ} / \mathrm{cm}^{\wedge} 2-1500 \mathrm{~mJ} / \mathrm{cm}^{\wedge} 2$
$\leftarrow 1200 \mathrm{~mJ} / \mathrm{cm}^{\wedge} 2 \multimap 900 \mathrm{~mJ} / \mathrm{cm}^{\wedge} 2-600 \mathrm{~mJ} / \mathrm{cm}^{\wedge} 2$

Figure 4.7. Relationship between standoff distance and resulting waveguide width for each of the six dose amounts examined (manufacturer recommended dose is 1200 $\mathrm{mJ} / \mathrm{cm}^{2}$ ). 


\subsubsection{Write Speed (Energy Dose)}

The results of this study also illustrated that there is a linear relationship between write speed and resulting waveguide width, regardless of standoff distance (Figure 4.8). Having a predictable relationship between dose and waveguide size makes it easy to predict results when designing waveguide systems. It is also notable that doses as low as half the recommended value of $1200 \mathrm{~mJ} / \mathrm{cm}^{2}$ were able to successfully cure the waveguide material, and the resulting waveguides survived development. The results confirm that write speed dictates the role that the tails of the Gaussian power distribution play, as waveguide widths often doubled when going from the fastest write speed (lowest dose) to the slowest (highest dose).

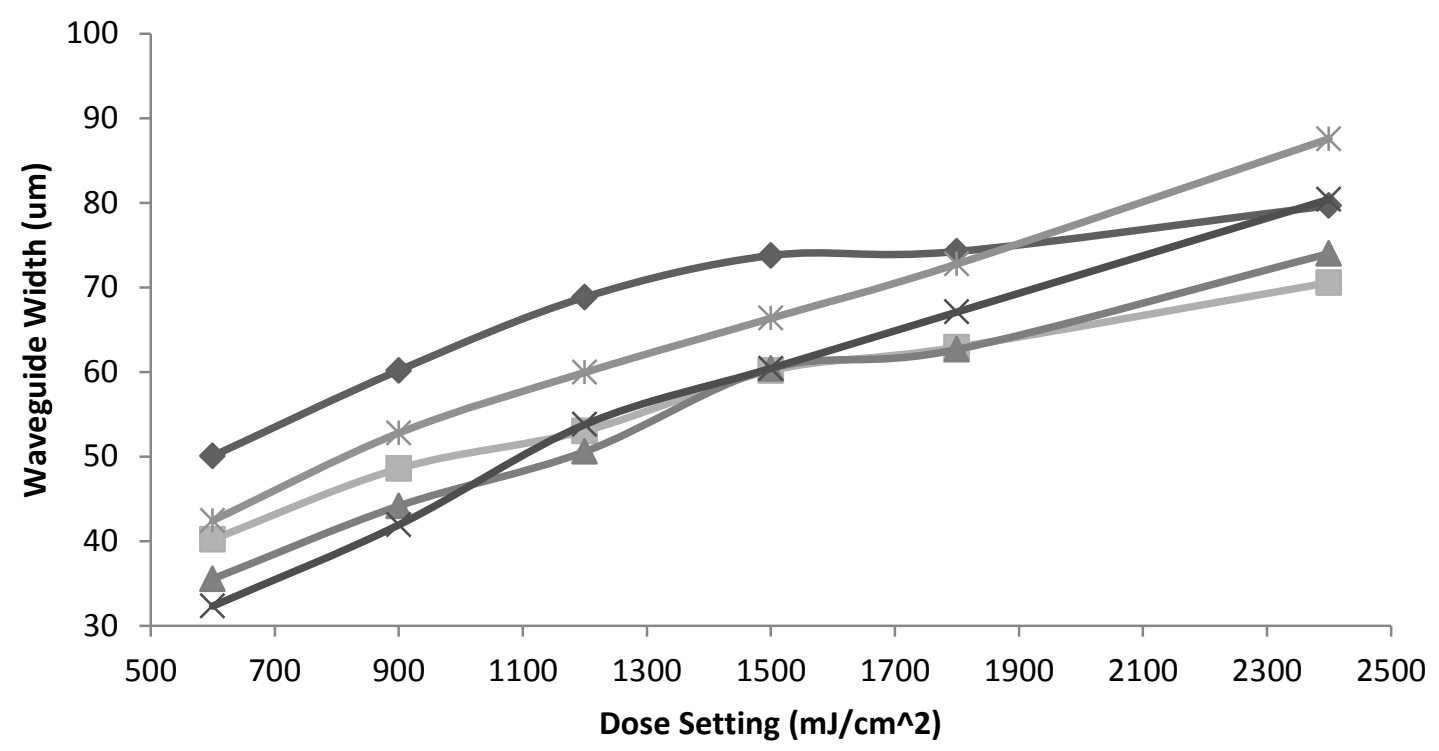

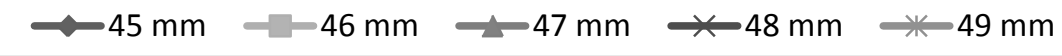

Figure 4.8. Relationship between energy dose amount and resulting waveguide width for each of the five standoff distances.

In summary, a range of doses and standoff distances yielded waveguides from $200 \mu \mathrm{m}$ wide down to $25 \mu \mathrm{m}$, allowing for functionality and versatility within the LDW process. Parameter settings for writing these guides were cataloged to create a calibrated writing process (Appendix A). Table 4.2 details specific writing parameters for fabricating 50 $\mu \mathrm{m}$ wide waveguides 
Table 4.2

Writing parameters for patterning $50 \mu \mathrm{m}$ wide waveguides.

\begin{tabular}{|c|c|c|c|c|}
\hline \multicolumn{2}{|c|}{ Setting } & Width (Axis 1) & Width (Axis 2) & Axis Comparison \\
\hline $\begin{array}{c}\text { Standoff Distance } \\
(\mathrm{mm})\end{array}$ & $\begin{array}{c}\text { Dose } \\
\left(\mathrm{mJ} / \mathrm{cm}^{2}\right)\end{array}$ & $\begin{array}{c}\text { AVG } \\
(\mu \mathrm{m})\end{array}$ & $\begin{array}{c}\text { AVG } \\
(\mu \mathrm{m})\end{array}$ & $\begin{array}{c}\text { Size Difference } \\
(\%)\end{array}$ \\
\hline 45 & 1200 & 50.6 & 49.8 & 1.5 \\
46 & 1200 & 53.8 & 51.8 & 3.8 \\
47 & 900 & 52.8 & 51.5 & 2.4 \\
\hline
\end{tabular}

Waveguides that were $50 \mu \mathrm{m}$ wide were able to be patterned at standoff distances of 43 $47 \mathrm{~mm}$ by adjusting the write speed. In general, each beam axis behaved similarly, where larger doses produce larger guides and vice versa, which can be seen in Figure 4.9. The optical quality of these straight waveguides was determined to be comparable to those produced through standard lithographic methods. However, due to the slightly elliptical beam shape, a difference in waveguide width was observed when writing along one beam axis versus the other (Figure 4.9). This is a problem when writing complex waveguide shapes such as turns, bends, or spirals.
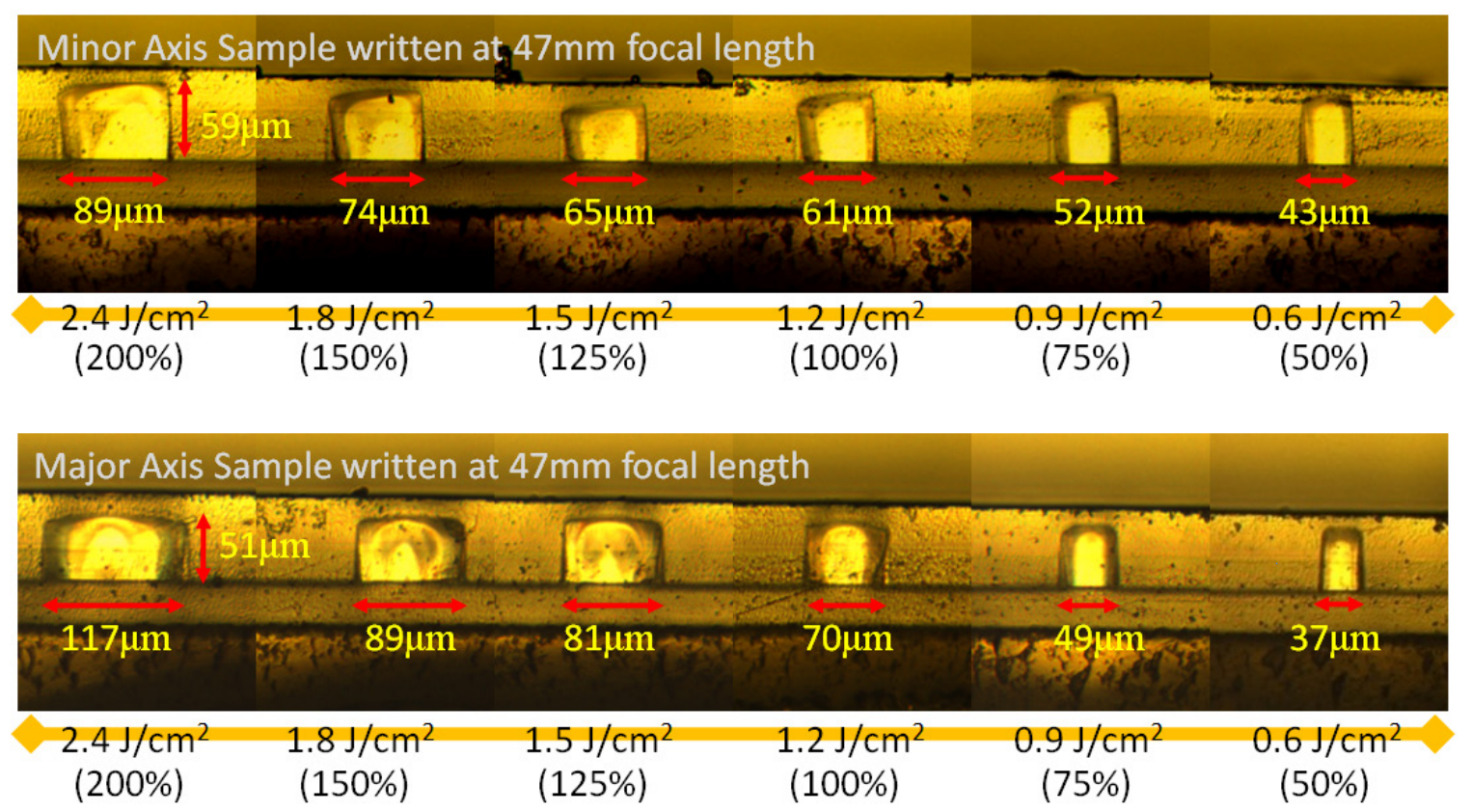

Figure 4.9. Cross sectional views of waveguides written along the major and minor axis of the slightly elliptical beam. The observed trends are the same for each axis, but the specific waveguide dimensions are not uniform between axes. 


\subsubsection{Beam Irradiance}

A second experiment was conducted where waveguides were written at a fixed dose and standoff distance to determine how changes in beam irradiance (Figure 4.3) might impact the size and optical performance of the resulting waveguides. The beam irradiance was modulated by adjusting the laser output power to five different percentage values of the maximum laser output power $(17 \mathrm{~mW})$, and the resulting beam scans at these conditions are seen in Figure 4.10. The dose amount was held constant at $1200 \mathrm{~mJ} / \mathrm{cm}^{2}$ by adjusting the write speed at each power density setting during the writing process. The target power settings were $100 \%, 80 \%, 60 \%, 40 \%$, and $20 \%$ of $17 \mathrm{~mW}$. The correlating output powers and power densities for these settings are listed in Table 4.3, calculated using Equation 4.1 with an assumed spot diameter of $50 \mu \mathrm{m}$.

Table 4.3

Output power and power density values based on percentage of maximum output power $(17 \mathrm{~mW})$.

\begin{tabular}{|c|c|c|c|c|}
\hline Power Setting & Output Power & Power Density & Dose & Write Speed \\
\hline$(\%)$ & $(\mathrm{mW})$ & $\left(\mathrm{W} / \mathrm{cm}^{2}\right)$ & $\left(\mathrm{mJ} / \mathrm{cm}^{2}\right)$ & $(\mathrm{mm} / \mathrm{sec})$ \\
\hline 100 & 17.0 & 866 & 1200 & 36.1 \\
80 & 13.6 & 692 & 1200 & 28.9 \\
60 & 10.2 & 519 & 1200 & 21.6 \\
40 & 6.8 & 346 & 1200 & 14.4 \\
20 & 3.4 & 173 & 1200 & 7.2 \\
\hline
\end{tabular}

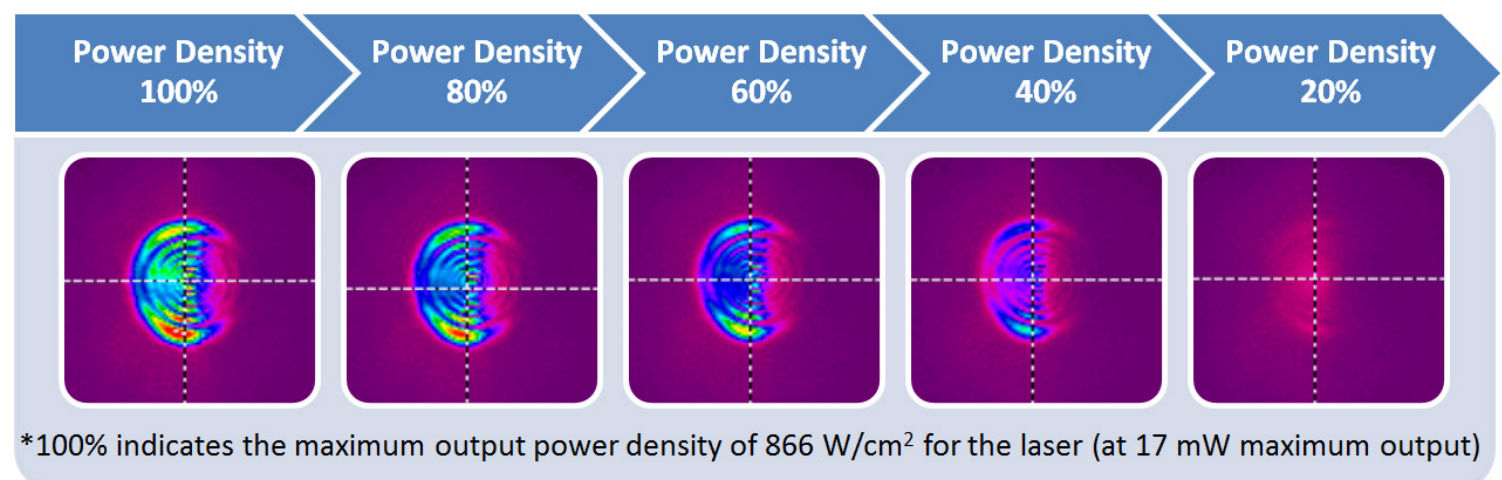

Figure 4.10. Laser output power density characterization experiment setup. Samples were written with a constant energy dose amount $\left(100 \%\right.$ or $\left.1200 \mathrm{~mJ} / \mathrm{cm}^{2}\right)$.

Results show that output beam irradiance has a minor impact in waveguide size, except at the lowest power setting of $3.4 \mathrm{~mW}$, where there was a sharp decrease in waveguide width, as seen in Figure 4.11 and Figure 4.12. This reveals a stable writing process with respect to laser power over nearly all power levels for a constant dose, while still allowing room for fine tuning of waveguide dimensions. This experiment also illustrates 
that very expensive, high power UV sources are not required to successfully pattern multimode waveguides. One important discovery to note is that the waveguides written at the lowest power density of $3.4 \mathrm{~mW}$ did not always withstand the standard developing process. This was likely due to under-exposing the material due to the inability of the UV source to penetrate through the $50 \mu \mathrm{m}$ thick waveguide layer. The result of this is an incomplete cure, which allows the siloxane to be developed away.

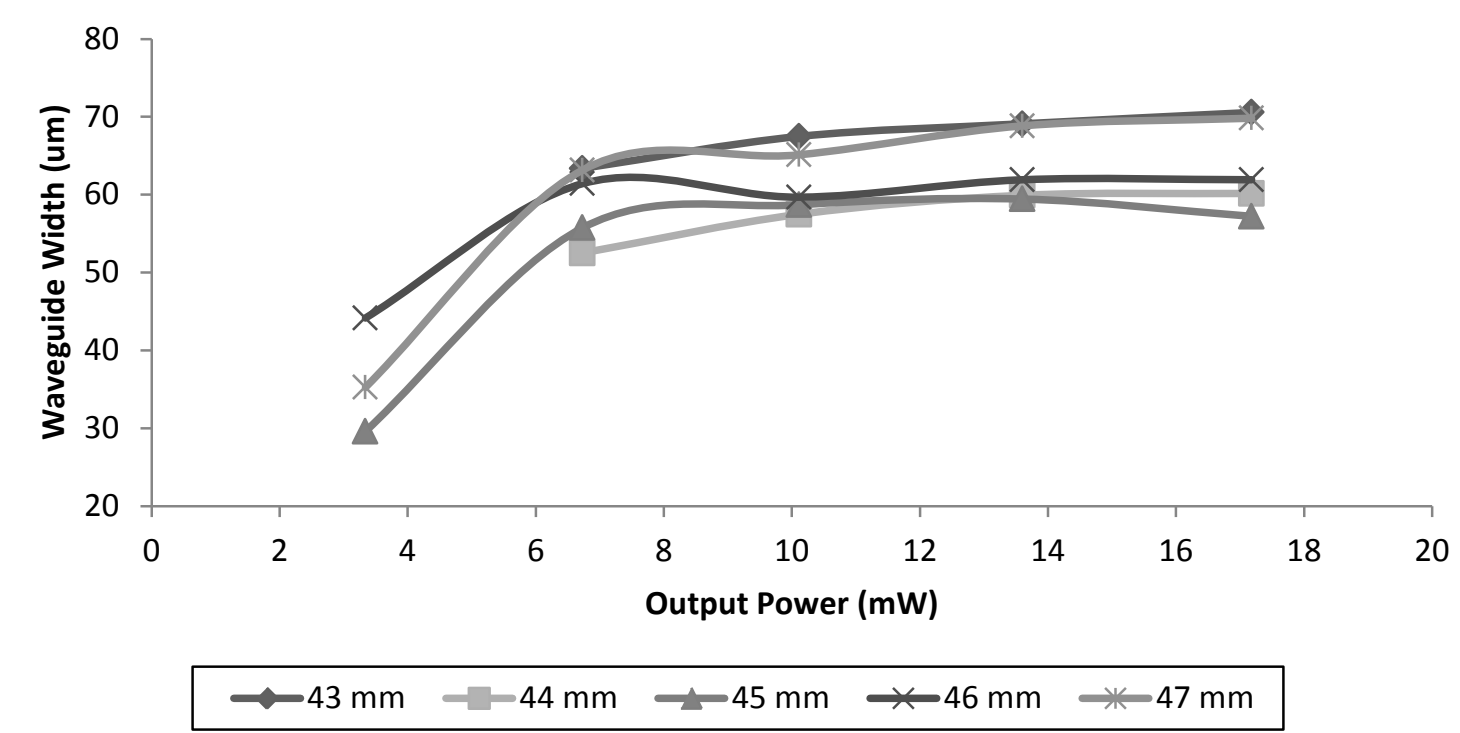

Figure 4.11. Relationship between laser output power and resulting waveguide width for each of the standoff distances for a fixed energy dose amount. Less than $10 \mu \mathrm{m}$ deviation between guide widths over a power range from $7-17 \mathrm{~mW}$.

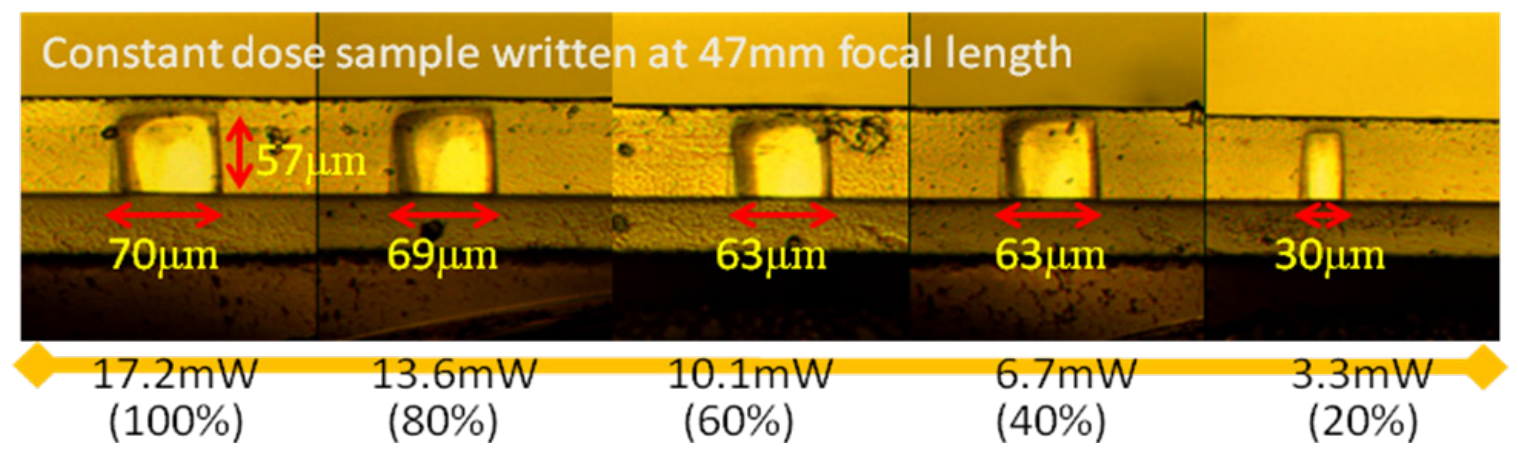

Figure 4.12. Cross sectional views of waveguides showing effect of output power density of laser.

Taking a closer look at what the cause of the drop-off in curing ability at the lowest power setting might be, the output power reading of the GUI software program interface of the laser was verified using an external power meter (1918-C Optical Power Meter and 818 Series Photodetector by Newport). It was observed that the output power displayed 
on the GUI interface was greater than the actual power exiting the laser module (Figure $4.13)$ and the discrepancy was greatest at the low power values $(<5 \mathrm{~mW})$. An output value of $3.4 \mathrm{~mW}$ on the GUI interface corresponded to an actual value of $0.7 \mathrm{~mW}$, which is a power density reduction of $80 \%$. This means that waveguides were being patterned with only $20 \%$ the recommended dose which is the cause for the dramatic decrease in waveguide width and ability to survive the development process.

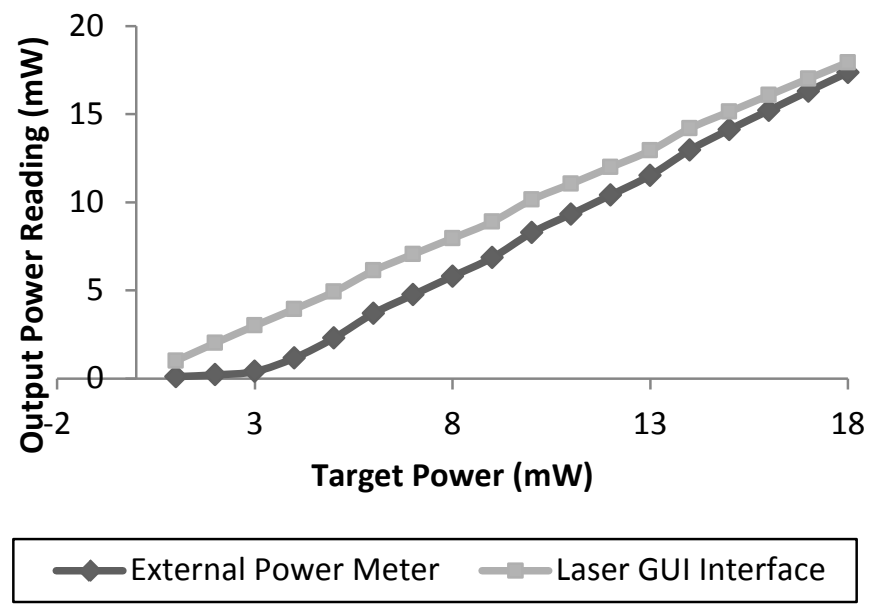

Figure 4.13. Laser output power plot that shows a discrepancy between actual output power and that seen on the laser GUI interface, especially at the lower power levels $(<5 \mathrm{~mW})$.

Through these experiments, the primary questions concerning the characterization of the LDW system have been addressed. Writing parameters for creating guides approximately $50 \mu \mathrm{m}$ in width is a standoff distance of $45 \mathrm{~mm}$ at the standard recommended $100 \%$ dose, or $1200 \mathrm{~mJ} / \mathrm{cm}^{2}$. Laser output power is not critical, though higher levels result in higher throughput and ensure a more complete cure.

\subsection{Waveguide Support Structures}

\subsubsection{Basic Structures}

\subsubsection{Turns, Spiral, Crossings}

Having characterized the basic parameters of the LDW process, a series of patterns was fabricated to determine the ability of the system to create various important waveguide shapes, and to identify the overall quality of those structures.

Basic waveguide structures of importance consisted of $90^{\circ}$ turns (1-10 mm bend radius), a one meter long spiral to measure attenuation loss over a long length, and a crossing pattern to determine optical loss in intersecting waveguides (Figure 4.14). Before these 
structures could be effectively evaluated, the direct write system needed to be tuned to achieve the desired pattern results.

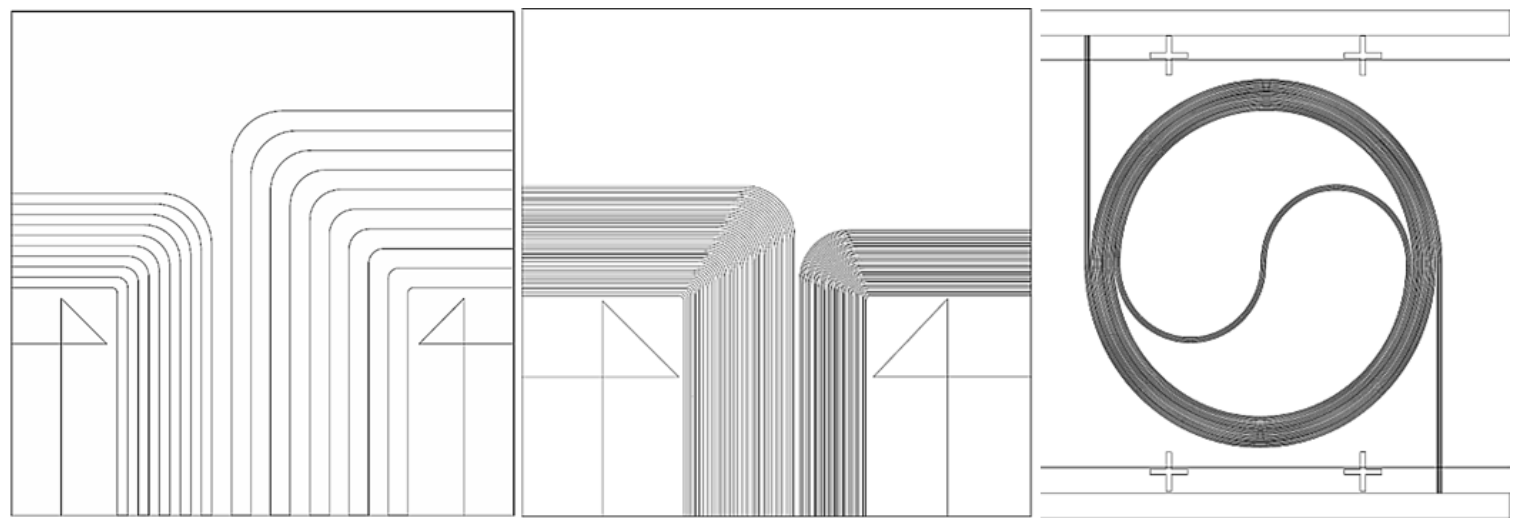

Figure 4.14. CAD drawings for waveguide exposure patterns. $90^{\circ}$ turns, $1-10 \mathrm{~mm}$ radius with $500 \mathrm{um}$ pitch (left), $250 \mathrm{um}$ pitch (center), and $1 \mathrm{~m}$ spiral with $30 \mathrm{~mm}$ maximum radius and $6 \mathrm{~mm}$ minimum radius(right).

\subsubsection{Shutter Integration}

When writing the waveguide crossing pattern, it was observed that the waveguide structures were blending together in certain areas of the pattern. By default, the motion platform decelerates to zero between all movements to maintain positional accuracy, as well as to reduce jerking, or overloading the linear magnetic axis motors. By slowing the write speed at the ends of motion, the uncured material becomes increasingly overdosed until the waveguide features are effectively blended together (Figure 4.15). This obstacle was overcome with the introduction of a mechanical shutter (Uniblitz Model 26L2AOX5 by Vincent Associates) and timer control box (Uniblitz 310 B by Vincent Associates), which were integrated with the drive controller for the motion platform (EDU171 Npaq by Aerotech) to modulate the laser ON/OFF sequences. Using this setup, motion would be allowed to continue beyond the point where a waveguide structure should terminate, with the shutter mechanism closing to blank exposure in this unwanted region (Figure 4.16). The axis would then be able to decelerate to zero without UV exposure. This solution was developed and implimented, but no further demonstration was conducted to illustrate the elimination of feature blending. The mechanical shutter was also useful for flash exposing the uncured waveguide material to create vertical waveguide structures. 


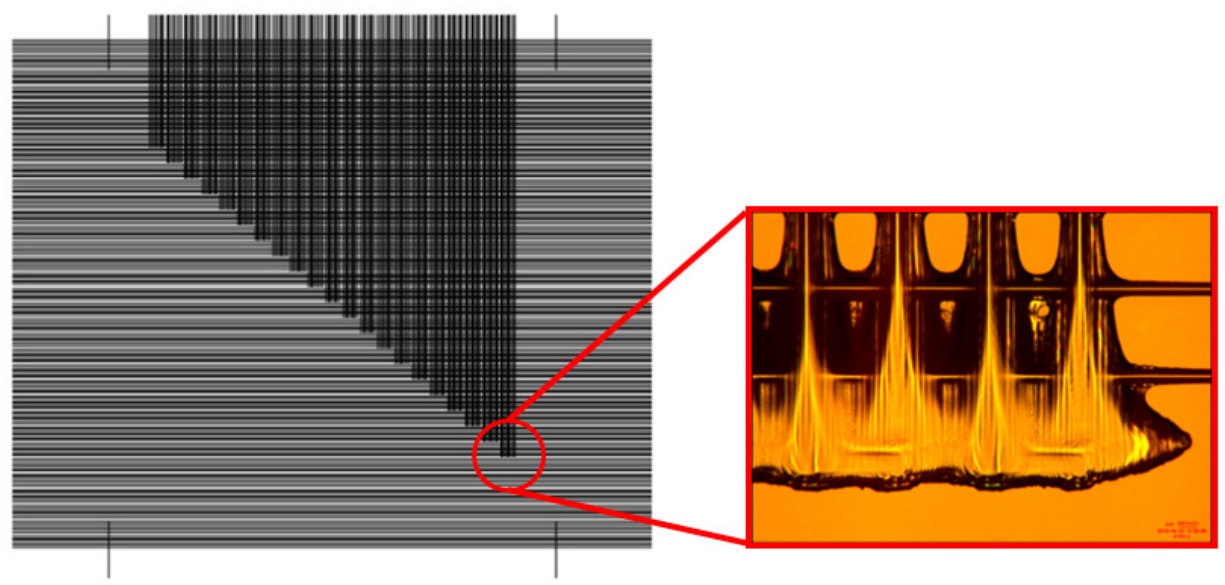

Figure 4.15. Crossing pattern shows that some standard CNC system features are not ideal for waveguide patterning. General operation requires system to decelerate to zero velocity between movements, over-curing the material.

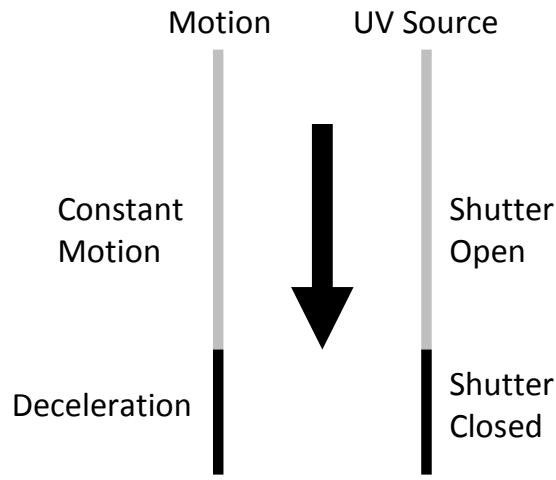

Figure 4.16. Shutter operates in conjunction with motion platform to prevent over-curing due to deceleration.

\subsubsection{Motion System Tuning}

While fabricating the waveguide bends, it was observed that "tight" tuning of the motion platform was critical for writing small radius turns. It has been demonstrated that dose plays a significant role in the patterned width of a waveguide, so it is critical that the system maintain a constant linear speed during the write process. With a $90^{\circ}$ turn of small radius, the motion platform must decelerate to zero from full speed in one axis of motion, while accelerating from zero to full velocity in the orthogonal axis, all in the length of the turn ( $\sim 1.57 \mathrm{~mm}$ for a $1 \mathrm{~mm}$ radius turn for example). This is obviously more difficult at higher writing speeds. The difference between a poorly tuned system and a properly tuned system can be seen in Figure 4.17, where the waveguides on the left were written with an under-damped system and the waveguides on the right with a critically damped system. The laser patterned waveguide turns passed visual inspection, but their performance was 5-10 dB worse than that of the lithographically patterned turns 
(as was the spiral), and at times performance was not measurable. This was due to the slightly elliptical shape of the beam resulting in waveguide widths that varied depending on the direction of writing. The problem was exacerbated by the aberrations present in the beam, which result in non-uniform curing of the material within the spot area.
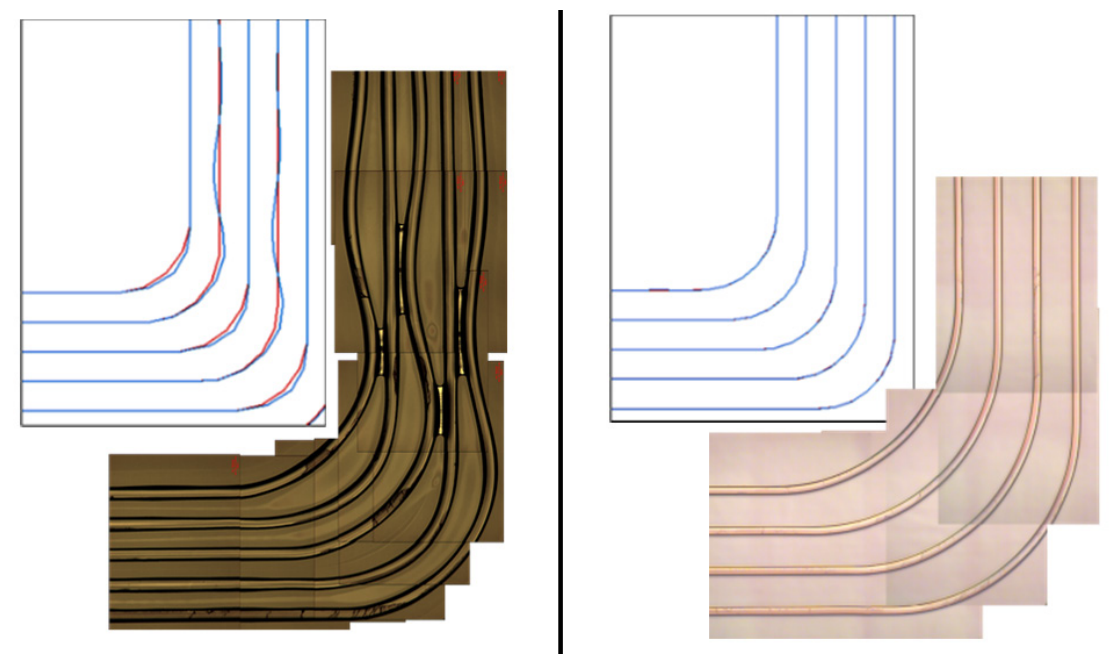

Figure 4.17. Proper system tuning is critical to waveguide performance. The system on the left is under-damped, resulting in oscillating waveguides, while the system on the right is properly tuned.

\subsection{Laser Source Quality}

The performance of the laser patterned waveguide turns were far below the measured results of the standard lithographically patterned waveguide turns (Figure Blah/performance chart), undermining the viability of the laser direct write tool as a method for fabricating waveguide networks. To improve upon these results, methods for improving the shape and quality of the laser source were investigated, which included spatial filtering of the output beam, as well as coupling the beam into a single mode fiber.

\subsubsection{Spatial Filtering}

Spatial filtering is a method of "cleaning up" a laser beam by removing the unwanted spatial noise, or the ring patterns observed from the beam scans (Figure 4.10). The process of spatial filtering (Figure 4.18) begins with focusing the laser output beam down to pass it through an aperture. The aperture allows only the central Gaussian spot to pass through, while the side fringes (noise) are blocked. After the beam passes through the aperture, it begins to expand, requiring a final lens set to collimate and refocus the beam to the desired spot size. The described setup was successfully implemented on an optical bench (Figure 4.19), and the output shape and power distribution of the laser source was significantly improved (Figure 4.18). 

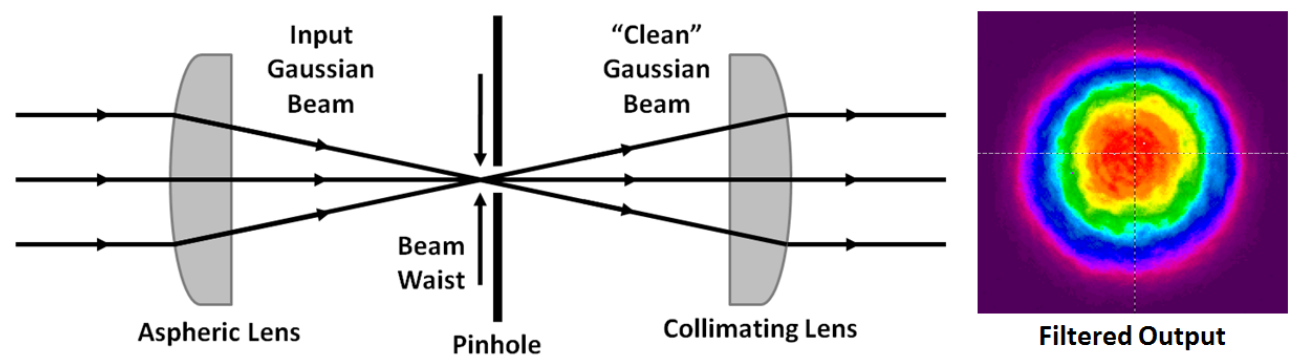

Figure 4.18. Spatial filter schematic and resulting output. Ring patterns have been removed and elliptical beam shape is now circular with more uniform power distribution.

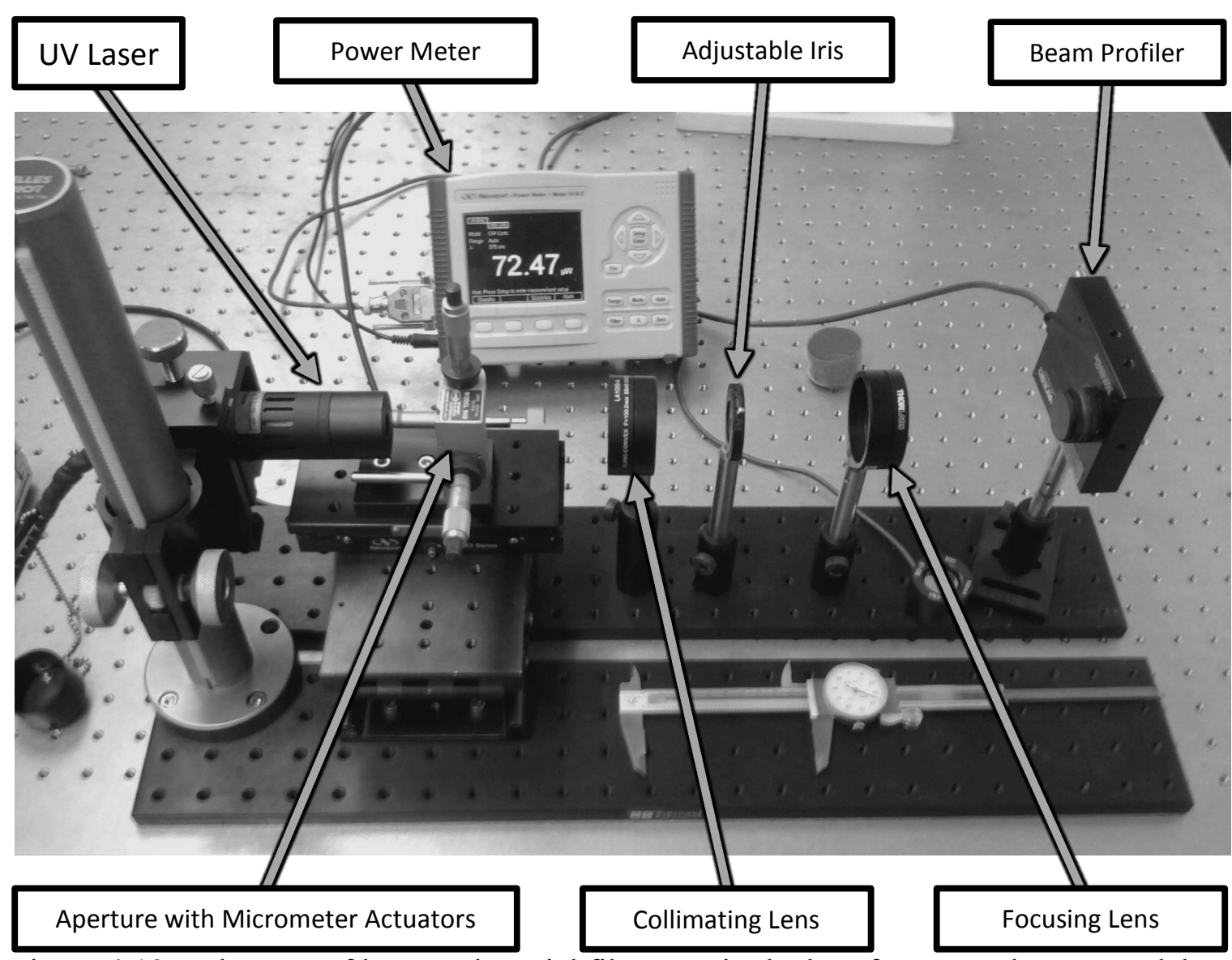

Figure 4.19. Lab setup of laser and spatial filter required a lot of space and was very labor intensive to achieve proper alignment between all of the components.

Spatial filtering was effective for improving the beam shape and quality, but had drawbacks. The additional required optics, along with aperture masking process, significantly decreased the final output power of the beam to $\sim 30-40 \%$ of original power, which would decrease throughput, as it would require slower write speeds. More adverse is the complicated nature of the setup, which would be difficult to integrate into the direct write system. The filter assembly is heavy and cumbersome, which would have 
made it difficult to mount onto the Z-axis of the direct write tool. Also, the filter configuration is labor intensive to set up, as it requires precise alignment between the initial lens, the aperture, final focusing optics, and an iris to block out any lower frequency noise at the outside of the beam. Establishing this alignment and maintaining and validating it with frequent use would be a very challenging task that would significantly decrease production, therefore it was not used.

\subsubsection{Single Mode Fiber Coupled Laser for Beam Clean-up}

A method better suited to the functionality of the direct write tool was to couple the laser source into a single mode fiber (SMF). A Toptica iBeam Smart 375-S UV laser (18 $\mathrm{mW}, 379 \mathrm{~nm}$ measured peak wavelength) was coupled into a UV grade single mode fiber with the Toptica SmartDock fiber coupler, making it easy to mount to the Z-axis. While a significant amount of power is lost ( $40 \%$ of original power lost) by coupling into such a small diameter fiber core (mode field diameter of 2-3 $\mu \mathrm{m}$ ), the output is a circular beam shape with a clean Gaussian power distribution. The radial symmetry of the beam allows uniform writing in all directions, and the uniform power distribution means the resulting structures will not have irregular shapes and features as a result of beam aberrations. Having the beam output from a fiber saved space and presented desirable mounting flexibility on the Z-axis (Figure 4.20).

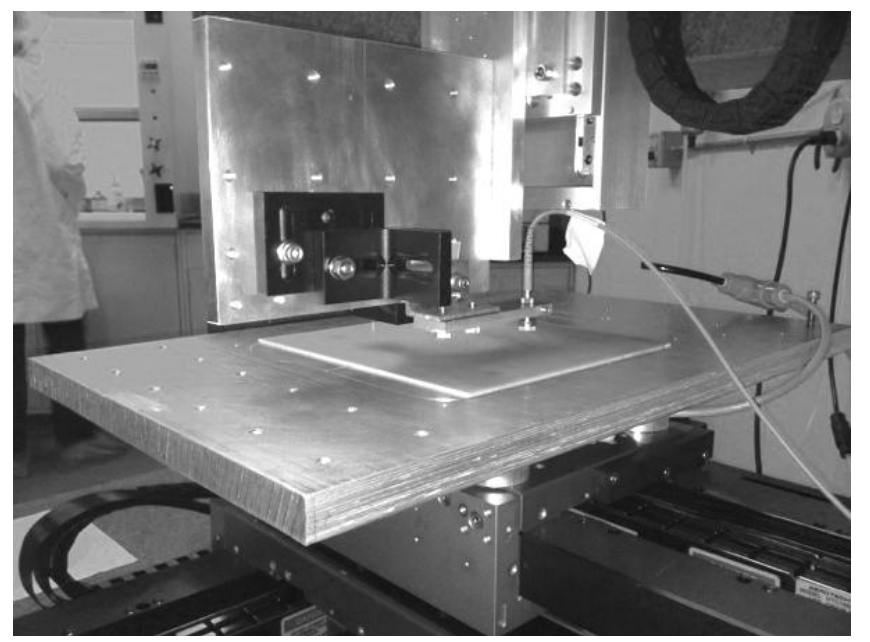

Figure 4.20. A Toptica iBeam Smart 375-S UV laser $(18 \mathrm{~mW}, 379 \mathrm{~nm}$ measured peak wavelength) was coupled into a UV grade single mode fiber with the Toptica SmartDock fiber coupler, making it easy to mount to the Z-axis. 
This method of beam shaping does not come without challenges. The output beam is divergent upon exiting the single mode fiber, which is less than $10 \mathrm{um}$ in diameter. This results in very short standoff distances for patterning $50 \mu \mathrm{m}$ wide waveguides (Figure 4.21). The single-mode fiber has a mode-field diameter (MFD) of 2-3 $\mu \mathrm{m}$, which was used to calculate the required standoff distance to achieve a spot that is $50 \mu \mathrm{m}$ in diameter.

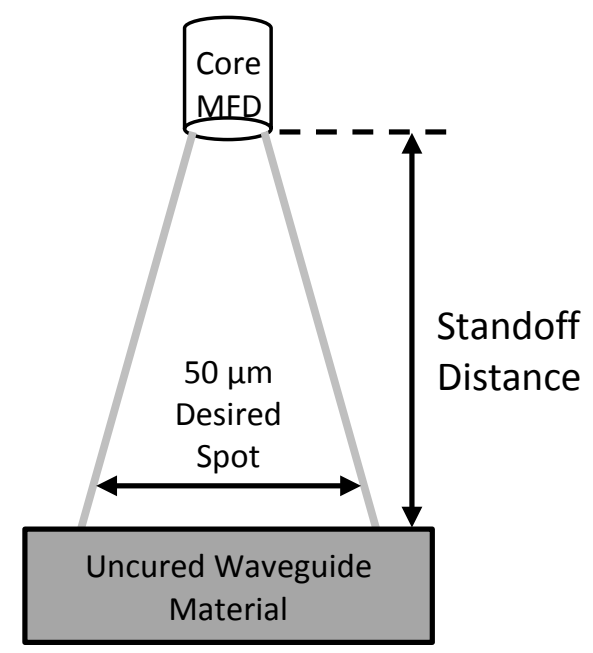

Figure 4.21. Beam divergence necessitates short standoff distance.

The initial beam diameter exiting the fiber, $2 \mathrm{w}_{0}$, can be set equal to the mode-field diameter of $2 \mu \mathrm{m}$ (resulting in the worst case condition of shortest standoff distance), and a standoff distance $(\mathrm{z})$ which results in a beam radius of $25 \mu \mathrm{m}$ can be found according to Equation 4.4 and Equation 4.5 (68).

$$
w(z)=w_{0} \sqrt{1+\left(\frac{z}{z_{R}\left(w_{0}\right)}\right)^{2}}
$$

where

$$
z_{R}\left(w_{0}\right)=\frac{\pi}{\lambda} w_{0}^{2}
$$

Using these values, the standoff distance required for a $50 \mu \mathrm{m}$ diameter spot was determined to be $207 \mu \mathrm{m}$. This is a very short standoff distance with little tolerance, as $100 \mu \mathrm{m}$ vertical deviation results in a $25 \mu \mathrm{m}$ diameter change. The poor results of waveguide turns written with a slightly elliptical beam illustrate that small variances in waveguide width can degrade optical performance. Slight changes in substrate or layer topology present a significant problem with such tight vertical tolerances. To evaluate the writing ability of single mode fiber output and to validate the standoff distance calculations for a single mode fiber, a set of $90^{\circ}$ waveguide turns $(1-10 \mathrm{~mm}$ bend radius, Figure 4.14) were patterned at standoff distances of $400 \mu \mathrm{m}, 300 \mu \mathrm{m}$, and $200 \mu \mathrm{m}$ with a 
dose of $1200 \mathrm{~mJ} / \mathrm{cm}^{2}$. The resulting waveguides were $95 \mu \mathrm{m}, 70 \mu \mathrm{m}$, and $48 \mu \mathrm{m}$ wide, respectively, which correlates very well with the expected values of 97,72 , and $48 \mu \mathrm{m}$. The $48 \mu \mathrm{m}$ waveguides were tested and demonstrated great improvement over the previous laser written waveguides (Figure 4.22). The waveguide dimensions were consistent on either end of the turn, meaning that the laser was writing uniformly with each axis of the beam.
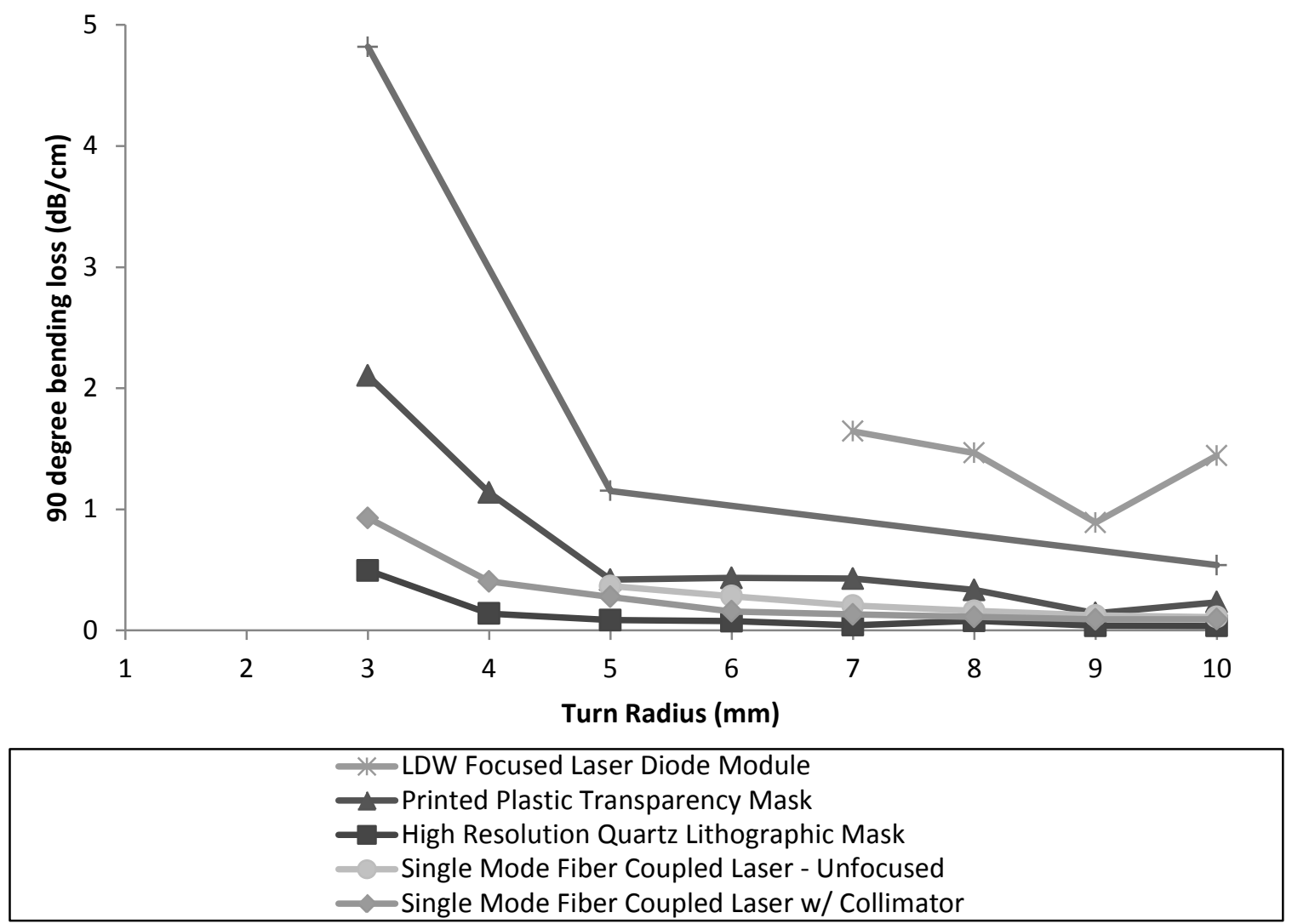

Figure 4.22. SMF coupled laser with collimator provides best direct write results, second overall to lithographically produced waveguides.

The fiber face made contact with the waveguide material at the perimeter of the substrate where there was edge beading, proving that the short standoff distance was a significant obstacle. This was overcome by utilizing a commercially available, adjustable aspheric fiber optic collimator (Thorlabs CFC-2X-A). The compact collimator was easily integrated using a standard FC connection, with the output beam shape being modified by adjusting the distance between the fiber end-face and the included aspheric lens. The fiber optic collimator provided a longer beam waist resulting in more stable writing conditions and was accomplished without distorting the beam (Figure 4.23) 

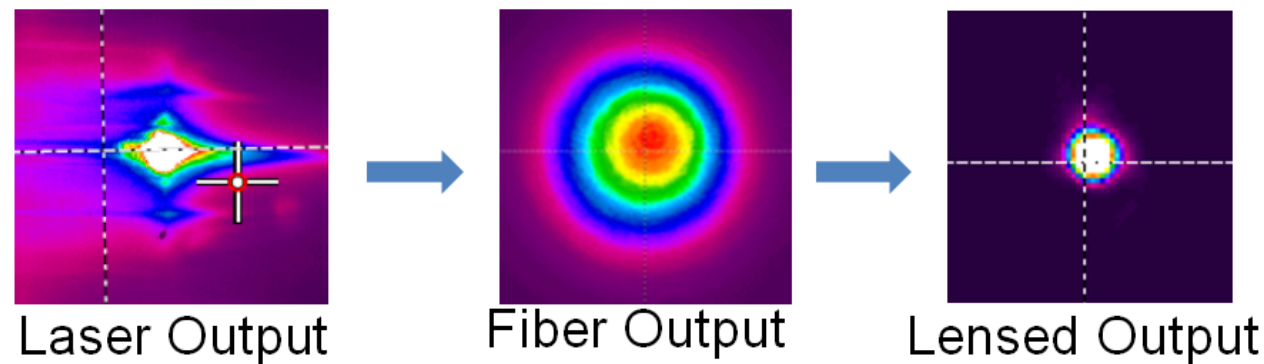

Figure 4.23. Beam outputs for laser diode, single mode fiber coupled source, and lensed output.

The standoff distance and write speed characterization work was repeated with this focused beam to determine the proper writing parameters for this new beam profile, and a set of $90^{\circ}$ waveguide turns were fabricated to measure performance. The performance of the waveguides produced with the collimated single mode fiber setup improved over those written with no collimation, and were significantly better than those produced with the original lens focused diode source. The turns patterned with the collimator performed the best of all laser written turns, and approached the quality of the standard lithographically produced guides (Figure 4.22). Straight waveguides patterned with this setup exhibited losses of $0.047 \mathrm{~dB} / \mathrm{cm}$, which is nearly equal to the $0.043 \mathrm{~dB} / \mathrm{cm}$ seen in the lithographically patterned waveguides.

Another benefit of using the adjustable collimator was the ability to direct write waveguide structures at single mode sizes. The collimator was adjusted so that the distance between the aspheric lens and the fiber face was at a minimum and the write speed and standoff distance characterization experiment was repeated on a thin layer $(<10$ $\mu \mathrm{m})$ of uncured core material. By lowering the dose delivered to the material (113 $\mathrm{mJ} / \mathrm{cm}^{2}$, calculated using a $1 \mathrm{~mW}$ output power and $20 \mu \mathrm{m}$ assumed beam diameter), waveguides were patterned which were $\sim 8 \mu \mathrm{m}$ wide (Figure 4.24 ), proving that the SMF coupled laser writing setup can rapidly and dynamically pattern small enough features for single mode operation. 


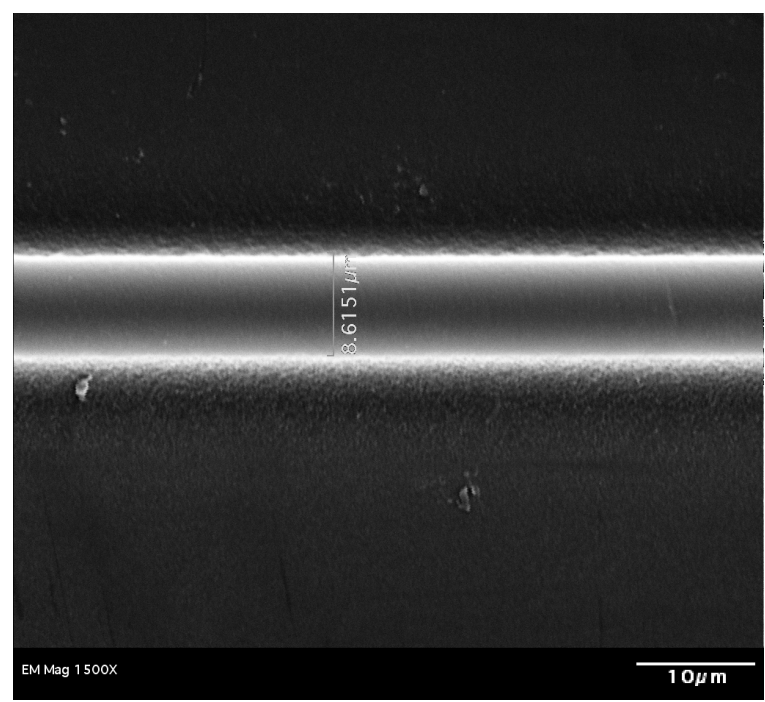

Figure 4.24. Waveguides patterned at a standoff distance of $1 \mathrm{~mm}$ and a feed rate of 57 $\mathrm{mm} / \mathrm{sec}(113 \mathrm{~mJ} / \mathrm{cm} 2$ energy dose $)$. Waveguide width is $8.62 \mu \mathrm{m}$.

\subsubsection{Optical Via Structures}

\subsubsection{Angled Waveguides for Reflector}

In conjunction with the basic waveguide structures, a pair of more complex shapes was patterned with the intent of forming a laser patterned optical via. This would provide a method of turning the optical signal "out-of-plane" for vertical coupling.

The first patterned feature was a waveguide written on an angle to give it a rhombusshaped cross section (Figure 4.25). The angled sidewalls could serve as reflector faces for a laser patterned via structure. These features were patterned by changing the incident angle of the UV laser source from the typical $90^{\circ}$ (with respect to substrate surface) to $45^{\circ}$.
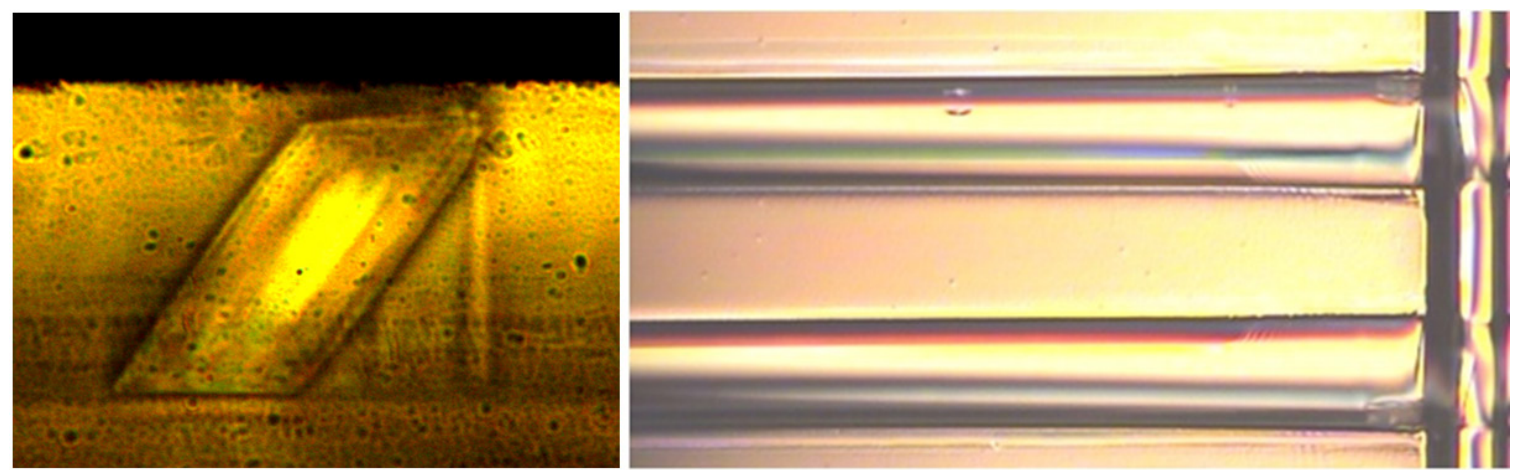

Figure 4.25. Angled waveguide structure (left) serves as reflector face for orthogonal waveguides (right). 


\subsubsection{Vertical Waveguide Structures}

The second feature of this set was a high aspect ratio vertical cylindrical structure. The purpose of these structures would be to contain the optical signal in the vertical direction, acting as a short "light pipe". The CNC operation of the direct write tool made it possible to precisely align these structures with previously written waveguides, seen in Figure 4.26. An important observation was how the ring patterns observed in the initial beam scans directly transferred to the waveguide material (Figure 4.27). While similarity between the beam and the resulting structure is impressive, the irregular oval shape and heterogeneous power distribution of the beam are undesirable for uniform patterning in all directions of motion. Waveguides patterned in one axis of motion would result in a different size as those patterned in the other axis of motion, illustrated by the poor performance of the waveguide turns.
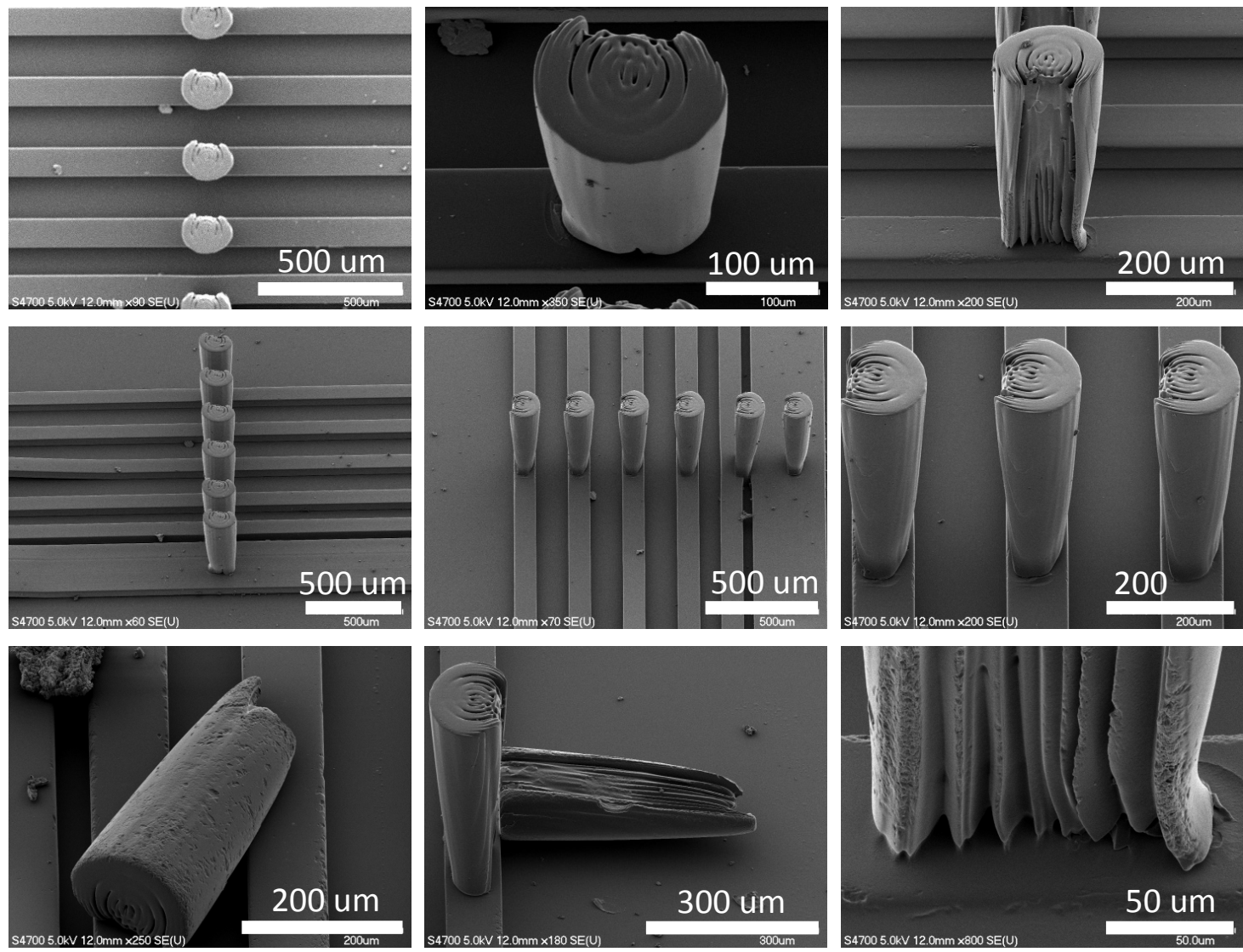

Figure 4.26. SEM images of spot cured waveguide material to form high aspect ratio structures. 


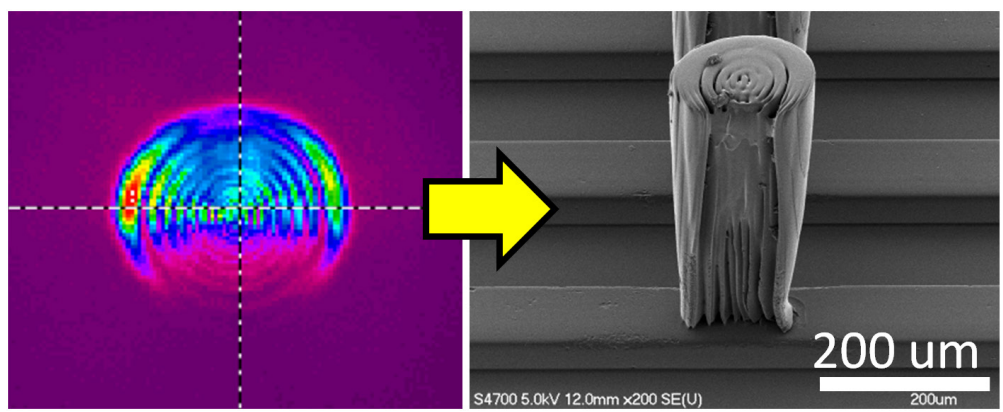

Figure 4.27. Aberrations in beam negatively affect patterning process.

\subsection{Laser Direct Write Photo-patterning Conclusions}

A single mode fiber coupled laser provided excellent beam symmetry and uniform power distribution, resulting in direct write patterned waveguides that were comparable in quality to those produced using standard lithographic practices $(0.047 \mathrm{~dB} / \mathrm{cm}$ for laser written waveguides compared to $0.043 \mathrm{~dB} / \mathrm{cm}$ seen in the lithographically patterned waveguides). Straight waveguides, as well as waveguide turns were successfully patterned at multimode and single mode sizes, and the process for writing these waveguides was well characterized and repeatable. Support structures such as angled reflectors and vertical posts were produced, showing the versatility of the laser direct write tool. 


\section{Chapter 5. Direct Dispense Material}

\section{Deposition}

The second main function of the direct write tool is the direct dispensing of the uncured waveguide material in broad films or distinct beads on the substrate surface. With the high cost of waveguide material, this process is an improvement over the traditional method of spinning, where most of the deposited material is spun off as waste or subsequently washed away after only a small portion is cured. This process can also handle much larger substrates than the 4-6 inch samples that a typical spinner can accommodate. This makes it more suitable when dealing with circuit board size substrate panels that can be up to $457 \mathrm{~mm} \times 610 \mathrm{~mm}$ (18 inches x 24 inches). The direct dispensing of material is achieved with a pneumatically-driven, syringe dispense device (Ultimus V by Nordson-EFD) which uses commercially available Luer-lok style syringe needles to dispense materials over a range of viscosities. This tool can be used in conjunction with the laser direct write system, where either a broad layer or a narrow band of waveguide material is deposited and device feature definition is provided by the UV laser. Another approach for the direct dispense tool is to directly deposit single waveguides which meet their target dimensions and thus do not require precise UV exposure or development to provide definition. Using the direct dispense approach, the roughness of the resulting waveguides would not be influenced by mask quality or beam shape, but rather is based on the surface tension of the uncured material.

Whether depositing blanket layers or distinct beads, either approach can result in dramatic material savings through the reduction of waste when compared to the standard approach of spinning raw siloxane:

- 40 - 60 times less material used per layer (as compared to manufacturer's recommended amount required for spinning). Discrete waveguide deposition offers even greater savings, as it leaves zero waste and eliminates a development step.

- This material savings translates to a sample cost of tens of dollars instead of hundreds (for a 3 layer sample consisting of clad-core-clad on a $100 \mathrm{~mm}$ square substrate at a material price of many thousands of dollars per liter).

\subsection{Process Summary}

The writing process was followed for both the deposition of blanket layers and the deposition of distinct waveguides. The first step, after the uncured material has been loaded into the syringe barrel for dispensing, was to obtain the vertical reference between the dispense tip and the substrate surface, accomplished through physical contact. The 
syringe assembly was loosely affixed in its mount by inserting the syringe tip and reservoir barrel with the clamp screw left untightened. This left the assembly with freedom of movement in the vertical direction (Figure 3.6). The vertical axis was slowly lowered until the syringe tip made contact with the substrate surface. The clamp screw was then tightened and the current vertical position of the axis was recorded from the $\mathrm{CNC}$ interface to serve as a reference point. By raising the vertical axis by known amounts, the standoff distance of the syringe tip with respect to the substrate surface is accurately identified. The syringe was oriented $90^{\circ}$ to the substrate surface to provide uniform dispense conditions independent of writing direction.

After the dispense height was established, the write speed and dispense pressure were set to the desired values (all pressure settings are in PSI since the equipment used these units). The dispense pressure remained applied as the syringe traveled back and forth over the length of the substrate, jogging orthogonally at the desired line pitch. Once dispensing was complete, the substrate was subjected to the standard pre-bake, UV exposure, then post bake to cure the dispensed material, followed by rinsing away excess if necessary.

\subsection{Writing Parameters}

Fluid mechanic principles were used to identify the writing parameters for direct dispensing and to understand how they related to the written structures. Because the waveguide material is a non-Newtonian fluid, Bernoulli's Equation for steady flow (Equation 5.1, Figure 5.1) was used only as a guide for understanding parameter relationships. By this approach, the parameters which affect material flow were material density, dispense pressure, and cross sectional area of the flow channel (needle tip selection). Understanding the relationships between these variables was important when determining the system requirements for the direct dispense tool.

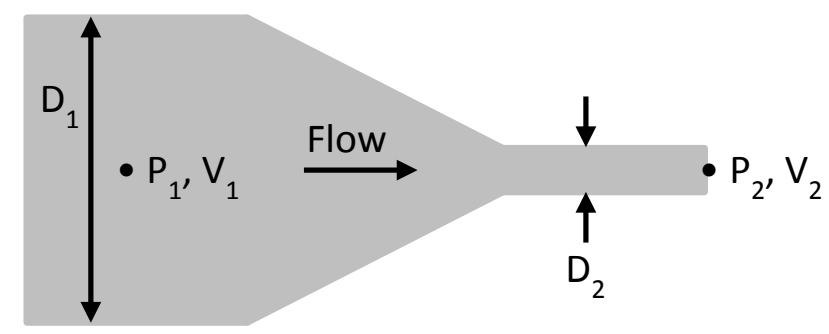

Figure 5.1. Steady flow diagram of dispense process. Material flows from a large reservoir out through a small diameter needle, increasing in flow velocity along the way.

$$
P_{1}+\frac{1}{2} \rho V_{1}^{2}+\rho g h_{1}=P_{2}+\frac{1}{2} \rho V_{2}^{2}+\rho g h_{2}
$$

Where $\mathrm{P}$ is pressure, $\rho$ is material density, $\mathrm{V}$ is the instantaneous flow velocity, $\mathrm{g}$ is gravity, and $\mathrm{h}$ is the height. Because the contribution of the gravitational component is 
negligible, and because the flow velocity at the needle exit is so much greater than the velocity within the syringe barrel (Equation 5.2), the equation can be simplified (Equation 5.3).

$$
\begin{gathered}
V_{2} \gg V_{1} \\
\Delta P=\frac{1}{2} \rho\left(V_{2}^{2}\right)
\end{gathered}
$$

For steady conditions where the flow rate (Q) is constant, the cross sectional area (A) and flow velocity at any two locations are related by:

$$
Q=A_{1} V_{1}=A_{2} V_{2}
$$

Where the area is a function of needle diameter (D) according to:

$$
A=\frac{1}{4} \pi D^{2}
$$

This shows that using a smaller needle diameter or a material with a higher density results in a decreased flow rate for a fixed pressure, and vice versa. The dispense process is also impacted by material viscosity, or the resistance of the material to flow. Poiseuille's equation (Equation 5.6) shows that the volumetric flow rate is inversely proportional to viscosity:

$$
Q=\frac{\Delta P \pi d^{4}}{128 \mu L}
$$

This relationship further illustrates how proper selection of material blend (density and viscosity) and dispense tip size is important.

\subsubsection{Material Blend}

Material blend is an important consideration because it directly affects the density and viscosity of the material, and changes the requirements of the other parameters relating to flow rate: needle size and dispense pressure. The uncured waveguide material is diluted in toluene and is supplied from the manufacturer with a "percent non-volatile content" rating (\%NVC). Material density and viscosity are based on the $\% \mathrm{NVC}$ of the material, with viscosity values for specific blends provided by the manufacturer (Figure 5.2). If the material viscosity is too low, it can flow unrestrained from the needle resulting in a loss of process control. Conversely, if the viscosity is too high, the pressure required to extrude the material from the small dispense tips can exceed the available supply pressure (80 PSI) or the safety rated pressure of the dispense components (100 PSI). The measured density of the standard blend of material $(70 \% \mathrm{NVC})$ was 1.1 grams $/ \mathrm{mL}$ for the core and 1.0 grams $/ \mathrm{mL}$ for the clad. The corresponding viscosity values were provided as $91.2 \mathrm{cP}$ for the core and $14.3 \mathrm{cP}$ for the clad. 


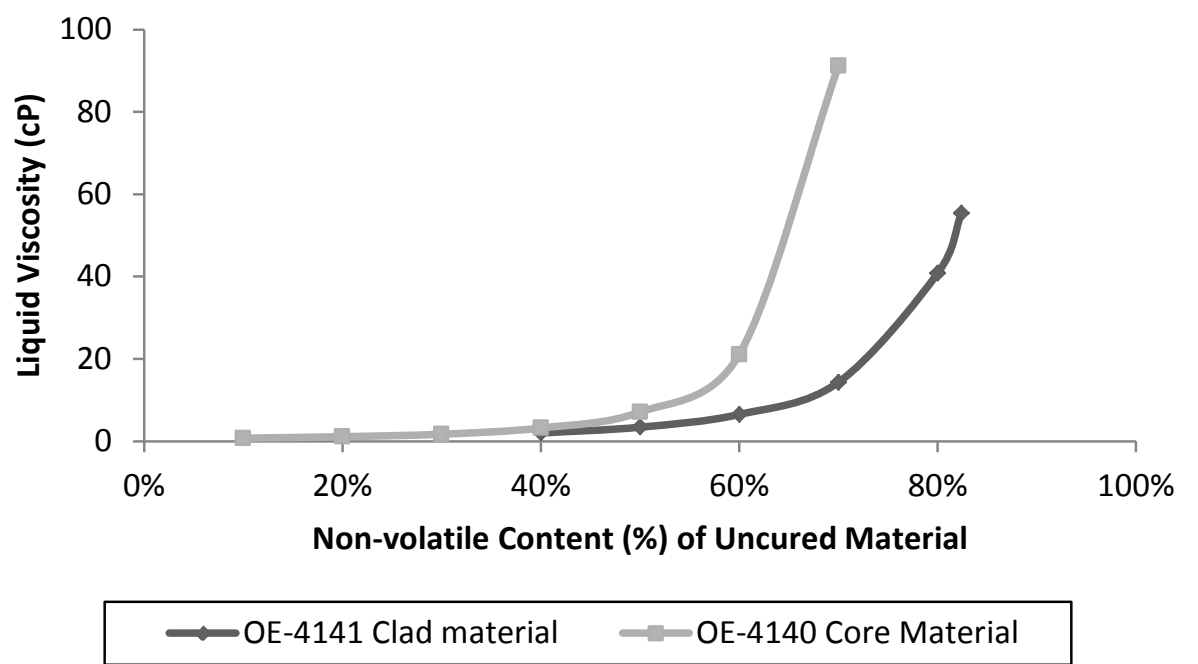

Figure 5.2. Core material with non-volatile contents of $70,75,80,85$, and $90 \%$ currently available from manufacturer.

\subsubsection{Dispense Tip Size}

The size of the dispense tip influences the required dispense pressure, and has a direct impact on the size of the deposited material bead, so proper selection for the intended application is important. For the deposition of discrete waveguides, small tips that are close to the intended waveguide dimensions are required. For blanket layers, broad coverage is important, so a larger needle is desirable as it allows for higher throughput at lower dispense pressures. A range of disposable tip sizes are commercially available, but primary needles of use were the larger 21 gauge needle $(510 \mu \mathrm{m}$ ID) for the deposition of thick blanket layers $(\geq 50 \mu \mathrm{m})$, the 32 gauge needle $(110 \mu \mathrm{m}$ ID) for thinner blanket layers $(25-75 \mu \mathrm{m})$, and the 33 gauge needle $(110 \mu \mathrm{m} \mathrm{ID})$ for the deposition of discrete waveguides (Table 5.1). The 33 gauge needle was chosen for its tapered outside profile, resulting in a smaller outer diameter (Figure 5.3).

Table 5.1

Dimensions for tips used in direct dispense process.

\begin{tabular}{|c|c|c|c|c|}
\hline Gauge & \multicolumn{2}{|c|}{ Inner Diameter } & \multicolumn{2}{c|}{ Outer Diameter } \\
\hline$(\#)$ & $(\mathrm{mm})$ & $($ in $)$ & $(\mathrm{mm})$ & (in) \\
\hline 21 & 0.510 & 0.020 & 0.820 & 0.032 \\
32 & 0.110 & 0.004 & 0.240 & 0.009 \\
33 & 0.110 & 0.004 & 0.210 & 0.008 \\
\hline
\end{tabular}



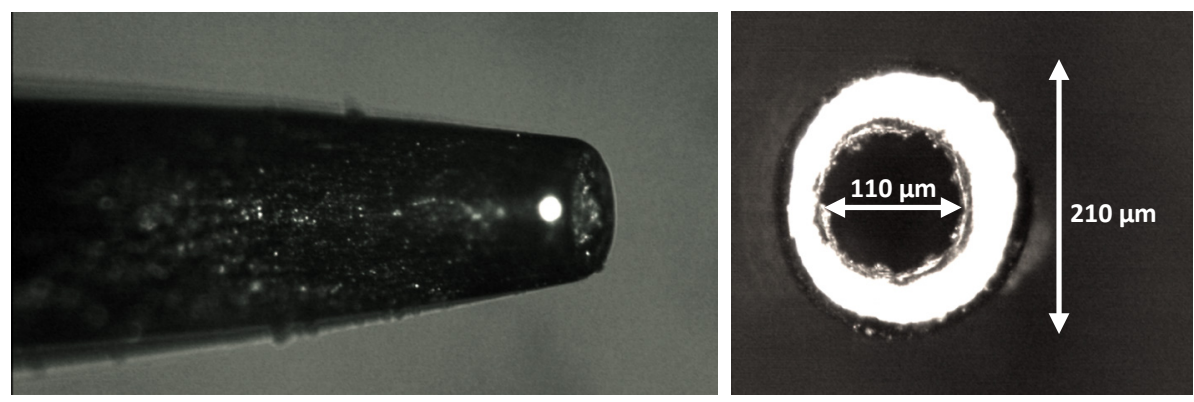

Figure 5.3. 33 gauge $(210 \mu \mathrm{m}$ outer diameter, $110 \mu \mathrm{m}$ inner diameter $)$ stainless steel needle tip, side view (left, 50x) and end view (right, 100x).

Needles with smaller inner diameters are also available, down to $0.5 \mu \mathrm{m}$ in the form of pre-pulled glass pipette tips (Figure 5.4), and $50 \mu \mathrm{m}$ in stainless steel. These smaller dispense tips would create smaller structures, but would require much higher pressures and tighter tolerances in the writing process to successfully produce continuous waveguides with the current waveguide material.
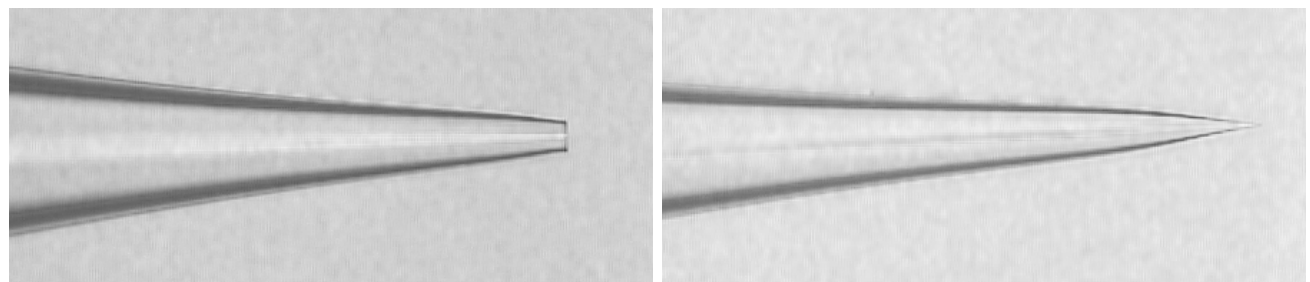

Figure 5.4. Pre-pulled glass needle dispense tips: $30 \mu \mathrm{m}$ inner diameter (left) and $2 \mu \mathrm{m}$ inner diameter (right).

\subsubsection{Dispense Pressure}

The dispense pressure directly influences the rate at which material is dispensed from the syringe system. The pressure must be just sufficient to ensure that there are no discontinuities in the dispensed bead, while still keeping feature sizes to a minimum. Dispense pressure was limited to 80 PSI by available supply pressure.

To simulate the process of dispensing siloxane waveguide material, an aqueous glycerin solution of $85 \%$ glycerin to $15 \% \mathrm{H}_{2} \mathrm{O}$ was prepared as a cost effective alternative that did not require the use of harsh solvents for cleanup. This blend was selected as it had a viscosity of $112.9 \mathrm{cP}$ which was comparable to the standard blend of waveguide core material (70\%NVC, $91.2 \mathrm{cP})$ (Hodgman, 1948). The solution was syringe dispensed from a 21 gauge needle into a beaker for a duration of 30 seconds. The dispensed mass was then weighed and the resulting value was divided by the elapsed time to obtain a mass flow rate for the material. The mass flow rate was then converted to volumetric flow rate by dividing by the material density. This process was repeated over a range of dispense pressures to determine volumetric flow rate as a function of applied pneumatic deposition pressure. This exercise illustrated that the flow rates for the aqueous glycerin 
solution were linear in relation to the deposition pressure, seen in Figure 5.5. This was desirable from a characterization standpoint as it points to good repeatability and predictability.

After material dispensing was tested with glycerin, the next step was to proceed with siloxane waveguide core and cladding material. The same procedure for obtaining volumetric flow rates was conducted using clad and core material (70\%NVC for both). The clad material was characterized using a 21 gauge needle (Figure 5.5), as the cladding layers can typically be thicker than the core layers, and the core material was characterized for both a 21 gauge and a 32 gauge needle (Figure 5.5 and Figure 5.6). The results show that, as with the glycerin solution, the measured flow rates for both the cladding and core materials were linear in relation to the applied deposition pressure.

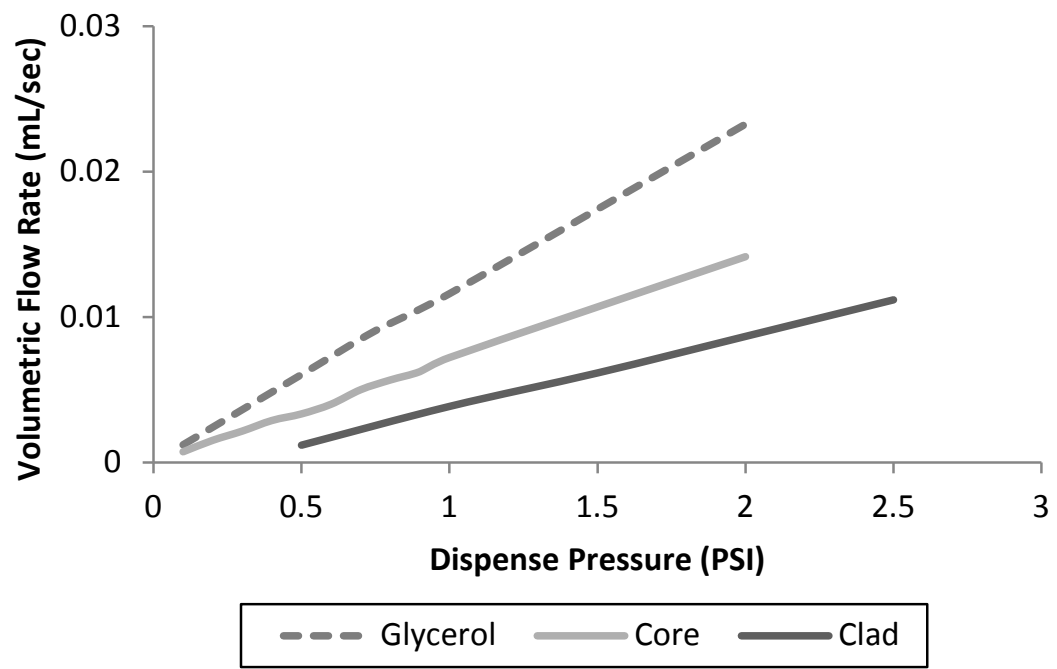

Figure 5.5. Flow rates of glycerol, core, and clad material through a 21 gauge needle as a function of applied pressure. 


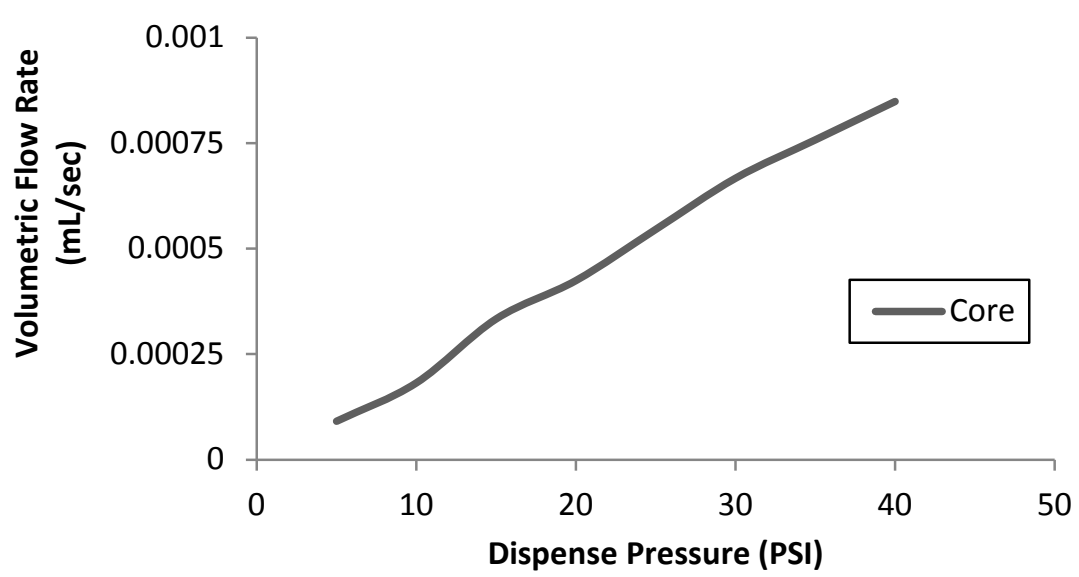

Figure 5.6. Flow rate of core material through a 32 gauge needle as a function of applied pressure.

\subsubsection{Write Speed}

In addition to the flow parameters, there are variables associated with the dynamic application of the dispensed material onto a substrate. One of these writing variables is write speed, which directly influences waveguide size. As with laser direct writing, faster write speeds result in smaller structures and vice versa. To obtain a starting point for writing $50 \mu \mathrm{m}$ waveguide structures, the flow rates for the waveguide material were used, according to Equation 5.7, to calculate write speed. During the dispense process, a liquid bridge forms between the syringe tip and the substrate surface due to the tackiness and surface tension of the material (Kai, 2008). This results in the material being drawn out of the syringe, with faster write speeds resulting in increased necking of this liquid bridge and ultimately smaller waveguide structures. If the write speed is too high, the liquid bridge will break, resulting in a discontinuous waveguide structure. Slower write speeds allow more material to accumulate over a fixed distance, resulting in larger waveguide structures.

$$
Q=A_{2} V_{2}=A_{\text {waveguide }} * \text { Write Speed }
$$

\subsubsection{Dispense Height}

The other variable associated with dynamically dispensing material is dispense height. While the other dispense variables contributed to overall waveguide size, dispense height proved to be the critical variable affecting continuity in the waveguide structure. If the dispense height is too high or too low, the size and shape of the resulting beads can be greatly impacted. Improper dispense height can also lead to discontinuities in the waveguide structure, significantly degrading optical performance. The effects of dispense height were isolated by holding each of the other dispense variables constant and varying the height of the needle from the substrate surface, starting on the order of the inner diameter of the dispense needle. The direct dispense tool was used to dispense high viscosity core material (90\% solids content) through a 33 gauge dispense tip, onto a 
cladded FR4 substrate. To successfully dispense this high viscosity material, the maximum available pressure of 80 PSI was applied, and the write speed was kept at a low rate of $10 \mathrm{~mm} / \mathrm{second}$. The dispense height was varied and the effects were observed, starting at $75 \mu \mathrm{m}$ separation and working closer.

Discontinuous waveguides and waveguides with periodic bulging were observed with a $75 \mu \mathrm{m}$ dispense height (Figure 5.7). The distance between the dispense tip and the substrate surface was too great for the material to make constant contact with the surface, causing the siloxane to build around the needle tip. This was a cyclical process, detailed in Figure 5.8.

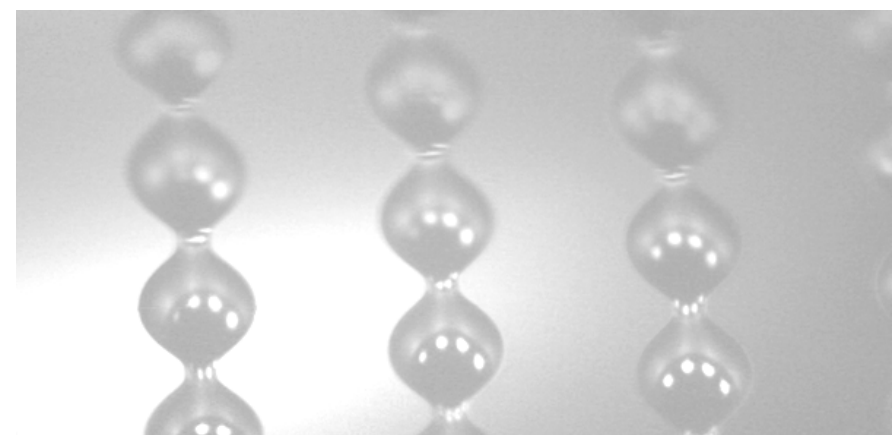

Figure 5.7. Dispense height of 75 um was too high from substrate, resulting in periodic bead patterns.

The uncured waveguide material was very tacky, and had a high surface tension. Figure 5.8(A) shows how these cohesive forces inhibit a droplet from overcoming gravity, causing it to grow and build around the needle tip. In Figure 5.8(B), the droplet grows until, in Figure 5.8(C), it is finally large enough to contact the substrate surface. When the droplet makes contact, the material adheres to the cladding and elongates as the needle moves across the sample surface. As the needle keeps moving, the material begins to neck until the material string is drawn out, and sometimes broken, after which the process begins again, seen in Figure 5.8(D).

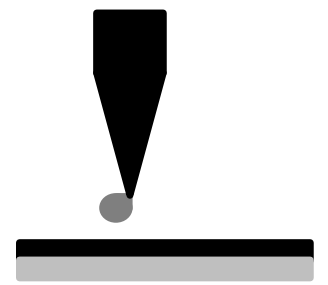

(A)

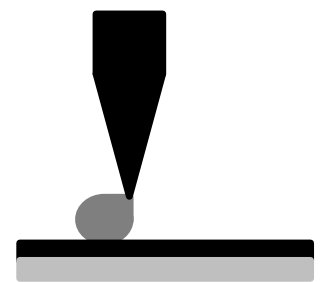

(B)

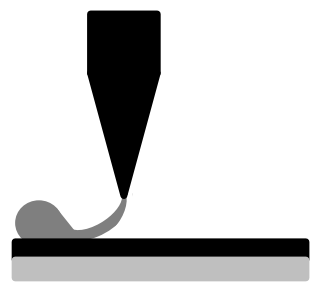

(C)

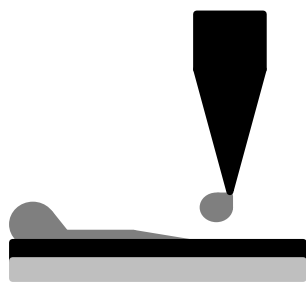

(D)

Writing Direction

Figure 5.8. Repetitive cycle observed in the dispensing process when the dispense needle is positioned too high off the substrate surface: (A) droplet forms on tip of needle (B) droplet grows in size until it contacts the substrate surface (C) droplet adheres to substrate 
and draws material out of needle (D) material necks until the material string is drawn out and sometimes broken, after which the process begins again.

Conversely, a $50 \mu \mathrm{m}$ dispense height was determined to be too small of a separation between dispense tip and substrate. Writing at these conditions resulted in wide and shallow guides that often appeared smeared or streaked (Figure 5.9). When the tip was too close to the surface, the material was not able to be drawn from the tip, but rather expanded out around the needle tip (Figure 5.10). As this accumulated material was swept across the substrate surface, a wide and shallow streaked structure was deposited.

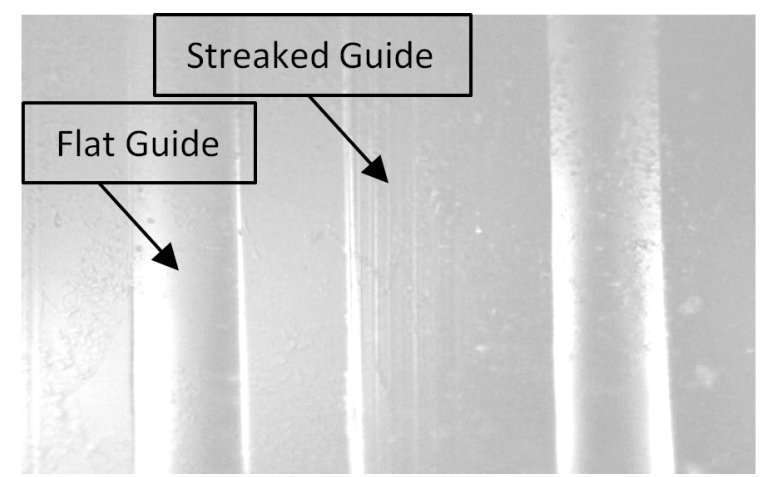

Figure 5.9. Dispense height of 50 um was too close to the substrate, resulting in flat and streaked waveguides.

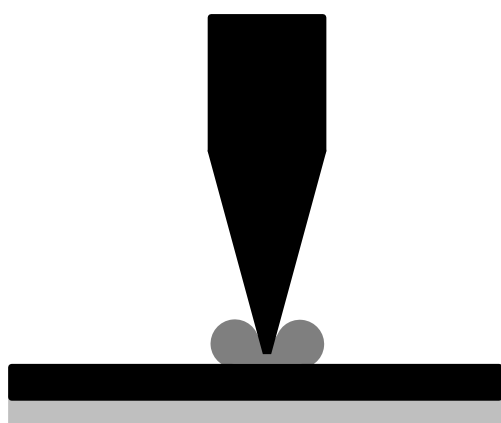

Figure 5.10. Dispense needle is too close to substrate surface, inhibiting free flow from the tip

Continuous guides were produced with no bulging or breaking using a dispensing height of $65 \mu \mathrm{m}$ (Figure 5.11). This shows that small differences in dispense height can significantly impact the resulting waveguides, revealing the sensitivity of this process. It is important to note that the specific dispense height values obtained in this exercise are valid only for the specific pairing of $90 \%$ NVC material blend and 33 gauge dispense tip. 


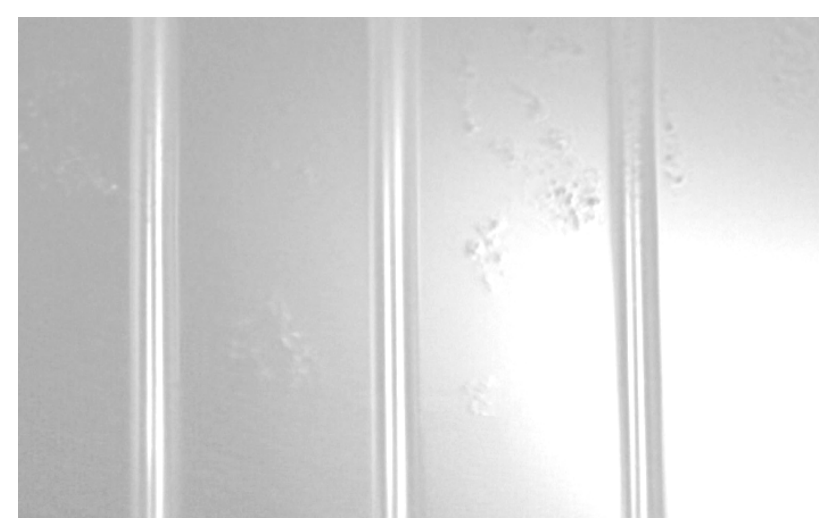

Figure 5.11. Dispense height of 65 um resulted in continuous and uniform waveguide structures that were $50 \mu \mathrm{m}$ in width

While the vertical axis of the direct write tool can be accurately controlled and can be held constant, any variance in the thickness of the sample fixture workplate, the FR4 substrate, or the lower waveguide cladding layer can drastically alter the resulting waveguide structures during the direct write process, as they all directly impact the dispense height. These experiments showed that micrometer variances can be detrimental to waveguide continuity, and that a solution is required if waveguides are to be reliably and repeatably fabricated in this manner.

\subsection{Dispensing Blanket Layers}

The process for dispensing blanket layers of clad and core material was to syringe deposit beads of material in straight lines at a controlled line pitch. The substrate was then baked to allow for the closely grouped beads of material to flow together, and then the material was flood exposed and final baked. The line pitch plays a role in the resulting layer thickness, as a closer pitch results in more material deposited per unit of area. Wider line spacing results in less total material being deposited and thinner layers. If the spacing is too wide however, the beads are unable to flow together, resulting in distinct beads rather than a uniform layer.

To characterize the layer deposition process, 70\% NVC core material was dispensed directly onto bare FR4 substrates with a 32 gauge needle. Straight lines were deposited with a write speed of $30 \mathrm{~mm} / \mathrm{sec}$ and a line pitch of $0.25 \mathrm{~mm}$. The deposition height was $100 \mu \mathrm{m}$ and dispense pressures were 10, 15, 20, 25, and 30 PSI. Layer thicknesses ranging from $25 \mu \mathrm{m}$ at $10 \mathrm{PSI}$, to $55 \mu \mathrm{m}$ at 30 PSI were achieved, with a dispense pressure of 25 PSI resulting in $50 \mu \mathrm{m}$ thick layers (Figure 5.12). It's important to note that the volume of material deposited at 10 PSI was insufficient to cause the individual beads to flow together, showing that a tighter pitch would be required for dispensing a continuous layer at this pressure with a 32 gauge needle. 
This experiment was repeated with a 21 gauge needle and a line pitch of $1 \mathrm{~mm}$ to achieve greater layer thicknesses. The deposition pressures used were 1.5, 2, 2.5, 3, and 3.5 PSI and layer thicknesses ranged from $50 \mu \mathrm{m}$ at 1.5 PSI to $225 \mu \mathrm{m}$ at 3.5 PSI (Figure 5.13). Again, it is important to note that the volume of material deposited at 1.5 PSI was insufficient to cause the individual beads to flow together, showing that a tighter pitch would be required for dispensing a continuous layer at this pressure with a 21 gauge needle.

These experiments demonstrated the ability to write controlled layer thickness ranging from 25-220 $\mu \mathrm{m}$ (Table 5.2) while significantly reducing the amount of material used for each sample. Because there was no excess material used, the need for solvents was greatly reduced.

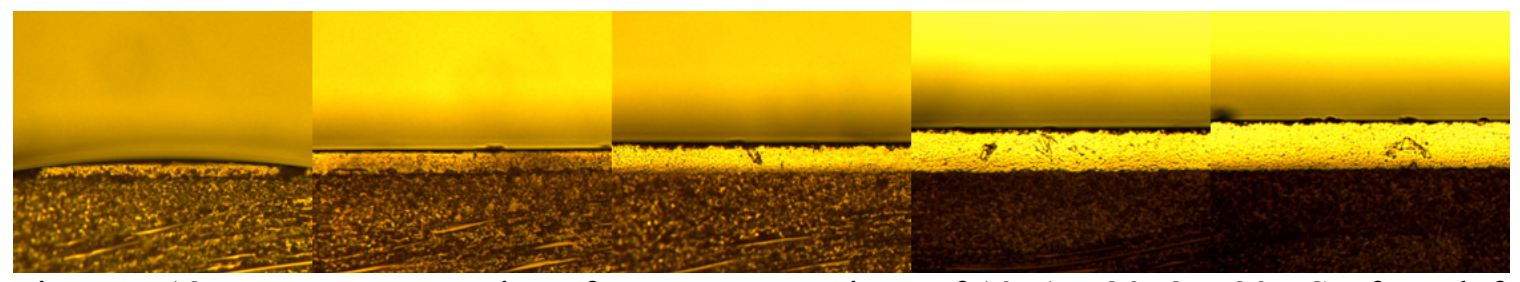

Figure 5.12. Layer cross sections for pressure settings of 10, 15, 20, 25, 30 PSI, from left to right. Thicknesses range from $25 \mu \mathrm{m}$ at 10 PSI to $55 \mu \mathrm{m}$ at 30 PSI for a line pitch of $0.25 \mathrm{~mm}$

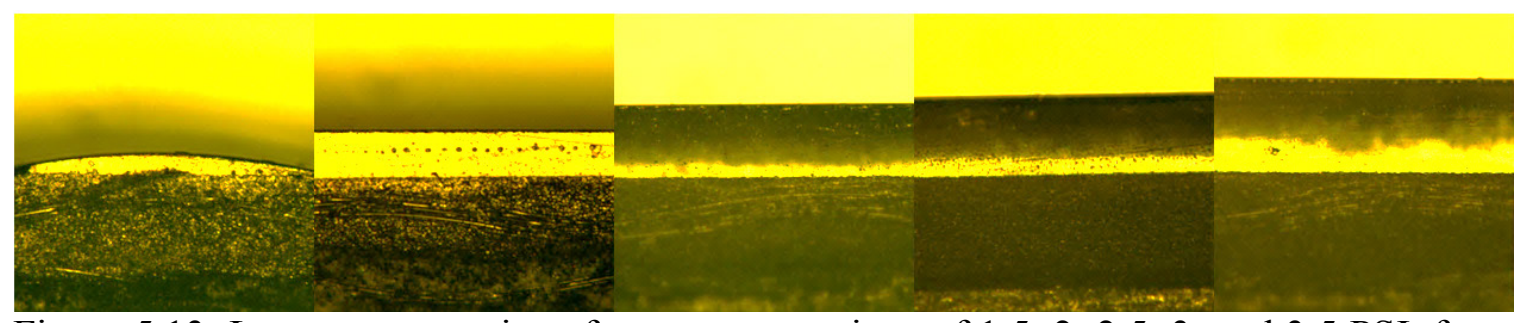

Figure 5.13. Layer cross sections for pressure settings of 1.5, 2, 2.5, 3, and 3.5 PSI, from left to right. Thicknesses range from $50 \mu \mathrm{m}$ at 1.5 PSI to $220 \mu \mathrm{m}$ at 3.5 PSI for a line pitch of $1 \mathrm{~mm}$.

Table 5.2

Layer thicknesses by dispense pressure for 21 and 32 gauge needle

\begin{tabular}{|c|c|}
\hline \multicolumn{2}{|c|}{21 Gauge Needle* } \\
\hline $\begin{array}{c}\text { Dispense } \\
\text { Pressure }\end{array}$ & $\begin{array}{c}\text { Layer } \\
\text { Thickness }\end{array}$ \\
\hline PSI & $\mu \mathrm{m}$ \\
\hline 1.5 & $51.72^{* *}$ \\
2 & 106.53
\end{tabular}

\begin{tabular}{|c|c|}
\hline \multicolumn{2}{|c|}{32 Gauge Needle* } \\
\hline $\begin{array}{c}\text { Dispense } \\
\text { Pressure }\end{array}$ & $\begin{array}{c}\text { Layer } \\
\text { Thickness }\end{array}$ \\
\hline PSI & $\mu \mathrm{m}$ \\
\hline 10 & $30.93^{* *}$ \\
15 & 26.91
\end{tabular}




\begin{tabular}{|c|c|c|c|}
\hline 2.5 & 173.03 & 20 & 34.02 \\
\hline 3 & 187.81 & 25 & 50.41 \\
\hline 3.5 & 219.21 & 30 & 56.91 \\
\hline \multicolumn{2}{|c|}{ *1.0 mm Line Pitch } & \multicolumn{2}{|c|}{$* 0.25 \mathrm{~mm}$ Line Pitch } \\
\hline \multicolumn{2}{|c|}{ **Beads Did Not Combine } & \multicolumn{2}{|c|}{ **Beads Did Not Combine } \\
\hline
\end{tabular}




\subsection{Dispensing Discrete Waveguides}

The next step in the characterization of the direct dispense process was to focus on the deposition of discrete waveguides which do not require any UV definition or development. The same process used for dispensing layers was followed, with the pitch being set to $1 \mathrm{~mm}$ to maintain separation between the individual waveguides. Waveguides were written using a 33 gauge needle at a write speed of $30 \mathrm{~mm} / \mathrm{sec}$ and a dispense height of $100 \mu \mathrm{m}$. Four different material blends were studied: 70, 75, 80, and $85 \%$ NVC core material.

To dispense the $70 \%$ NVC material, dispense pressures of $4,5,6,7,8,9,10,15,20,25$, 30, and 35 PSI were used. The dispense process behaved as expected, and similar to previous work, with higher pressures resulting in more volume being dispensed, resulting in larger waveguides. Through this method, continuous waveguide beads ranging from $625 \mu \mathrm{m}$ down to $150 \mu \mathrm{m}$ in width and $50 \mu \mathrm{m}$ down to $15 \mu \mathrm{m}$ in thickness (Figure 5.14) were produced. The $70 \%$ NVC waveguides had a semi-elliptical shape, where the width and thickness increased as a direct function of dispense pressure (Figure 5.15 and Figure 5.16).
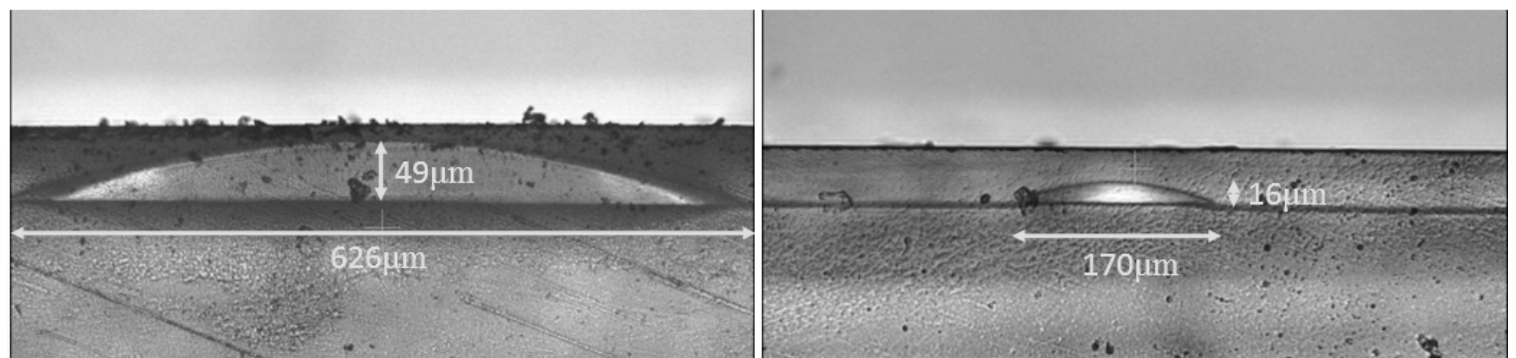

Figure 5.14. Guides written at 35 PSI (left) and 4 PSI (right) with a 32 gauge needle. 

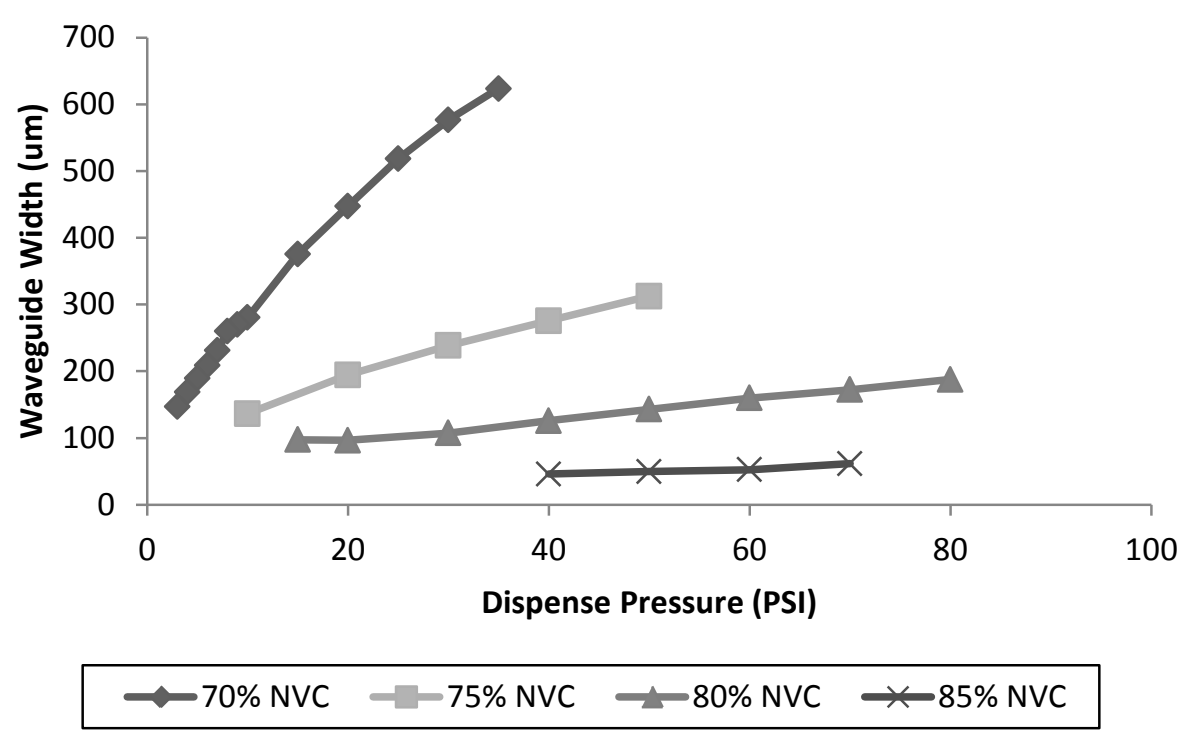

Figure 5.15. Waveguide width as a function of dispense pressure for $70,75,80$, and $85 \%$ NVC core material through a 33 gauge needle
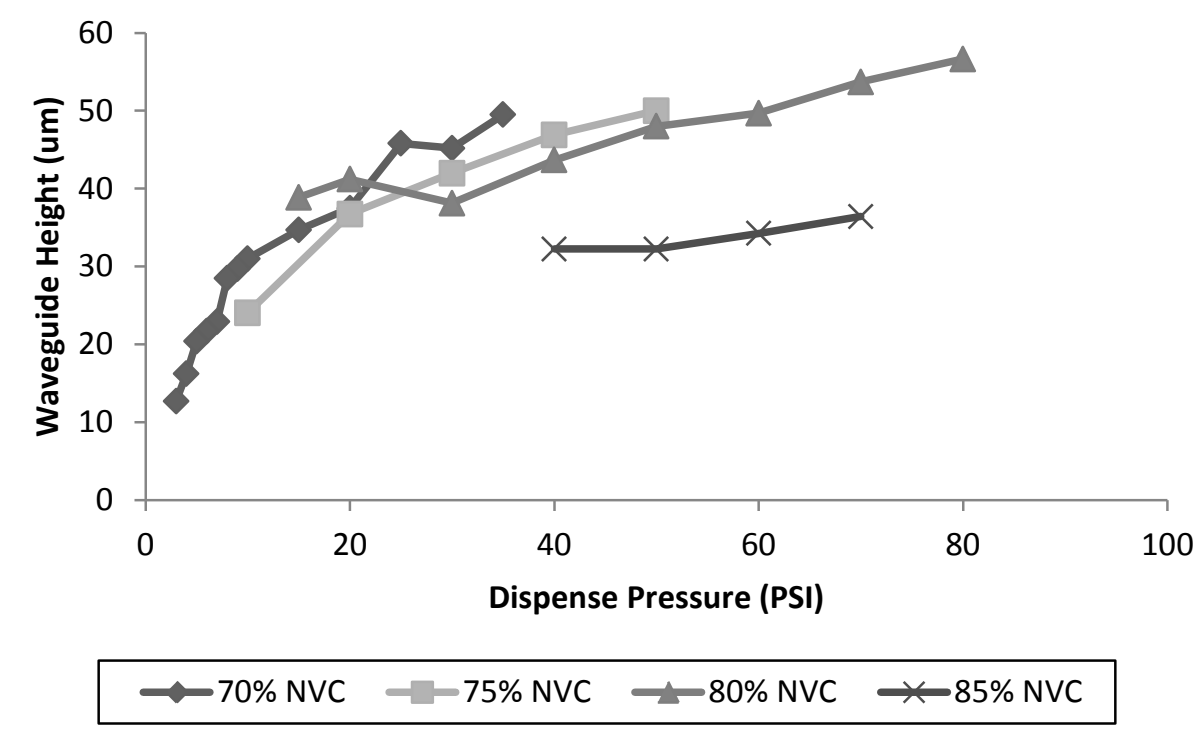

Figure 5.16. Waveguide height as a function of dispense pressure for $70,75,80$, and $85 \%$ NVC core material through a 33 gauge needle

The process was repeated for the $75 \%$ blend at dispense pressures of $10,20,30,40$, and 50 PSI. It was again seen that increased dispense pressures lead to larger waveguides. One noticeable difference was the increased contact angle of the beads with the increase in \%NVC. This resulted in higher aspect ratios and more semi-circular shapes, seen in Figure 5.17. 

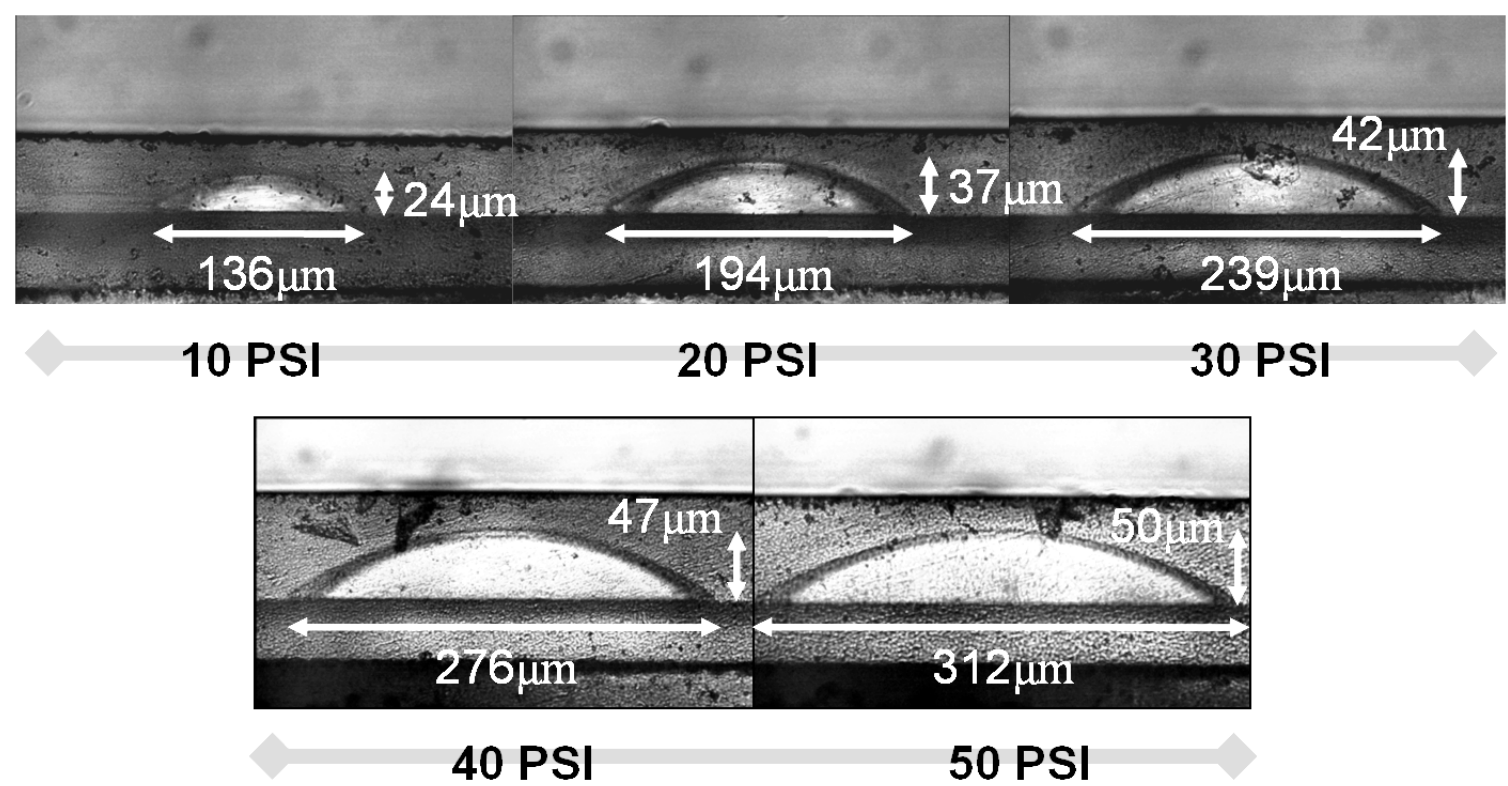

Figure 5.17. 75\% Solid content core material dispensed through a 33 gauge needle.

When the $80 \%$ blend was dispensed (at dispense pressures of $15,20,30,40,50,60,70$, and $80 \mathrm{PSI}$ ), the structures began to exhibit an even higher contact angle (Figure 5.18). It is hypothesized that the toluene present in the liquid core material binds well with the cured cladding material and by decreasing the amount of toluene present in the liquid core blend (increasing the \% NVC), the material becomes more hydrophobic when deposited onto cured cladding.

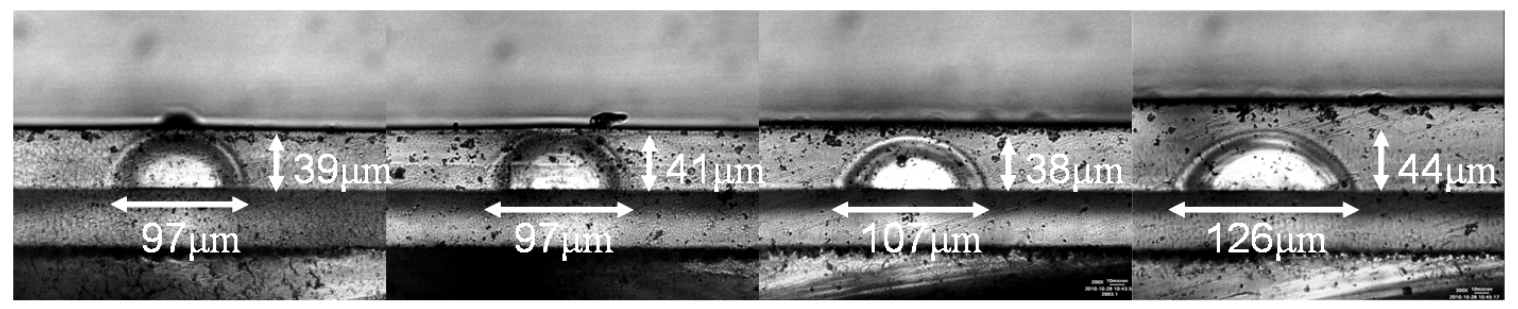
$15 \mathrm{PSI}$ $20 \mathrm{PSI}$ $30 \mathrm{PSI}$ $40 \mathrm{PSI}$

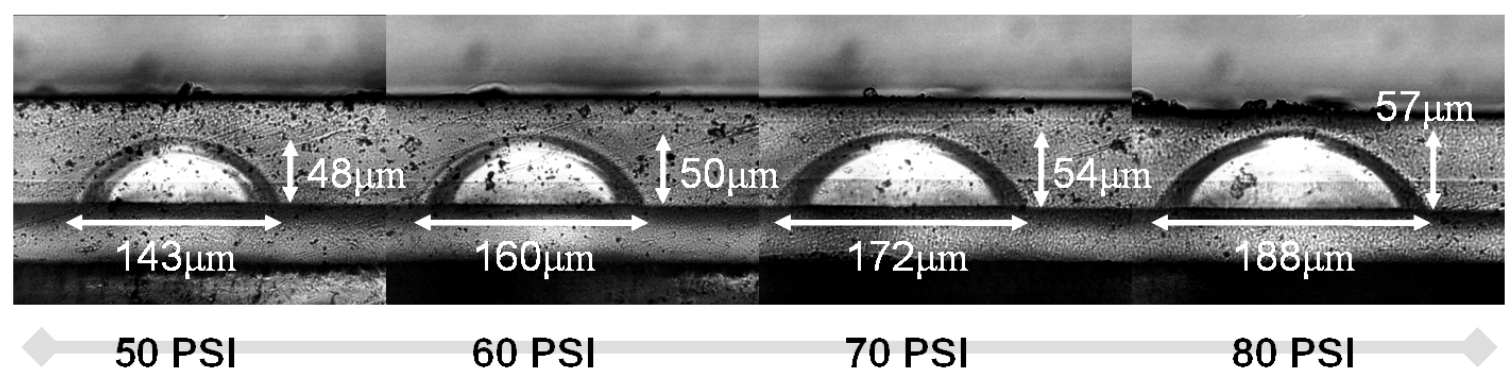

Figure 5.18. 80\% Solid content core material dispensed through a 33 gauge needle. 
The $85 \%$ NVC core material was dispensed at $40,50,60$, and 70 PSI and exhibited the greatest contact angle of all the blends, resulting in nearly round waveguide structures (Figure 5.19). Having less toluene in the blend kept the liquid material from "washing out" when dispensed, resulting in aspect ratios approaching 1:1 (Figure 5.20). The resulting guides from the $85 \%$ trial were tested and had an initial attenuation range of $0.06-0.09 \mathrm{~dB} / \mathrm{cm}$, which is comparable to lithographically fabricated square waveguides.

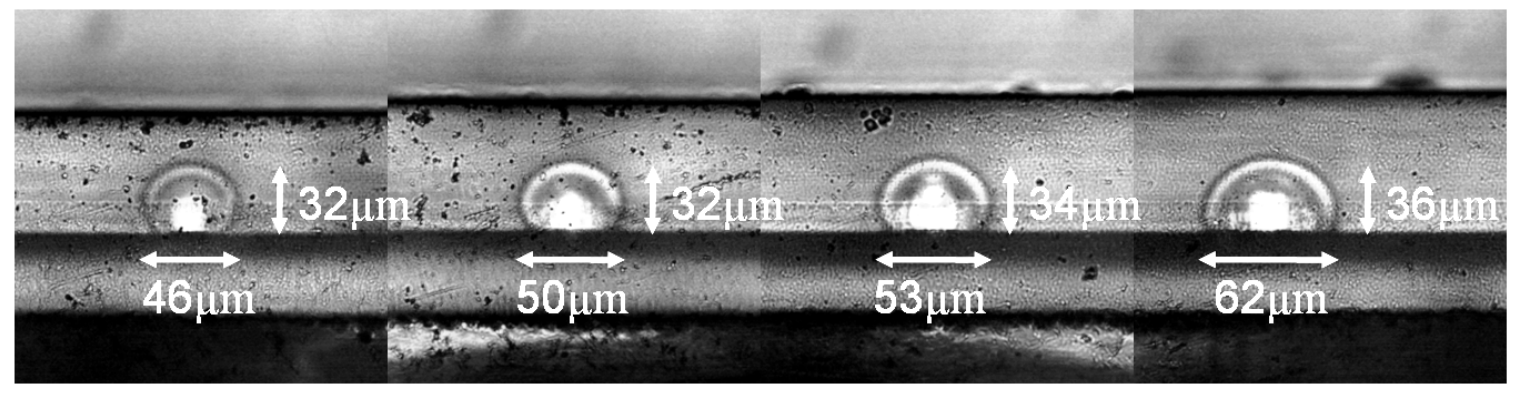

40 PSI 50 PSI $60 \mathrm{PSI}$ 70 PSI

Figure 5.19. 85\% Solid content core material dispensed through a 33 gauge needle.

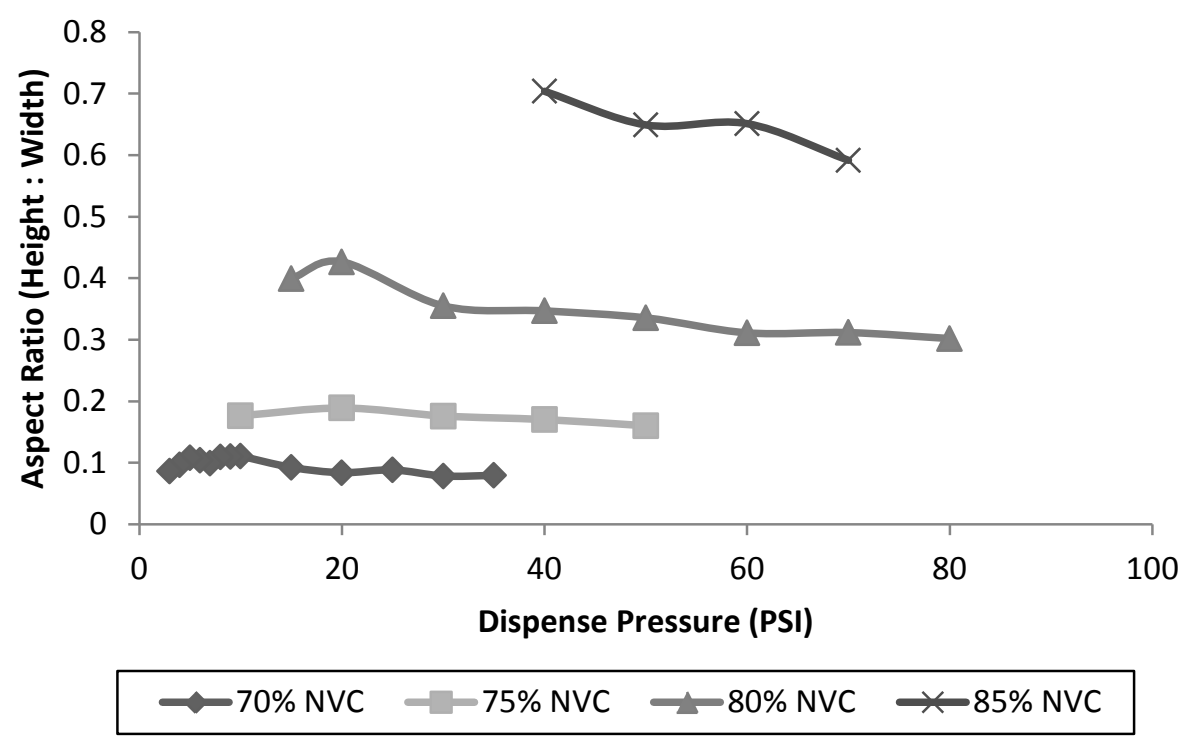

Figure 5.20. Waveguide aspect ratio (height : width) as a function of dispense pressure for $70,75,80$, and $85 \% \mathrm{NVC}$ core material through a 33 gauge needle.

The $85 \%$ material experiment was repeated at a lower dispense height of $25 \mu \mathrm{m}$ to see if smaller guides could be patterned. During experimentation, it was observed that a higher range of pressures $(55,65,75$ and $80 \mathrm{PSI})$ was required to establish continuous waveguide structures. Figure 5.21 shows the resulting cross sections of this experiment, noting that the guides written with 55 PSI did not survive further processing. By reducing the dispense height, the waveguides were reduced in size by about half. 

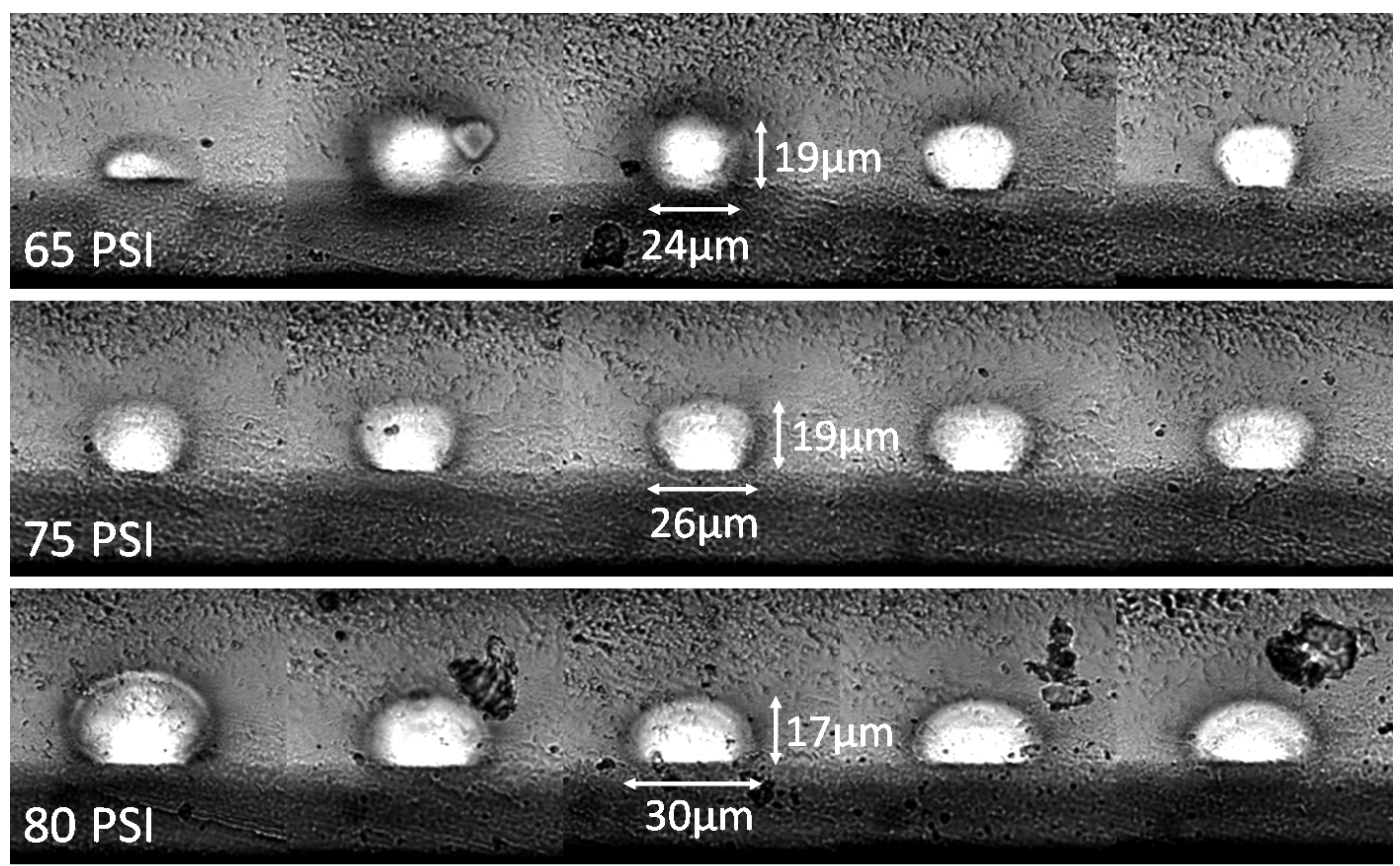

Figure 5.21. Direct dispense waveguide cross sections for pressures of 65, 75, 80 PSI.

\subsection{Direct Dispense Conclusions and Future Work}

A direct dispense tool was created and characterized for the repeatable dispensing of blanket waveguide layers over a range of thicknesses $(25-220 \mu \mathrm{m})$, eliminating waste material and affording the ability to utilize large substrates. This tool was also used to directly dispense multimode waveguide cores which required no UV definition or development. These cores had circular cross sections and were comparable in optical performance to square, lithographically fabricated waveguides. Initial write speeds of 30 $\mathrm{mm} / \mathrm{sec}$ were demonstrated with capabilities up to $100 \mathrm{~mm} / \mathrm{sec}$. A 33 gauge needle was successfully utilized with possibilities of commercially available dispense tips with submicron inner diameters for even smaller features. The dispensed features were already seen to be smaller than that of the inner diameter of the dispense needle. Four material blends were evaluated with the contact angle of the waveguides increasing with increased $\% \mathrm{NVC}$, resulting in higher aspect ratios. The round shape of the guides allows for greater coupling tolerance using a round core input or output fiber.

Dispense height proved to be a significant obstacle in the dispense process, as small variances can result in discontinuous waveguides. Future work would include investigating methods of maintaining a constant dispense height during the direct write process. A possible solution to this problem is the inclusion of a guide pin, or needle foot, that extends a fixed distance beyond the needle tip, and is meant to ride along the surface of the substrate (Figure 5.22). This pin would be directly integrated with the dispensing needle. Mounted into a spring loaded fixture, the syringe assembly would 
"float" a fixed distance above the sample surface, allowing the dispensing height to remain constant over uneven surface topology. Even though it is a contact process, this dispensing set up would make holding a dispensing height over an entire board repeatable. A non-contact solution would be to integrate a surface scanning metrology tool capable of providing topological feedback to the controller for in-process adjustment of the Z-axis to match the contour of the substrate.

Future work would also include examining the effects of substrate and material deposition temperature on resulting layers or guides. Heating core material may result in smoother features and even higher aspect ratios as toluene is evaporated from the uncured material (Variation in the line stability of an inkjet printed optical waveguide applicable material, 2008).

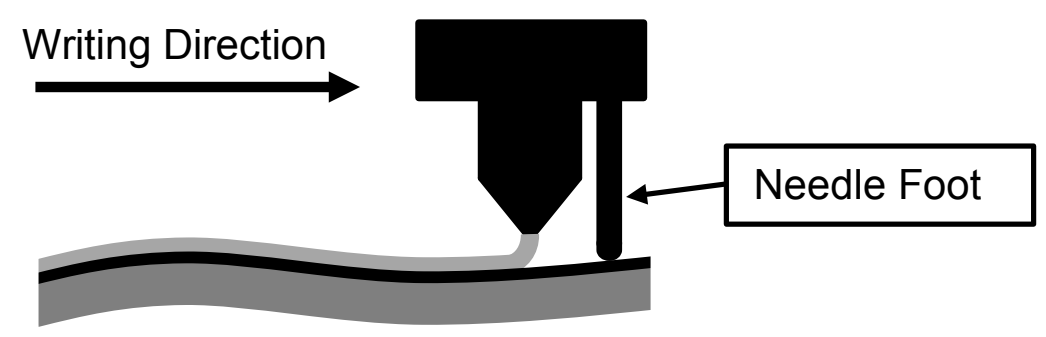

Figure 5.22. Shows the dispense needle and guide pin configuration. 


\section{Chapter 6. Optical Electrical Printed Wiring}

\section{Board Fabrication}

The development of the direct waveguide fabrication tool was completed with the goal of creating fully functional optical electrical printed wiring boards for military applications. Having demonstrated the ability to reliably fabricate and test waveguides, the challenge was to combine electrical and optical circuit board layers, and develop connectors for interfacing with these layers. To reduce costs and increase the chance of success, it was important to leverage existing commercial technology to develop a method of turning the optical signal out of the board plane (optical vias) with a means of coupling it into a style of connector common to the telecommunication industry. These components had to be rugged enough to withstand extreme environmental conditions (thermal, shock, humidity) in order to meet current military specifications. The integrated board level optical vias, or light turning devices (LTDs) enable non-planar routing of optical signals around, into, and out of the optical layer of the printed wiring board, illustrated in Figure 6.1. Optical vias have been studied in the past and can be formed through a variety of methods, which include etching, micro-embossing with heat, angled exposure, micro-molding, laser ablation, dicing and polishing. These methods have all been successful in producing optical vias in a laboratory environment, but one of the barriers to implementing this technology in a military setting is the lack of a mil-qualified board-level optical via. Other groups (IBM, Vario Optics, Xyratex) have successfully integrated optical vias to create manufacturable optical electrical printed wiring boards with total optical waveguide interconnect losses ranging from $6 \mathrm{~dB}$ to $13 \mathrm{~dB}$, but these were developed for commercial data and telecommunications applications and have not been proven to withstand extreme conditions $(7,70-72)$. The requirements for the design of the monolithic integrated optical via were that it have an efficient coupling interface (1-3 dB optical loss per interface) with low modal distortion, was cost effective, and was able to withstand manufacturing processes common to printed circuit boards as well as the extreme environments seen in military applications, such as extreme temperature and humidity.

\subsection{Design Rules}

Having characterized the essential waveguide structures (straights, turns), the performance data helped guide the requirements of the remaining components necessary for the construction of an optical electrical printed wiring board. The optical pathway being evaluated included the cabling and connectors used to send and receive signals external to the board, the light turning devices which route the signal into the optical layer of the board, and the waveguides that make up the optical layer, illustrated in Figure 
6.1. Knowing the signal loss associated with the various components provided a target loss threshold for the design and implementation of the light turning devices (Table 6.1). The performance metric that was monitored was output power, or total optical loss of the system on the $\mathrm{dB}$ scale, which was not to exceed $10 \mathrm{~dB}$. This included all cabling and connections from the laser source to the power head where the measurements were taken. It was also important that the device meet certain environmental requirements, primarily that the device withstands and remains operational over a temperature range of $-40^{\circ} \mathrm{C}$ to $110^{\circ} \mathrm{C}$.

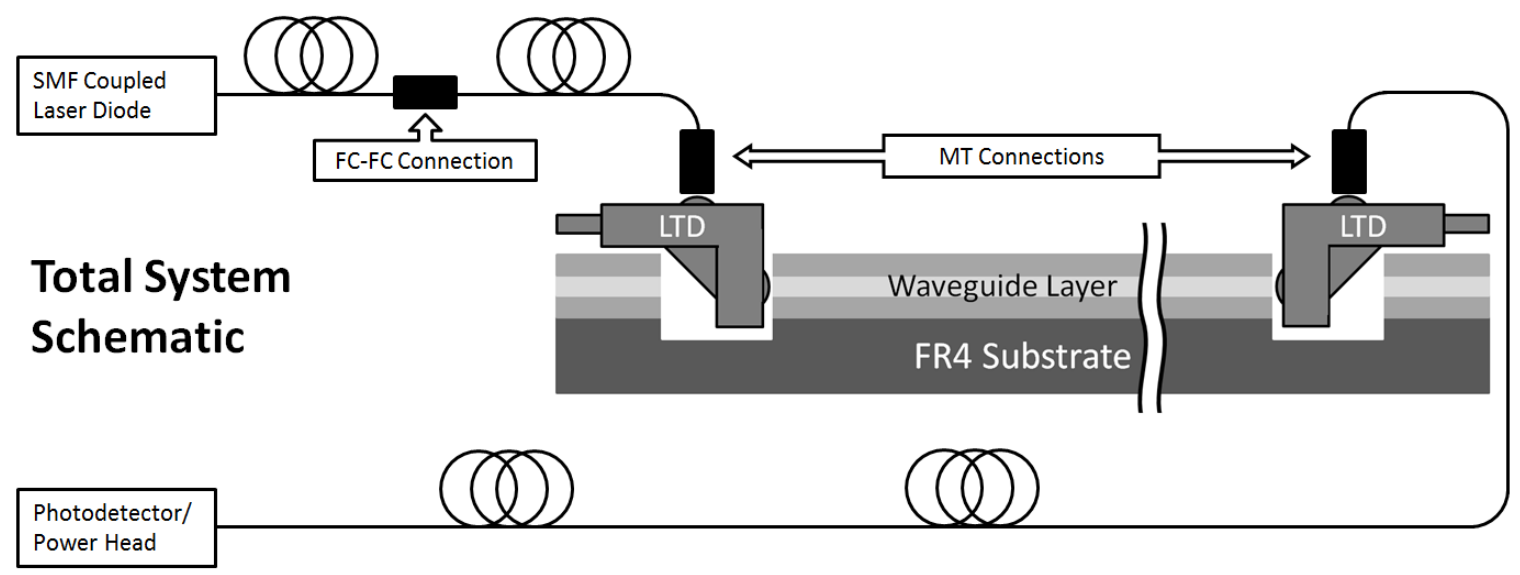

Figure 6.1. Optical routing schematic used for performance testing at Michigan Technological University.

Table 6.1

Power loss link budget, budgeting acceptable losses for specific design areas to ensure total losses do not exceed the $10 \mathrm{~dB}$ limit.

\begin{tabular}{|l|c|}
\hline Component & dB Loss \\
\hline Power Budget & $10 \mathrm{~dB}$ \\
\hline Component & dB Loss \\
\hline Two Optical Vias (LTD) $(2 * 2 \mathrm{~dB} /$ Via) & $4 \mathrm{~dB}$ \\
LTD Alignment $(2 * 1 \mathrm{~dB} / \mathrm{LTD})$ & $2 \mathrm{~dB}$ \\
MT Connector $(2 * 0.25 \mathrm{~dB} /$ connector) & $0.5 \mathrm{~dB}$ \\
MM Waveguide at $850 \mathrm{~nm}(10 \mathrm{~cm} * 0.06 \mathrm{~dB} / \mathrm{cm})$ & $0.6 \mathrm{~dB}$ \\
Optical Bend (4 turns at $0.25 \mathrm{~dB} /$ bend $)$ & $1 \mathrm{~dB}$ \\
Dispersion Margin & $1 \mathrm{~dB}$ \\
\hline Total Span Loss & $\mathbf{9 . 1} \mathbf{~ d B}$ \\
\hline *Power Margin $\left(\mathbf{P}_{\mathbf{M}}\right)=$ Power Budget $\left(\mathbf{P}_{\mathbf{B}}\right)$-Span Loss $\left(\mathbf{P}_{\mathbf{S}}\right)$ & $\mathbf{0 . 9} \mathbf{~ d B}$ \\
\hline
\end{tabular}




\subsection{Overview of Components}

The first two components of the OEPWB prototype were the individual optical and electrical board layers. The optical layer, which contained the waveguide routing, was combined with a separate electrical layer to form the printed wiring board stack. These layers were fabricated independently and joined together after component population. Added to the optical layer were pairs of light turning devices to route the signal into, and out of, the waveguides. The last major component of the OEPWB prototype were protective shrouds or receivers added to shield the LTDs from contamination after insertion, and also to secure the connectors for the input and output fiber optic cables during handling and testing to remove stress on the LTDs.

\subsection{Printed Wiring Board}

\subsubsection{Electrical Layer}

The $100 \mathrm{~mm}$ square FR4 electrical layer was minimal in design, as the production of electrical printed wiring boards has long been an established practice, requiring no further investigation. The simple electrical layer was added to provide a realistic form factor to the prototype to gain a firm practical understanding of how the electrical and optical layers would be joined together, and to provide an accurate physical representation during subsequent environmental testing. The design prints for the electrical layer, seen in Figure 6.2, show eight sets of electrical bond pad arrays in the center portion of the sample, and eight cutout windows toward the perimeter to accommodate the optical components when layered over the optical layer. Figure 6.3 shows how the two layers interface, with the full OEPWB assembly shown, complete with electrical components. Around the perimeter of the electrical layer are six pin holes for alignment between the board layers, as well as with the fixtures used during fabrication and testing.

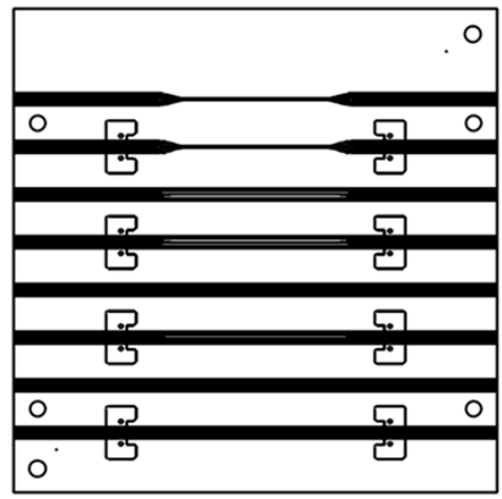

(A)

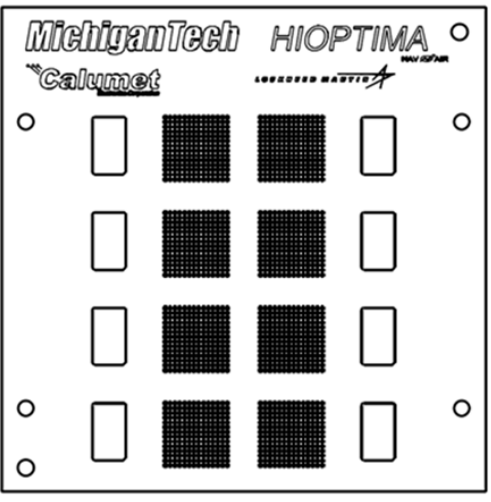

(B)

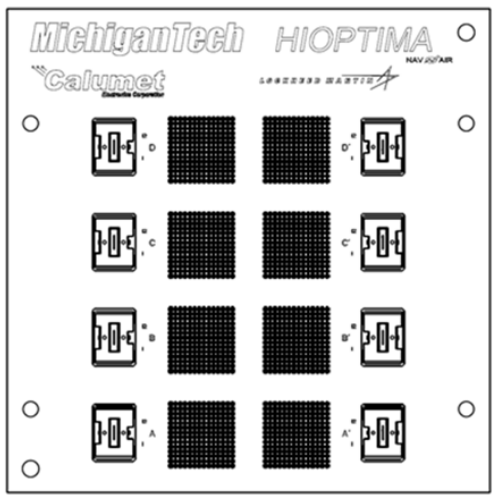

(C)

Figure 6.2. (A) Optical layer with machined LTD insertion pockets (B) Electrical overlay panel (C) Complete optical assembly with electrical overlay panel. 


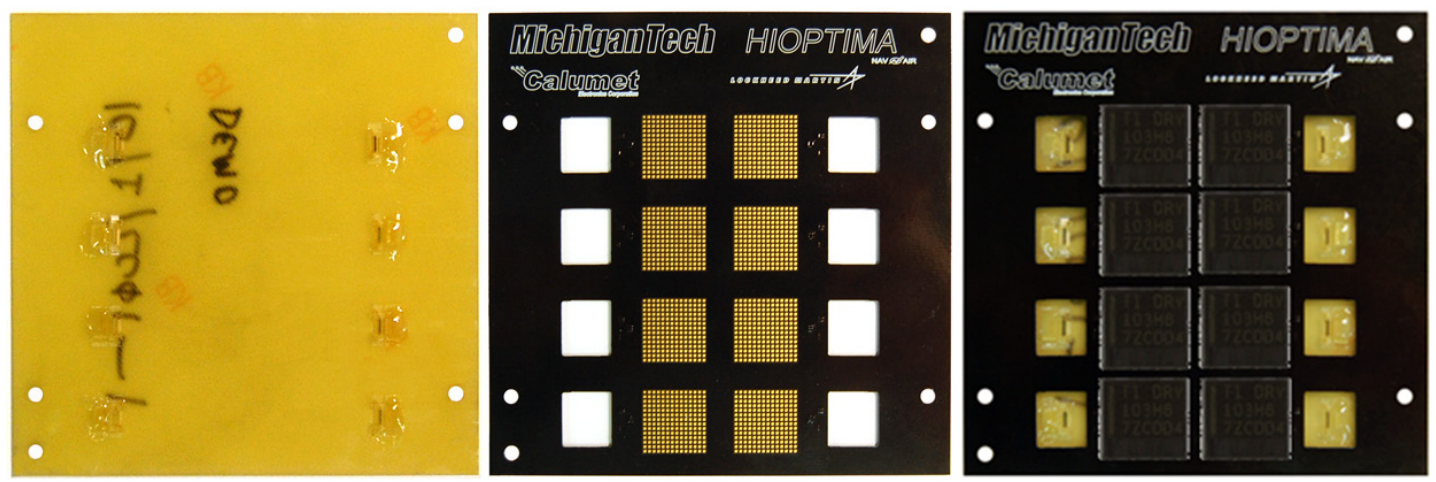

Figure 6.3. (A) Optical layer with generic optical components (B) Electrical layer overlay (C) Complete optical assembly with electrical overlay panel.

\subsubsection{Optical Layer}

The $100 \mathrm{~mm}$ square FR4 optical layer was designed to mate with the electrical layer using matching pinholes for layer alignment. The optical routing pattern consisted of four individual waveguide sets, duplicated for a total of eight sets. The duplicates remained unmodified and served as a control for comparing against their connectorized counterparts, seen in Figure 6.2(A). Each set contained 12 waveguides, the ends of which were spaced at a center-to-center pitch of $250 \mu \mathrm{m}$ to match standard MT cabling. The waveguide pitch was decreased in the center third of the sample for three of the four waveguide sets, seen in Figure 6.2(A) and detailed in Figure 6.4. The tighter pitch value was $100 \mu \mathrm{m}$, leaving $50 \mu \mathrm{m}$ of space between each of the $50 \mu \mathrm{m}$ waveguides.

(A)

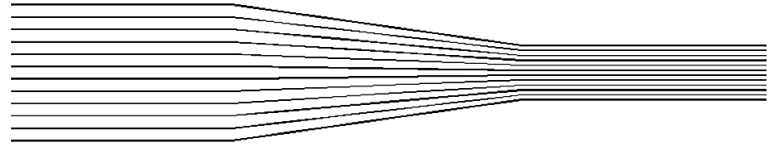

(B)

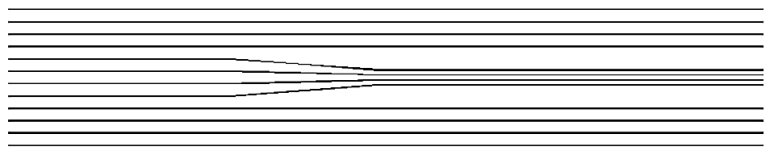

(C)

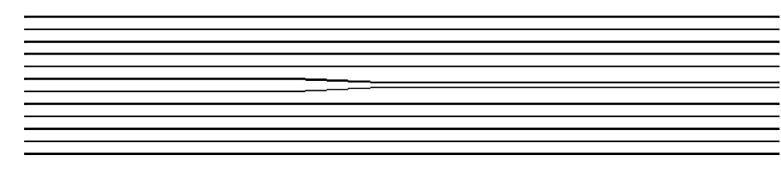

(D)

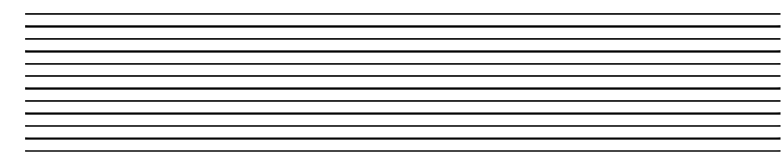

Figure 6.4. CAD detail of four patterned waveguide configurations for optical layer examining effect of waveguide pitch on optical performance: (A) Pitch narrows from $250 \mu \mathrm{m}$ to $100 \mu \mathrm{m}$ for all 12 waveguides (B) Pitch narrows from $250 \mu \mathrm{m}$ to $100 \mu \mathrm{m}$ for center four waveguides (C) Pitch narrows from $250 \mu \mathrm{m}$ to $100 \mu \mathrm{m}$ for center two waveguides (D) Pitch remains at standard $250 \mu \mathrm{m}$ for all 12 waveguides. 


\subsection{Optical Interconnect}

\subsubsection{Light Turning Device}

To turn the light out of plane and route signals between the optical layer and the external cabling, a commercially available light turning device (LTD) from microPEP (East Providence, RI) was utilized. The microPEP component, seen in Figure 6.5, is a 12channel, right angle coupler, injection molded from Ultem, a material capable of operating over a wide range of temperatures $\left(-40^{\circ}\right.$ to $\left.+150^{\circ} \mathrm{C}\right)$. It features a total internal reflection mirror surface to turn the light signal $90^{\circ}$ and incorporates lenses on both sides of the turn to reduce coupling loss. The design includes pin holes based on existing MT pin alignment technology, making it easy to align external MT style connectors to the LTD. Though the intended purpose for this component was to mate fibers with vertical cavity surface emitting laser arrays at a right angle, it was successfully adapted to couple external MT-style cabling to embedded waveguides. This new component offered excellent performance through reduced surface roughness on the optical features, exhibiting a loss of $1.4 \mathrm{~dB}$ per $90^{\circ}$ turn. Because the part was currently being manufactured in quantity, the consistency of quality between parts was very good, essential for repeatability in OEPWB pilot production.
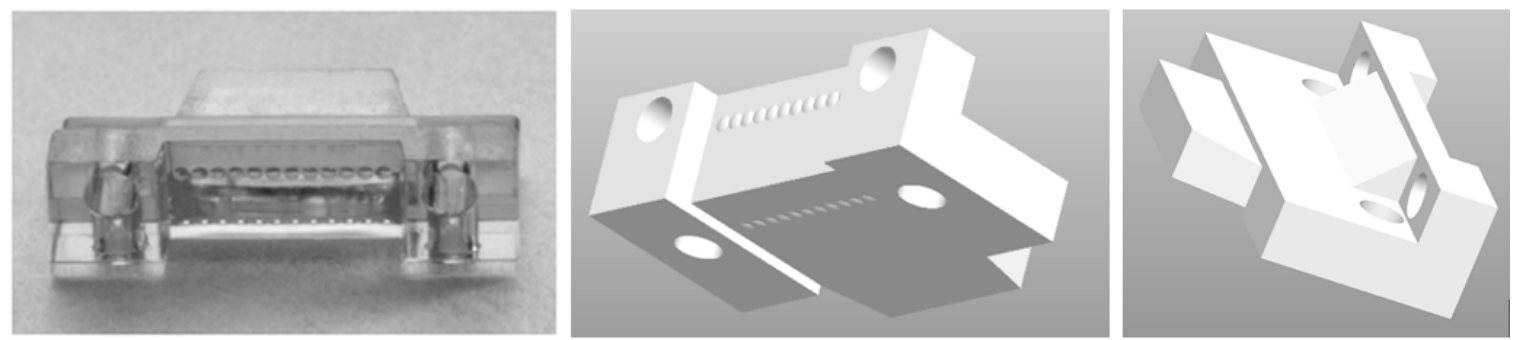

Figure 6.5. 12-Channel Micro Lens Array by microPEP (left) device (right) CAD representation.

\subsubsection{Connector Receiver}

The microPEP LTD made use of free space optics for containing and turning the light source, making surface contamination of these components a significant threat to overall performance. Any debris or film on the lenses or reflector face, due to manufacturing and general handling, or due to environmental exposure could render the LTDs inoperable. To address this, protective receivers were machined out of polycarbonate, a material capable of handling the required temperature extremes of $-40^{\circ} \mathrm{C}$ to $110^{\circ} \mathrm{C}$. The initial design, seen in Figure 6.6, was an intentionally simple concept designed to interface with a standard MT connector type and it addressed the primary purpose of protecting the embedded LTD from the ambient environment. 


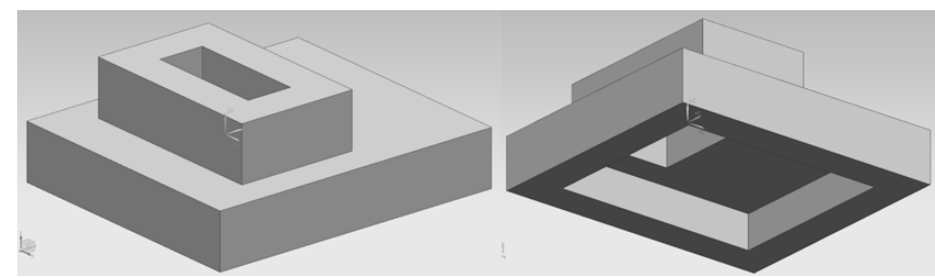

Figure 6.6. CAD design of basic MT style connector receiver. The larger cavity accommodates the LTD, which protrudes slightly from the board surface, and the smaller slot accommodates the external MT connector.

While other receiver types were developed, this was the style that was used for environmental testing of the OEPWB. The receivers effectively secured the external cabling and maintained alignment between the MT connectors and the embedded LTDs. Figure 6.7 shows how the receiver aided in relieving any strain imposed on the LTD by the external cabling during handling. It was also demonstrated that these receivers were very effective in protecting the LTD cavity from high moisture levels during humidity studies. One drawback of this design was that it had no method of "clipping" or otherwise securing the cabling if it were to undergo any kind of intense vibration. Another limitation was form factor, as it required that the cabling be routed vertically with respect to the board, causing it to have a much taller profile than desired.

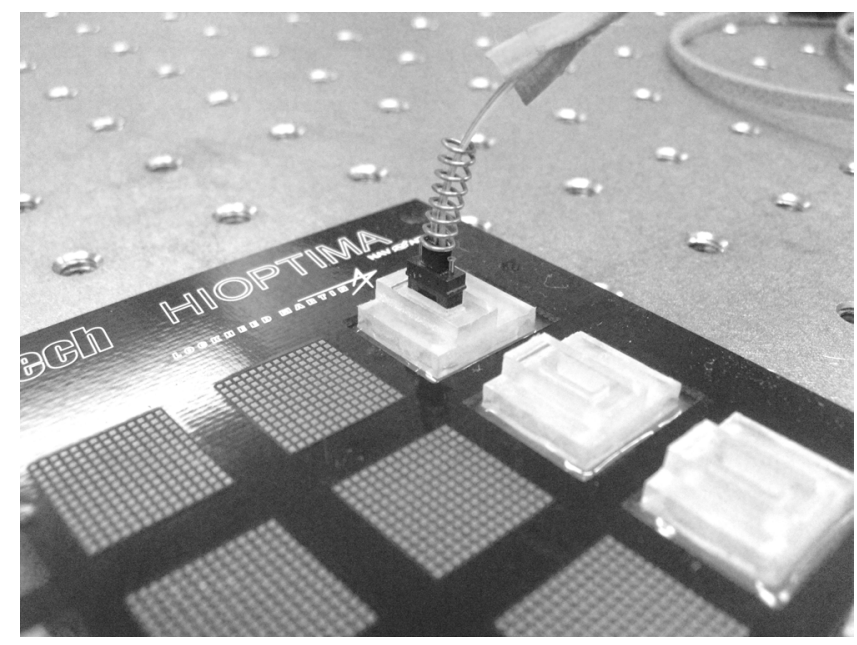

Figure 6.7. MT connector receivers provide environmental protection and stability to the optical device area.

One alternate design that was developed addressed the issue of securing the cabling for shock and vibration applications. This design still had the same form factor restriction as before (vertical cable routing), but it made great gains in addressing the issue of securing the incoming cable connector, and allowing it to resist failure as a result of vibration. To accomplish this, a commercially available MTP/MPO adaptor housing was adapted to act as the receiving connector on the board (Figure 6.8). It still protected the embedded LTD 
while securing the external cabling with its internal clips. It also made use of the internal compression system within the MTP/MPO connector to maintain constant contact between the cabling component and the LTD. A useful connector option available features a spring loaded shutter flap, which would seal the LTD cavity while the optical channel is not in use (Figure 6.8). This design was successfully fabricated in a laboratory environment, but did not undergo environmental testing.

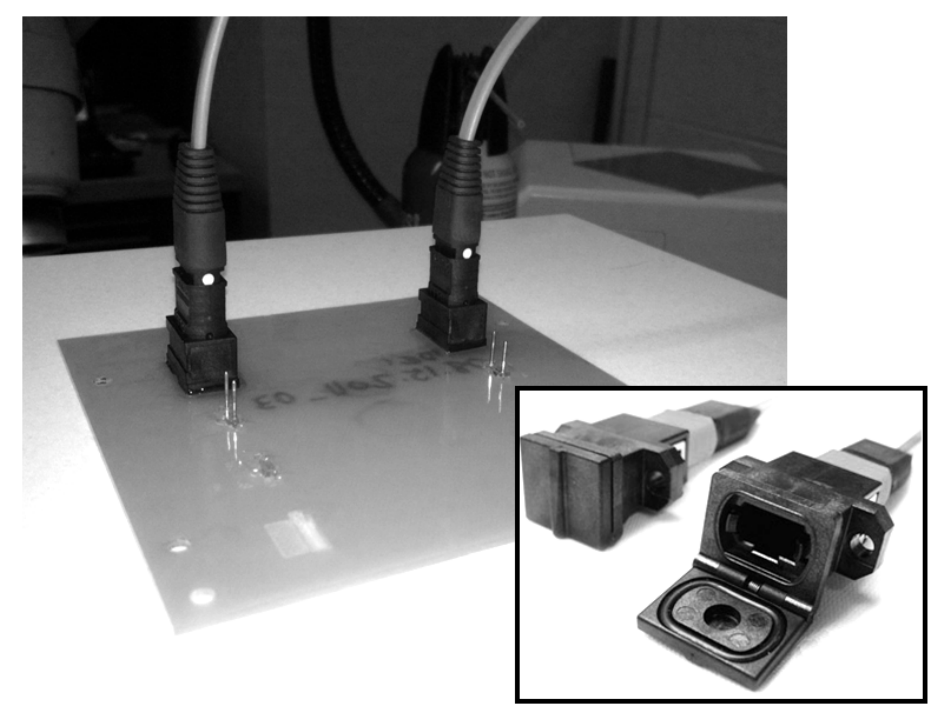

Figure 6.8. MTP/MPO style connection receptacles provide further environmental protection, as well as a more standardized form factor. Spring loaded compression fitting with clipping ability better suited for vibration testing.

Another alternate design, which was prototyped but not fully implemented, addressed the issue of form factor. It was designed to couple with a PRIZM connector from USCONEC (Hickory, NC), which would allow the external cabling to run parallel to the board, rather than orthogonally. This would yield a much improved form factor, while also addressing the issue of failure due to vibration, as the PRIZM component boasts a latch mechanism to secure the connector in place.

This modified receiver included design features which would limit the flow of epoxy around the free space optics located on the LTDs, a challenge encountered during the installation of the LTDs into the optical layer. These four "shoulder" protrusions, detailed in Figure 6.9, would help secure the part and would create a pocket area at each end for epoxy to be applied (Figure 6.10). Keeping the epoxy away from the LTD optics would limit a significant failure mode associated with the installation of the LTDs, resulting in a more reliable connection. The result would be a unified part, which would help mitigate potential board failure due to movement between independent parts. The unified component would then be implanted into the board as one piece. 


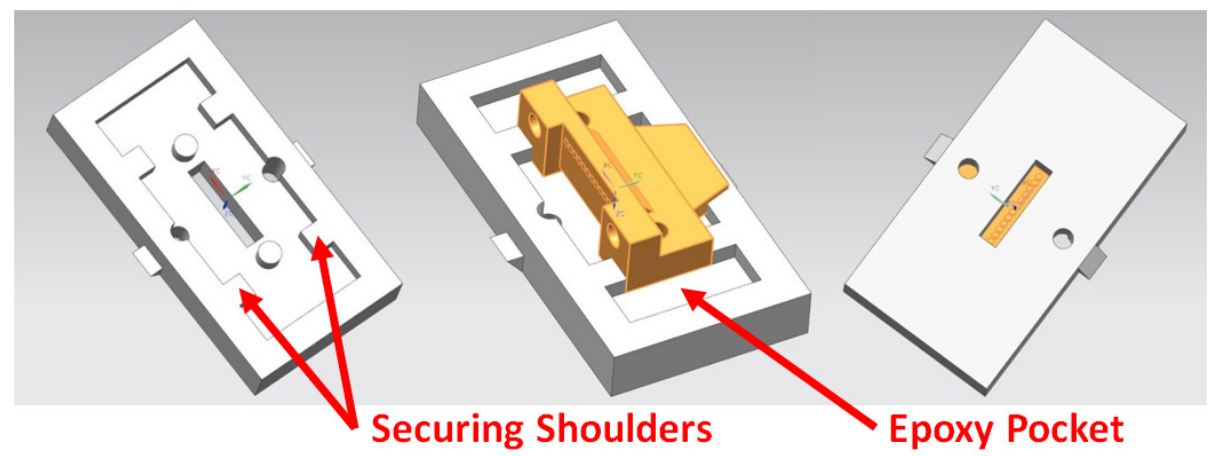

Figure 6.9. Illustrating how the LTD would snap into the modified receptacle using built in alignment features, and would then be epoxied into place at each end, away from the free space optics.

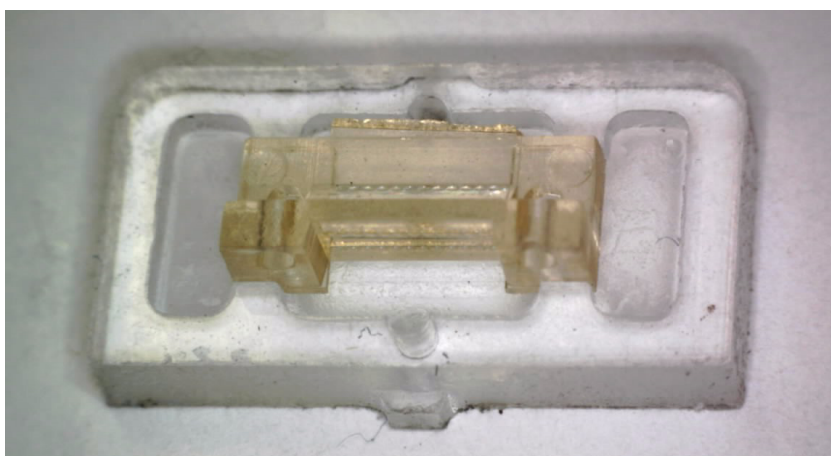

Figure 6.10. Machined receptacle with inserted LTD. Back "gate" portion had to be cut off of stock LTD to fit the receptacle.

This receiver featured built-in alignment holes specific to the PRIZM connector, and protrusions designed to mate with the locking clips of the PRIZM connector housing, seen in Figure 6.11. This would aid in reliable passive alignment, and result in a secure connection between the input and output cabling and the optical board. This design would be more suitable for an environment in which vibration is a concern, such as the current avionics focus.

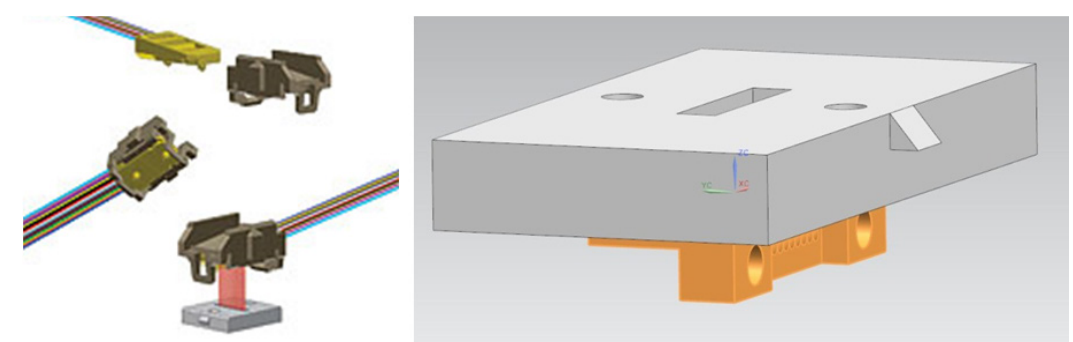

Figure 6.11. Alternate receiver design for mating with USCONEC PRIZM connector. This style offers improved form factor and ability to secure cabling for shock and vibration testing. 


\subsection{Fabrication and Assembly}

Implanting the LTD and receiver components offered the most significant challenge in developing a working prototype device, requiring that alignment be maintained between several process steps taking place on separate tools. This made sample setup and registration a key component for successful fabrication, often requiring special sample jigs or fixtures to ensure accuracy and repeatability. The first step in the construction process of the OEPWB, detailed in Figure 6.12, was to lithographically pattern the optical layer (this includes one core and two cladding layers) on an oversized (150 mm diameter circle, $1.5 \mathrm{~mm}$ thick) FR4 substrate. After the optical board layer was fabricated, it was machined to size $(100 \mathrm{~mm} \times 100 \mathrm{~mm}$ square) and alignment holes were drilled to accommodate $3.175 \mathrm{~mm}$ diameter pins. The machining work was performed on a Protomat S100 Rapid Circuit Board Plotter by LPKF, which boasts a high performance spindle capable of 100,000 RPM and system resolution of $0.25 \mu \mathrm{m}$ laterally, and $0.5 \mu \mathrm{m}$ vertically. After machining the sample to final form, the S100 was then used to machine $0.75 \mathrm{~mm}$ deep LTD insertion pockets into the optical layer at eight locations towards the perimeter of the sample, seen in Figure 6.12.

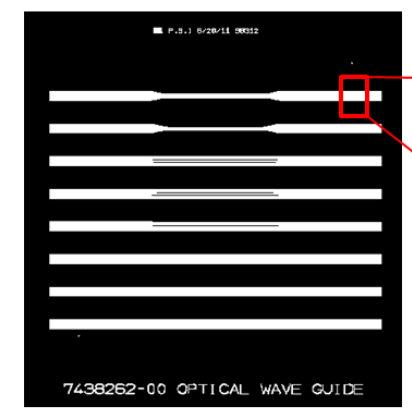

1) Lithographically Pattern Waveguides

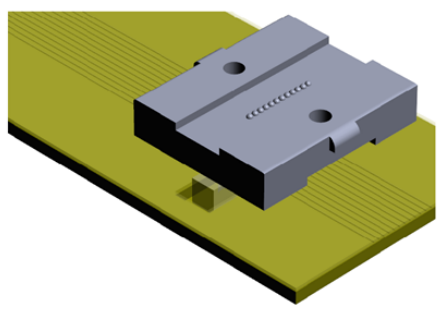

4) Attach Receiver

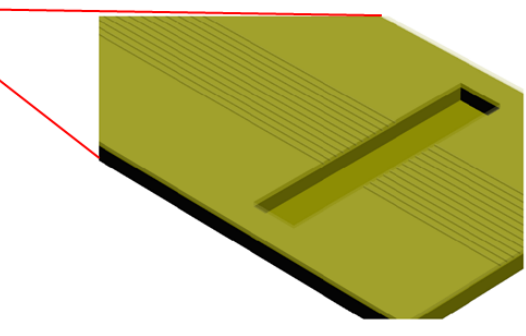

2) Mill Insertion Pocket

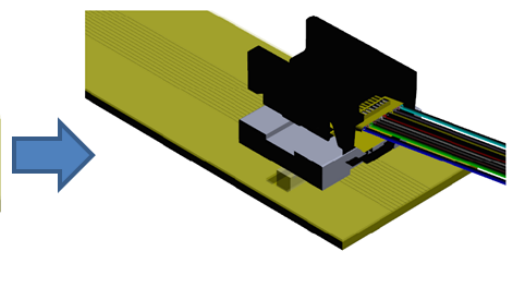

5) Attach Cabling

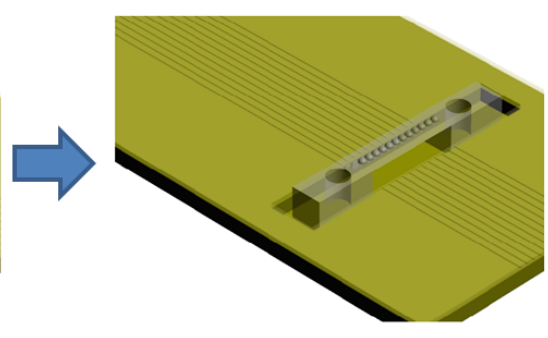

3) Insert LTD

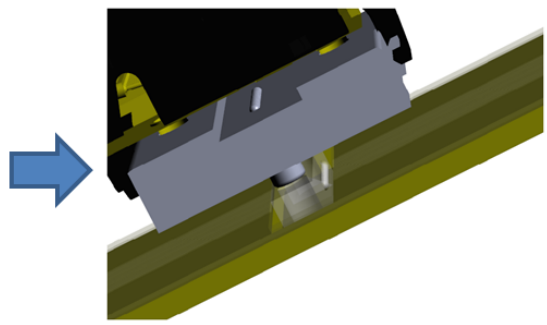

6) Test and Evaluate

Figure 6.12. OEPWB fabrication and assembly process.

Characterization work yielded information critical to achieving higher quality surface finishes at the waveguide end faces. When machining the insertion pockets, a $0.8 \mathrm{~mm}$ square carbide end mill was used at a feed rate of $10 \mathrm{~mm} / \mathrm{sec}$ and a spindle speed of $80,000 \mathrm{RPM}$. Using small bits $(0.8 \mathrm{~mm}$ diameter square end mill) improved surface 
finish, as the corner radius of the milling tool is smaller for smaller diameter bits, providing a sharper cutting edge. Slower feed rates and higher spindle speeds improved the finish of the cut, but it was observed that if feed rates were too slow, the sample would burn at such high spindle speeds. The most important observation was the impact of feed direction, or up milling as opposed to down milling, illustrated in Figure 6.13. The siloxane material is brittle when cured, and is prone to chipping and fragmenting when put in tension, as is the case when up milling. Putting the material in compression by down milling resulted in a more consistent surface finish which did not exhibit the same chipping and cracking, seen in Figure 6.14.

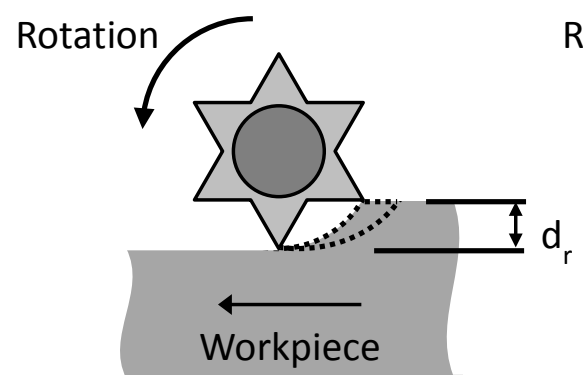

(A)

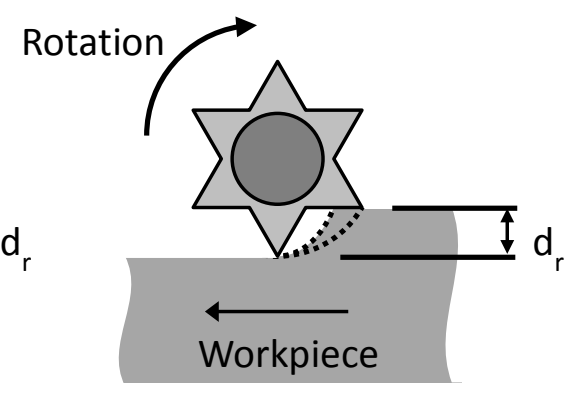

(B)

Figure 6.13. (A) Up milling puts the brittle waveguide material in tension, causing it to chip and fragment, drastically degrading optical quality (B) Down milling puts the brittle waveguide material into compression, reducing the amount of fragmentation resulting in a more uniform surface finish, where $d_{r}$ is radial depth of cut.

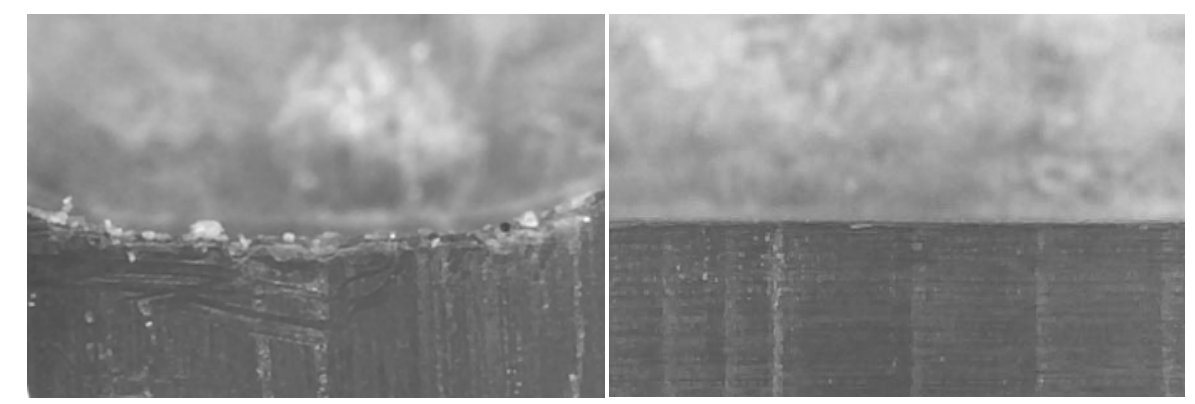

Figure 6.14. Siloxane material exhibits chipping when put in tension, due to improper feed direction (left) and improved cut quality when material is put in compression while down milling (right).

After the initial pockets were milled, a series of six "clean-up" cuts were made at decreasing radial depths of cut $\left(\mathrm{d}_{\mathrm{r}}\right)(250 \mu \mathrm{m}, 250 \mu \mathrm{m}, 100 \mu \mathrm{m}, 50 \mu \mathrm{m}, 25 \mu \mathrm{m}, 10 \mu \mathrm{m})$ to remove any chipped siloxane material and clean up the waveguide end (Figure 6.15). The waveguide end faces were then hand polished using 800 grit, followed by 1200 grit sandpaper. It was observed that the inclusion of this polishing process step led to significant increases in performance results, as increases in surface roughness at the 
waveguide end-face lead to greater scattering losses (Optical waveguide end roughness in correlation to optical coupling, 2012).

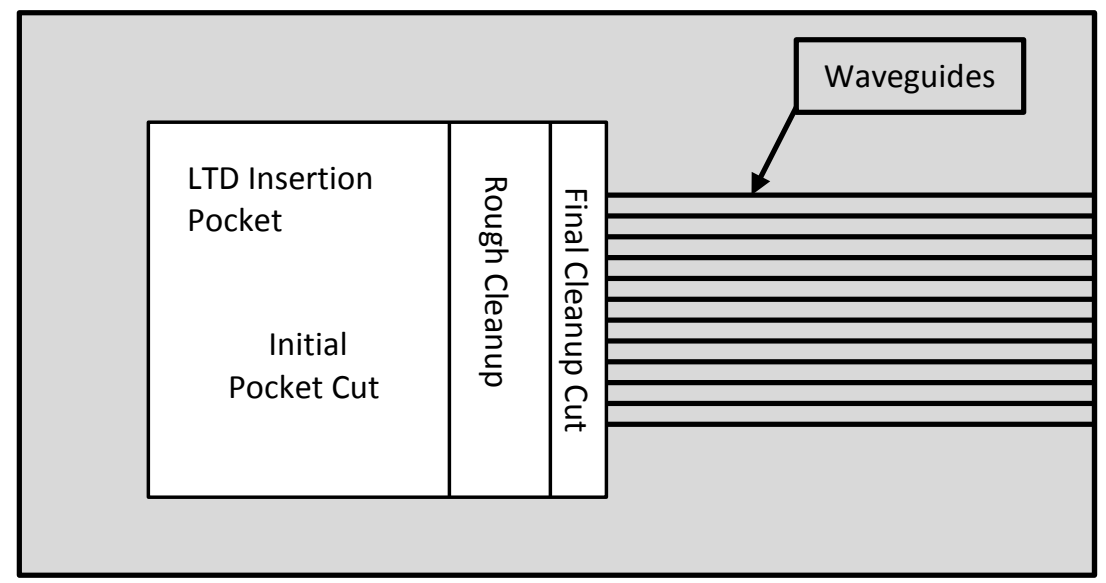

Figure 6.15. Milling schematic illustrating the inclusion of finish cuts at decreasing radial depths of cut $\left(\mathrm{d}_{\mathrm{r}}\right)$ to improve surface finish.

After the insertion pockets were machined and polished, the LTDs were inserted using precision optical alignment stages, capable of adjusting all six degrees of freedom of the components (Figure 6.16). LTDs were fixed to the ends of the input and output MT connectors, and secured with custom fixturing so that they could be cantilevered out over the sample, where they were lowered into the insertion pockets machined into the optical layer (Figure 6.17 and Figure 6.18). The micrometer actuators allowed for the LTDs to be manually positioned into alignment with the waveguide packets, with optical power transmission serving as the feedback component for proper alignment.

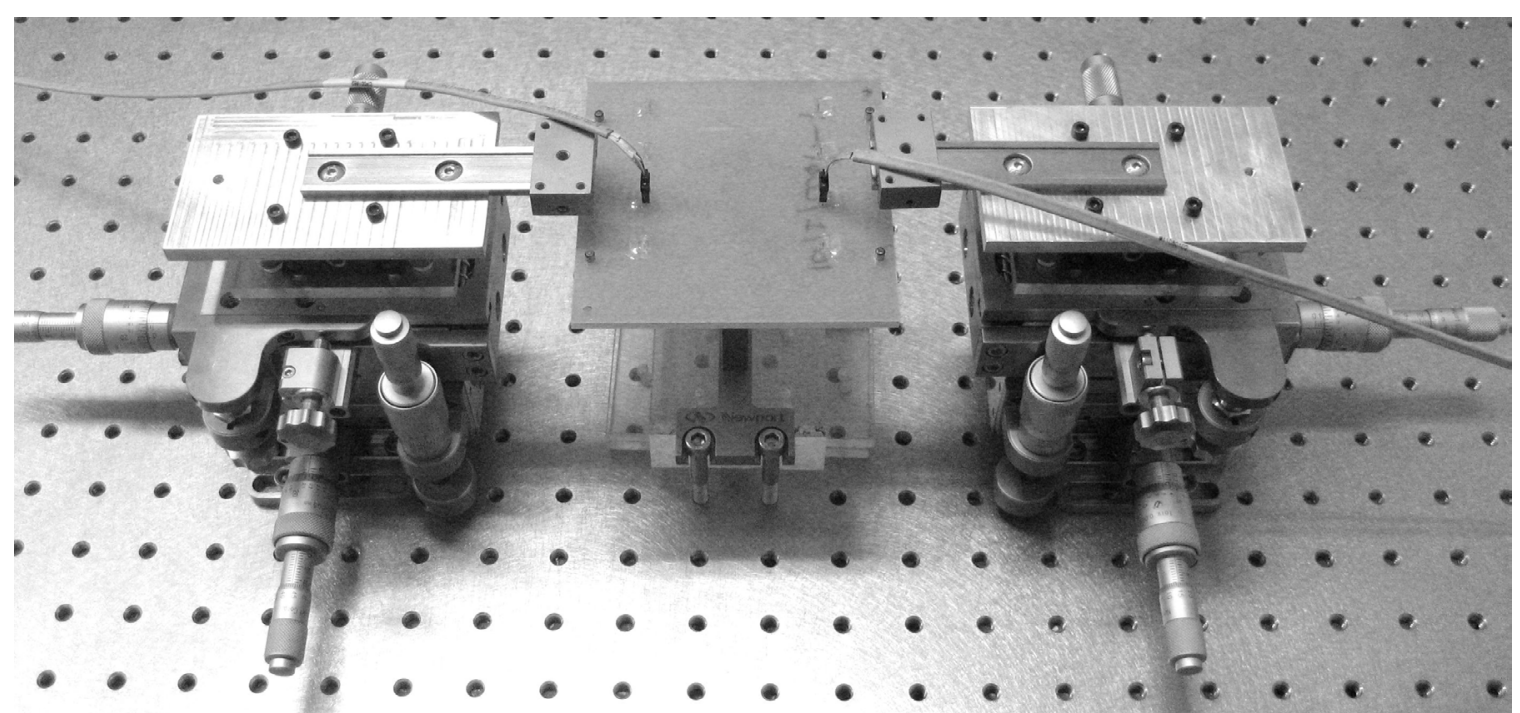

Figure 6.16. Manually operated alignment stages used to precisely position the LTDs during insertion into the optical layer. 


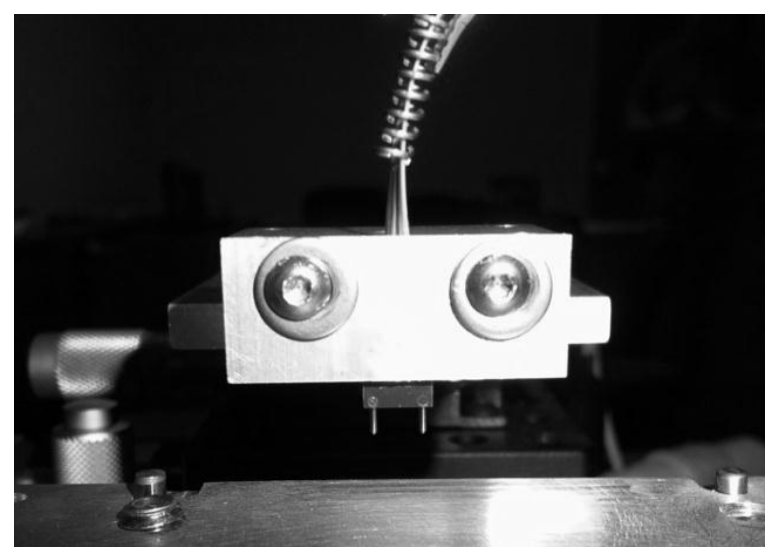

Figure 6.17. Custom mounting fixture used to secure the input and output cabling for positioning with respect to the optical layer.

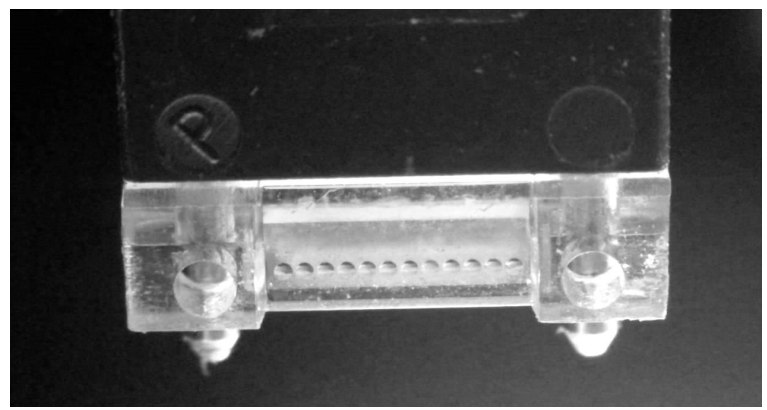

Figure 6.18. LTD is secured to the clamped MT connector for precision optical alignment with respect to the optical layer.

Proper alignment and performance balance among the 12 optical channels were achieved through a repeated process of position correction using optical loss as feedback, diagrammed in Figure 6.19. Adjustment in the vertical and lateral position, with respect to the waveguide path, had the greatest impact on overall performance, and the rotation of the LTD about the waveguide path axis had the greatest impact on performance balance between the waveguide channels (Figure 6.20). The separation distance between the waveguide end-faces and the LTD, and the remaining two degrees of rotation had noticeably higher tolerance and thus made less of an impact on overall performance. These parameters were adjusted as a way to "fine-tune" the system. 


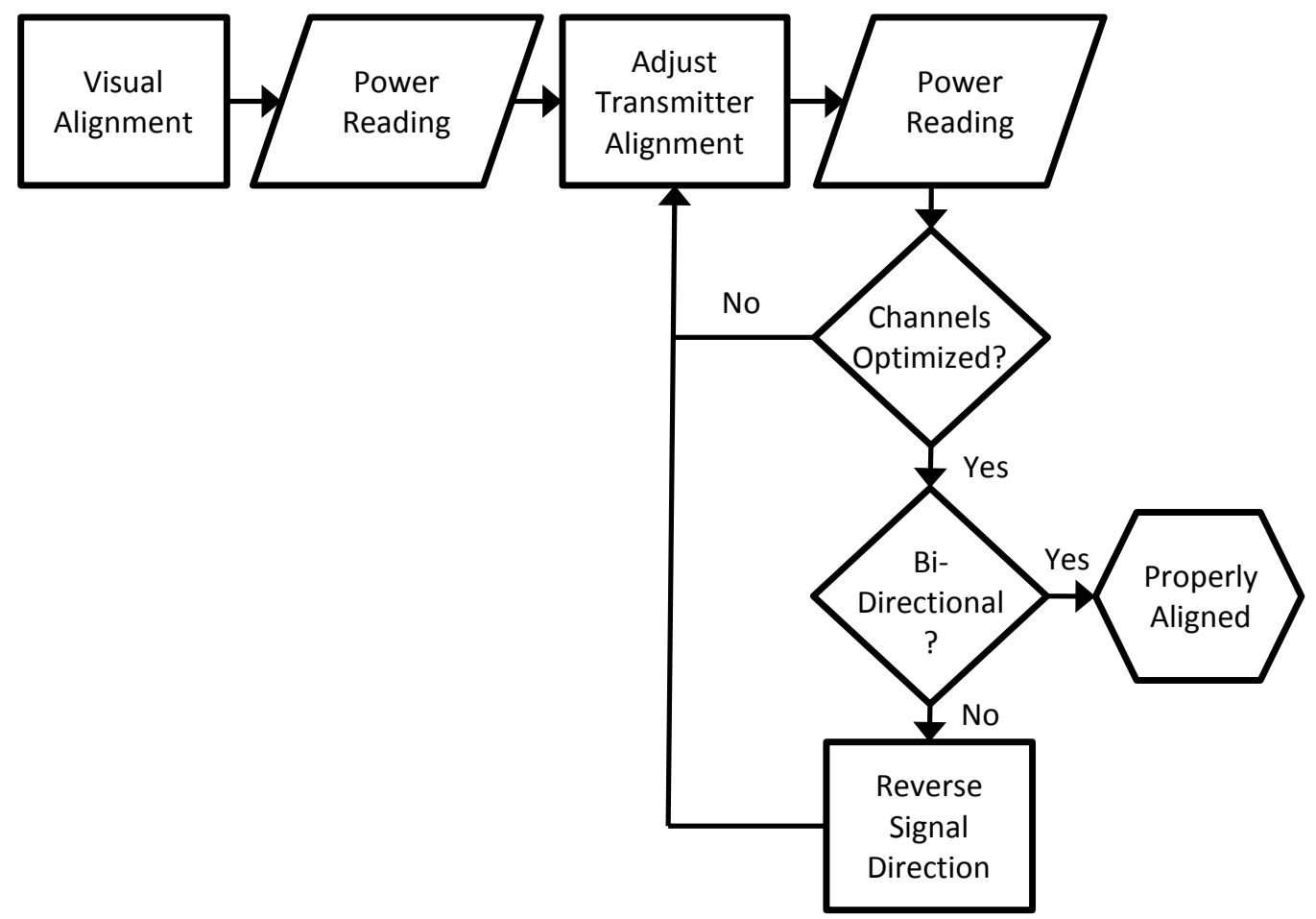

Figure 6.19. LTD insertion procedure control loop ensured proper alignment between the LTDs and the embedded optical waveguides.
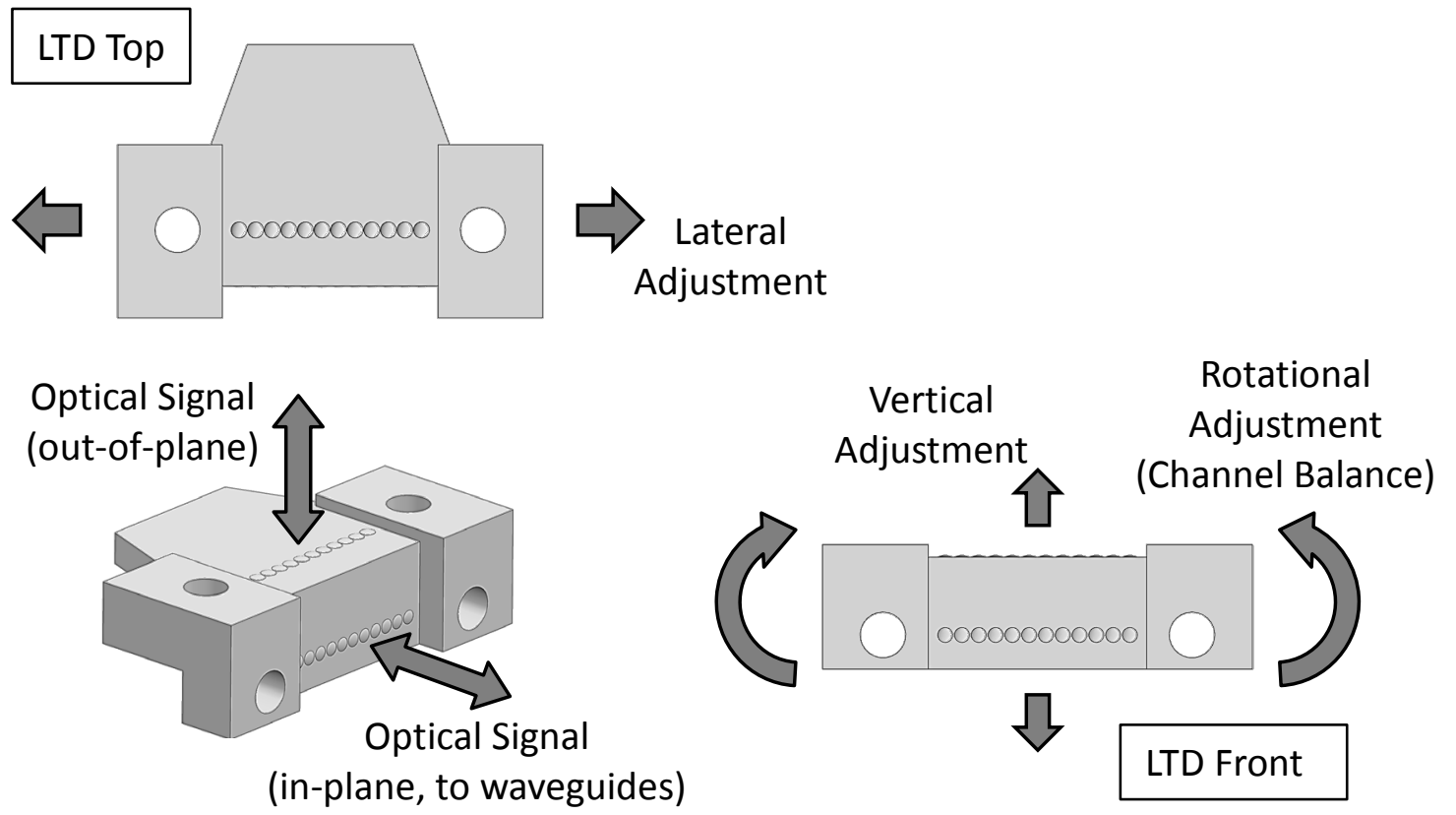

Figure 6.20. All six degrees of freedom for the LTD were adjustable, but the three indicated parameters played the most significant role in performance. 
After the LTDs were properly positioned, they were epoxied into place through a twostep epoxy process. The first step was to apply small amounts of a UV curable optical adhesive (Norland Optical Adhesive 68T) at three discrete locations to "tack" the component into place (Figure 6.21). NOA 68T was chosen because of its high viscosity, which reduced the risk of it flowing into unwanted areas of the LTD. It was also able to be cured in place quickly using a high irradiance UV source, reducing the chances of the LTD shifting during the curing process. NOA 68T cannot withstand the temperature extremes that are required for the OEPWB, but it was effective in quickly securing the LTDs in place allowing for the application of a second, more robust two-part epoxy (B481 TH by Reltek) to the assembly. This "tacking" process, completed in minutes, helped reduce any movement of the LTDs during the 12 hour curing process of the B- $481 \mathrm{TH}$, which was critical to maintaining optical alignment with the waveguides. B-481TH is a thixotropic blend that was chosen because it is thermally stable over a wide temperature range $\left(-40^{\circ} \mathrm{C}\right.$ to $\left.110^{\circ} \mathrm{C}\right)$ and its high viscosity makes it less likely to flow undesirably into the areas of the LTD which contain free space optics. B-481TH performed very well in initial bonding studies, securely adhering to polycarbonate (receivers), FR4, and siloxane (which is difficult to bond to) making it very suitable for this application. Epoxy was applied to the sides and back gate portion of the LTD (Figure 6.21), carefully and sparingly to not risk any excess epoxy flowing onto the lenses or the face of the total internal reflection mirror. The epoxy was allowed to cure at room temperature overnight, as adding any heat would promote unwanted material flow.
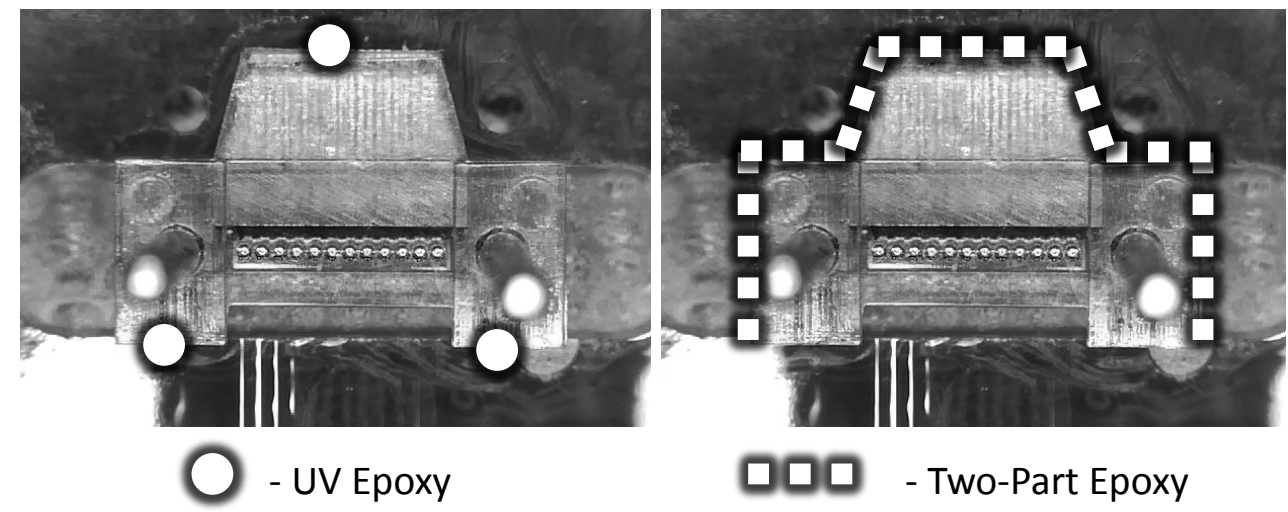

Figure 6.21. Locations of UV and final, two-part epoxy deposits.

The last step, after the LTDs were secured within the optical layer, was to epoxy the receivers over the LTD sites using the same two-step epoxy process. The receivers were "tacked" at the corners using NOA 68T, and then sealed at the base around the entire perimeter using B-481TH. The complete component stack can be seen schematically in Figure 6.22. After B-481TH was applied to the base of all of the receivers, the samples were again allowed to cure at room temperature for 10 hours, after which, they were baked at $60^{\circ} \mathrm{C}$ for 10 hours to ensure complete curing throughout the bulk of the epoxy. 


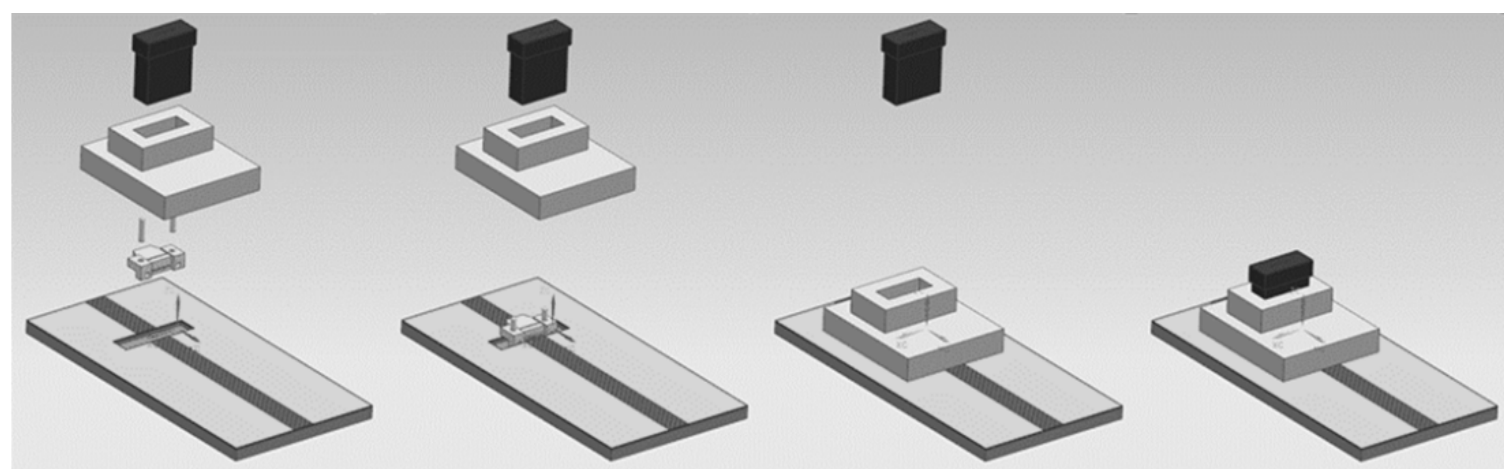

Figure 6.22. Optical component stack comprised of optical layer, LTD, connector receiver, and MT connector.

\subsection{Results}

\subsubsection{Initial Characterization}

Three OEPWB prototypes were fabricated, marked A, B, and C, in order of completion. Sample $\mathrm{C}$ exhibited the highest quality and consistency of the three, according to initial output measurements. The performance of Samples A and B was lower due to complications in the two-part epoxying step, especially so for Sample A (the first of the batch). When applying the two-part epoxy coating to the LTDs, unwanted epoxy flow led to the LTD mirrors becoming coated. Attempts were made to remove this unwanted epoxy using IPA, but a film was left behind that coated the LTD optics, causing the performance to suffer. The process improved with each iteration, as it was observed that layering up small amounts of the two-part epoxy was more effective at reducing epoxy flow than applying one substantial coating.

Upon completion, each waveguide was tested independently by Calumet Electronics Corporation (CEC) and MTU for optical loss to evaluate device functionality and to obtain baseline performance measurements. Results were agreeable between CEC and MTU, and they showed that performance improved with each of the three samples as the fabrication process was refined, detailed in Table 6.2. The average total system losses were calculated using the average output power for each sample, according to:

$$
\text { Optical Loss }(d B)=-10 * \log \left(\frac{\text { Average Output Power }}{\text { Input Power }}\right)
$$

Sample A had a total loss average of $9.36 \mathrm{~dB}$, the highest of the three samples. Sample B had a total loss average of $8.50 \mathrm{~dB}$, reflecting the improvements in the fabrication process, and the best results were seen in Sample C, with a total loss average of $6.59 \mathrm{~dB}$, which is well below the $10 \mathrm{~dB}$ design threshold. 
Table 6.2

Initial output power (and calculated optical loss) results for Samples A, B, and C, obtained from MTU and CEC. Loss measurements calculated using $2 \mathrm{~mW}$ input power.

\begin{tabular}{|c|c|c|c|c|c|c|}
\hline Performance & \multicolumn{2}{|c|}{ Sample A } & \multicolumn{2}{c|}{ Sample B } & \multicolumn{2}{c|}{ Sample C } \\
\hline (Based on & $\begin{array}{c}\text { Output } \\
\text { mW input) } \\
(\mu \mathrm{W})\end{array}$ & $\begin{array}{c}\text { Optical } \\
\text { Loss } \\
(\mathrm{dB})\end{array}$ & $\begin{array}{c}\text { Output } \\
\text { Power } \\
(\mu \mathrm{W})\end{array}$ & $\begin{array}{c}\text { Optical } \\
\text { Loss } \\
(\mathrm{dB})\end{array}$ & $\begin{array}{c}\text { Output } \\
\text { Power } \\
(\mu \mathrm{W})\end{array}$ & $\begin{array}{c}\text { Optical } \\
\text { Loss } \\
(\mathrm{dB})\end{array}$ \\
\hline Set 1 Average & 213.3 & 9.7 & 156.0 & 11.1 & 392.1 & 7.1 \\
Set 2 Average & 172.2 & 10.7 & 304.2 & 8.2 & 342.4 & 7.7 \\
Set 3 Average & 114.2 & 12.4 & 286.0 & 8.4 & 552.8 & 5.6 \\
Set 4 Average & 427.2 & 6.7 & 384.6 & 7.2 & 466.2 & 6.3 \\
Total Average & 231.7 & 9.4 & 282.7 & 8.5 & 438.4 & 6.6 \\
Std. Dev. & 155.6 & & 145.6 & & 196.6 & \\
\hline
\end{tabular}

After baseline measurements were obtained, the samples were submitted to Lockheed Martin for environmental testing. Lockheed Martin repeated baseline testing upon receiving the devices and reported higher loss measurements $(0.4$ to $4.2 \mathrm{~dB})$ for each of the waveguide sets (Table 6.3), though the reason for this was not determined. One possible contributor is the difference in test setups, as MTU and CEC utilize similar equipment, while the setup at Lockheed Martin incorporates optical switches for indexing between the 12 channels in each group.

Table 6.3

Comparison between initial testing conducted by MTU/CEC and Lockheed Martin (LM).

\begin{tabular}{|c|c|c|c|c|c|c|}
\hline Performance & \multicolumn{2}{|c|}{ Sample A } & \multicolumn{2}{c|}{ Sample B } & \multicolumn{2}{c|}{ Sample C } \\
\hline $\begin{array}{c}\text { (Based on } \\
2000 \mathrm{~mW} \\
\text { input) }\end{array}$ & $\begin{array}{c}\text { MTU/ } \\
\text { CEC } \\
\text { Results } \\
(\mathrm{dB})\end{array}$ & $\begin{array}{c}\text { LM } \\
\text { Results } \\
(\mathrm{dB})\end{array}$ & $\begin{array}{c}\text { MTU/ } \\
\text { CEC } \\
\text { Results } \\
(\mathrm{dB})\end{array}$ & $\begin{array}{c}\text { LM } \\
\text { Results } \\
(\mathrm{dB})\end{array}$ & $\begin{array}{c}\text { MTU/ } \\
\text { CEC } \\
\text { Results } \\
(\mathrm{dB})\end{array}$ & $\begin{array}{c}\text { LM } \\
\text { Results } \\
(\mathrm{dB})\end{array}$ \\
\hline Set 1 Average & 9.7 & 13.1 & 11.1 & 15.3 & 7.1 & 10.5 \\
Set 2 Average & 10.7 & 11.0 & 8.2 & 11.9 & 7.7 & 11.2 \\
Set 3 Average & 12.4 & 15.0 & 8.4 & 11.5 & 5.6 & 8.7 \\
Set 4 Average & 6.7 & 10.2 & 7.2 & 9.4 & 6.3 & 9.9 \\
\hline
\end{tabular}

Also included in the baseline testing at Lockheed Martin was the cross talk between adjacent waveguides. Each interconnection has neighboring connections that may interfere with optical signal transmission, which is called optical cross talk. To measure optical cross talk, a signal was transmitted through each of the twelve input signals provided by the MT, and a measurement was taken of the adjacent paths. These paths were termed as, for example, SplB3-minus or SplB3-plus. This example description 
name represents the third MT location of Sample B and the measurement of the one less and one greater side in the MT ferrule position. Figure 6.23 shows that all 528 measurements of cross talk had greater than $30.5 \mathrm{~dB}$ isolation, which would not translate to signal interference due to sensitivity. The data was verified for a variety of configurations (signal direction, MT orientation) to determine a difference between board to board, MT to MT, and between ferrule positions. ANOVA statistical analysis did reveal that there was no statistical difference in signal direction on cross talk isolation, seen in Figure 6.24.

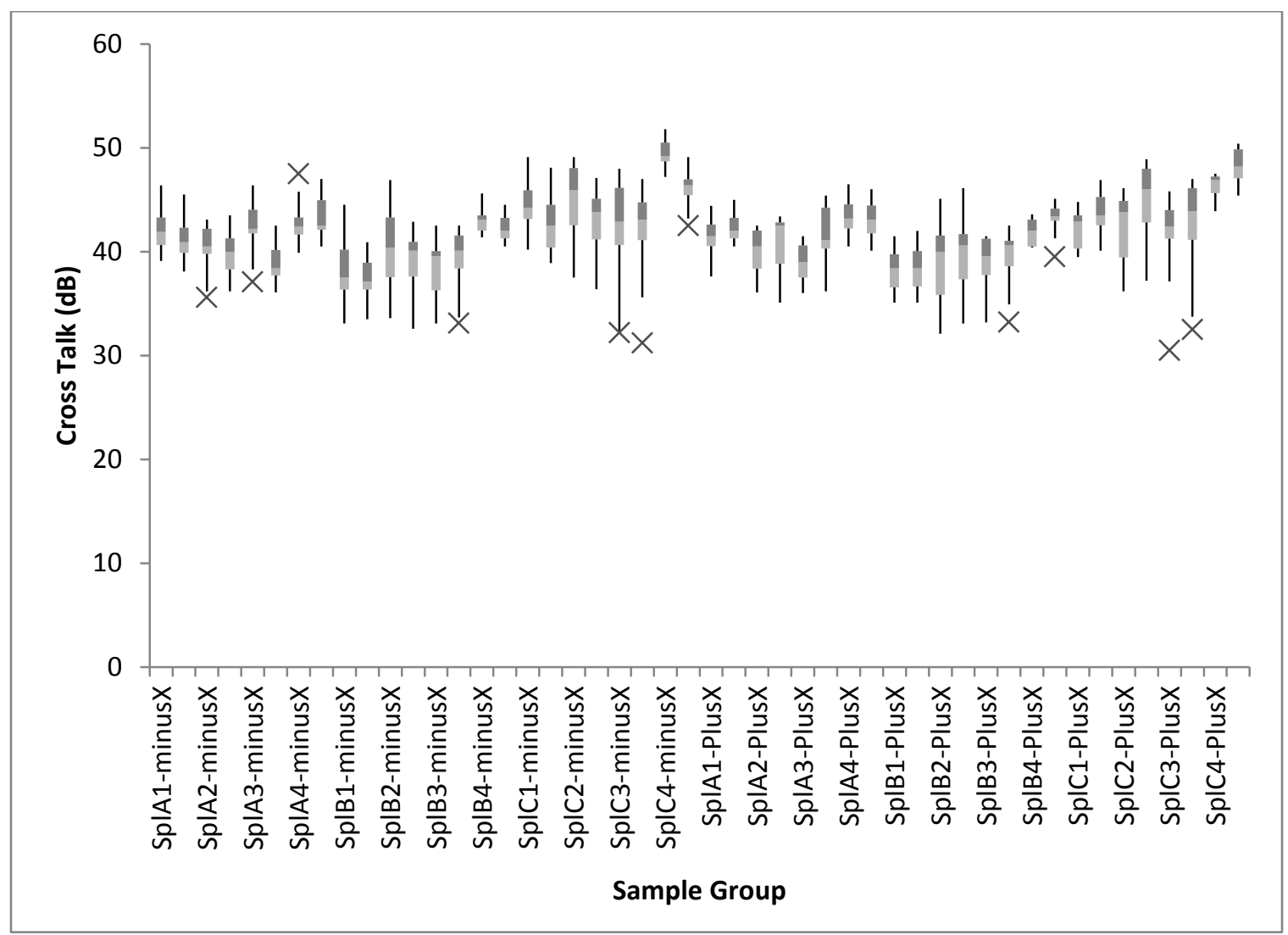

Figure 6.23. Cross talk measurements for each of the four waveguide groups on the three individual samples.

A closer look at the data revealed one significant difference between sets. There are two types of circuits on the optical layer pattern; those that have waveguides separated on 250 $\mu \mathrm{m}$ pitch and those that come closer together in the routing area to a pitch of $100 \mu \mathrm{m}$. The slight difference between the signals for these two pitch values was validated by ANOVA statistical analysis, illustrated in Figure 6.24, with nearly a $5 \mathrm{~dB}$ greater isolation for waveguides with a pitch of $250 \mu \mathrm{m}$ over those with a pitch of $100 \mu \mathrm{m}$. 


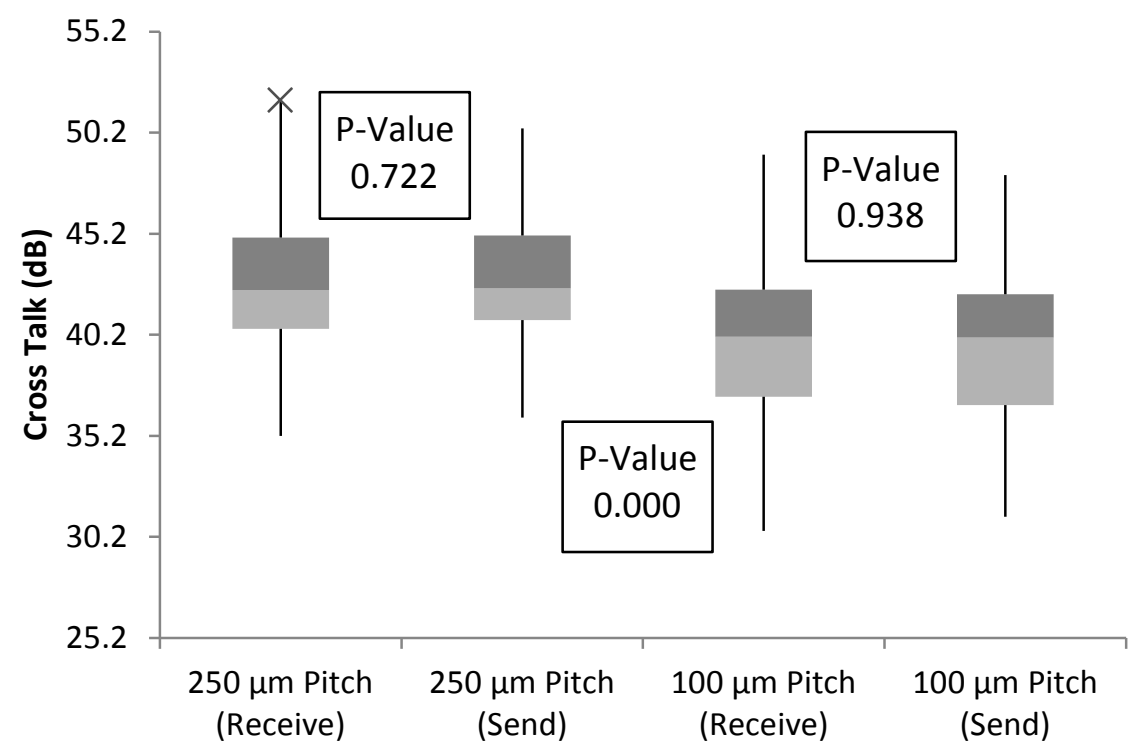

Figure 6.24. Cross talk difference between circuit isolation differences $250 \mu \mathrm{m}$ versus $100 \mu \mathrm{m}$. ANOVA statistical analysis shows that there is no significant difference in signal direction (between "send" and "receive"), as $\mathrm{P}>0.05$. There is, however, a significant difference in cross talk when comparing pitch values, as $\mathrm{P}<0.05$.

\subsubsection{Environmental Testing}

The intent of the environmental testing was to validate the prototype technology and ensure functionality in the harsh conditions seen in an avionics setting. The first round of environmental exposure subjected the OEPWBs to thermal cycling over increasingly stringent ranges, starting at $0^{\circ} \mathrm{C}$ to $70^{\circ} \mathrm{C}$, followed by $-20^{\circ} \mathrm{C}$ to $85^{\circ} \mathrm{C}$, and finally the most extreme range of $-40^{\circ} \mathrm{C}$ to $85^{\circ} \mathrm{C}$ (refer to MIL-STD-883 Method 1010, United States Military Standard as a reference). At each temperature range, the samples were subjected to 100 , one hour cycles. Optical loss was measured after ten cycles and 100 cycles, and once in between, when possible (after 80 cycles for $-20^{\circ} \mathrm{C}$ to $85^{\circ} \mathrm{C}$ and after 51 cycles for $-40^{\circ} \mathrm{C}$ to $85^{\circ} \mathrm{C}$ ). In an avionics setting, temperature change can be dramatic with the significant variation in altitudes, so it was important to evaluate board operation over a fluctuating range of temperatures.

After thermal cycling, the samples were subjected to temperature-humidity cycling, where the OEPWB had to withstand exposure to water dripping from overhead. If board level waveguide technology is to be viable in avionics, it must be able to survive inclement weather conditions such as precipitation and salt water spray. The temperature humidity test consisted of ten, 24 hour cycles, with optical loss being measured after the fifth and tenth cycles, detailed in Figure 6.25 (refer to MIL-STD 810F, United States Military Standard as a reference). The relative humidity in the testing chamber was $95 \pm 4 \%$, except in the descending phase in which humidity might drop to $85 \%$. 
The final evaluation was environmental aging life testing, where the prototypes were subjected to $110^{\circ} \mathrm{C}$ for 216 hours, after which optical loss was measured (refer to TIA455-4, Telecommunications Industry Association as a reference). Accelerated aging is used to determine the long term effects of extreme conditions in a shortened time frame.

Due to time and budget constraints, shock and vibration testing was unable to be completed, but is a critical component to the evaluation of OEPWBs for avionics. With only three prototypes, it was important to spread the testing out strategically to obtain sufficient performance representation. Table 6.4 shows which of the three submitted prototype samples were used for each of the completed environmental performance tests.

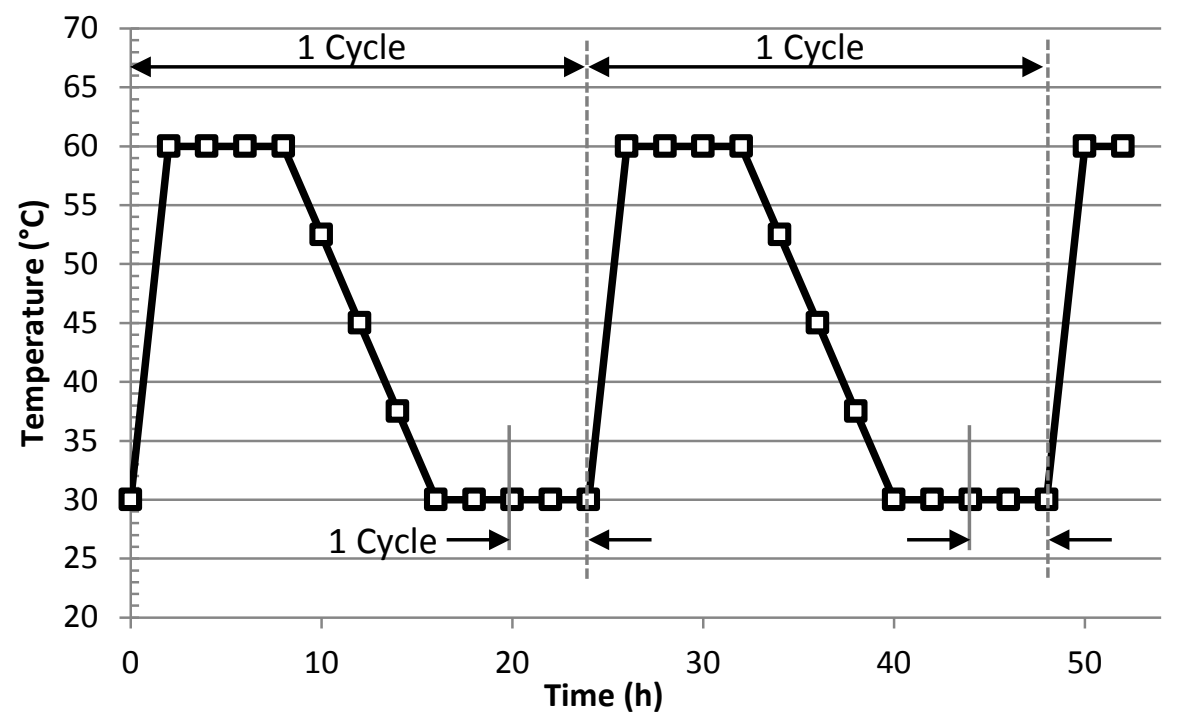

Figure 6.25. Temperature humidity cycling sequence provided by Lockheed Martin. 
Table 6.4.

Samples used for various environmental testing.

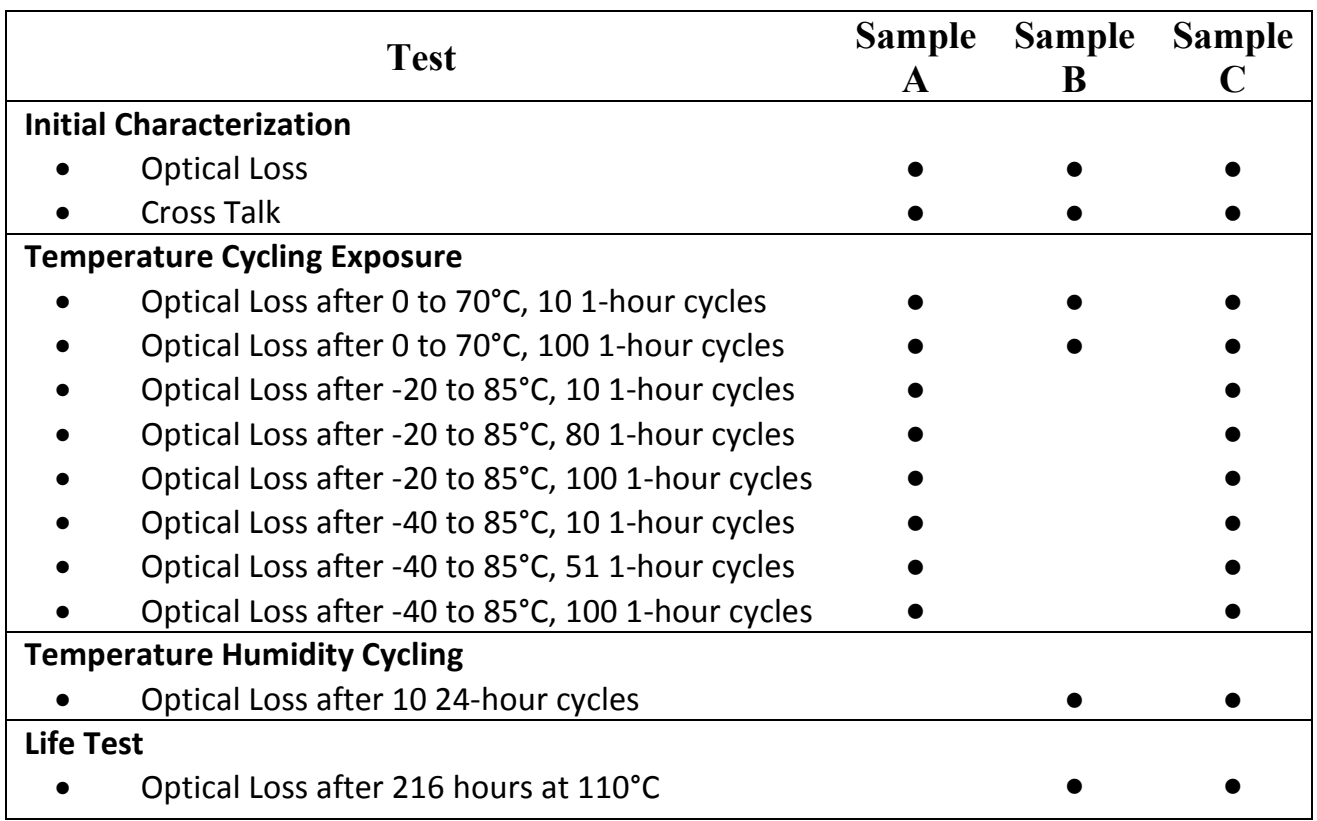

The environmental tests yielded useful information that served as feedback for current fabrication techniques and offered insight for possible screening measures for future pilot production manufacturing. Figure 6.26, Figure 6.27, and Figure 6.28 detail the performance of the OEPWB assemblies through the various environmental excursions. Figure 6.26 illustrates that not all channels on Sample A were able to completely withstand the thermal cycling beyond the first temperature range of 100 cycles at $0^{\circ} \mathrm{C}$ to $70^{\circ} \mathrm{C}$. Lockheed Martin reported that two of the eight receivers (one from each of two different channels) had delaminated from the sample as a result of the thermal cycling, severely degrading the performance of the part. As detailed earlier, this sample experienced complications during the two-part epoxy phase, a factor that could have led to this delamination, and the performance breakdown seen in the latter two temperature regimes. It is positive to note that in spite of these defects, no failures arose over the course of 100 cycles at $0^{\circ} \mathrm{C}$ to $70^{\circ} \mathrm{C}$, a temperature range more commensurate with commercial data communications.

As expected, Samples B and C performed significantly better (Figure 6.27 and Figure 6.28 , respectively), both surviving the first range of thermal cycling as well as the temperature humidity environment. Importantly, this indicates that the receivers were effective in shielding the LTD cavity from outside contamination. Sample C saw little to no performance degradation over the entirety of the thermal cycling, surviving the most extreme range of $-40^{\circ} \mathrm{C}$ to $85^{\circ} \mathrm{C}$. Both Samples B and $\mathrm{C}$ saw significant degradation in performance with the life testing after 216 hours of exposure, but suffered no component delaminations. 


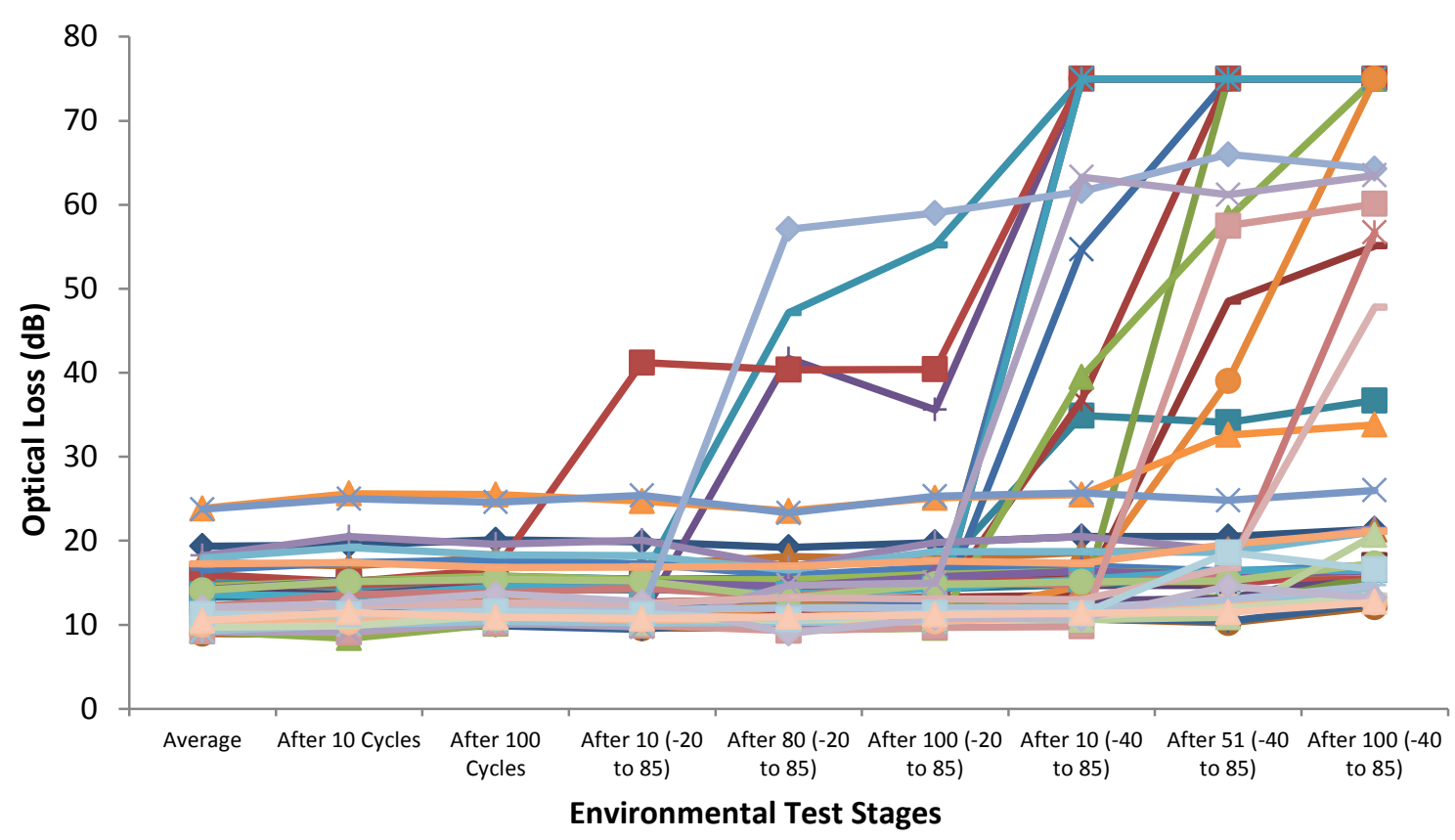

Figure 6.26. Optical loss results for Sample A after each round of environmental testing, with all 48 connectorized waveguides represented. Failures began to increase as stages of thermal cycling became more stringent.

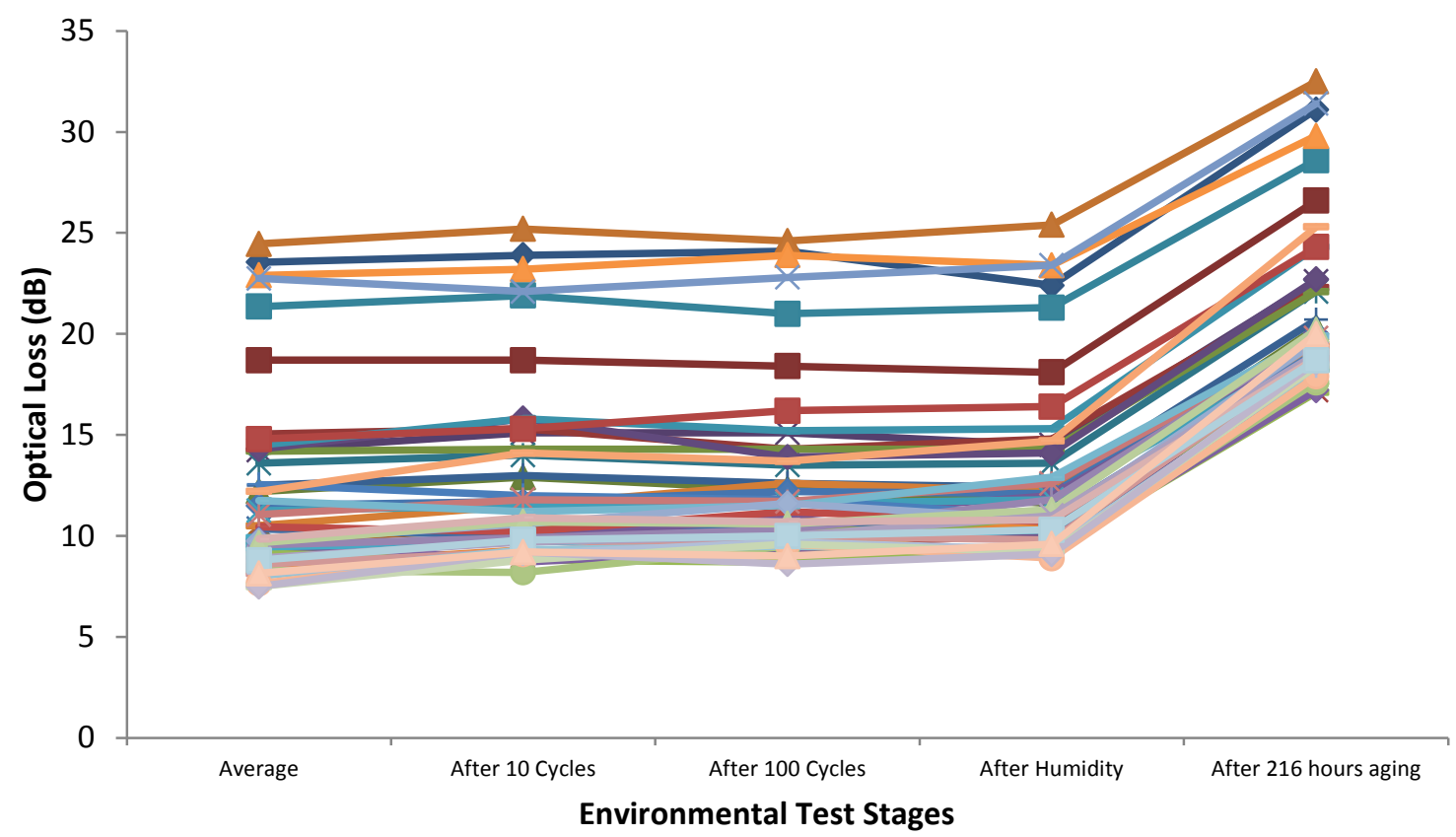

Figure 6.27. Optical loss results for Sample B after each round of environmental testing, with all 48 connectorized waveguides represented. Sample survived 100 cycles of $0^{\circ} \mathrm{C}$ to $70^{\circ} \mathrm{C}$ and temperature humidity cycling. Performance significantly decreased with accelerated aging at $110^{\circ} \mathrm{C}$. 


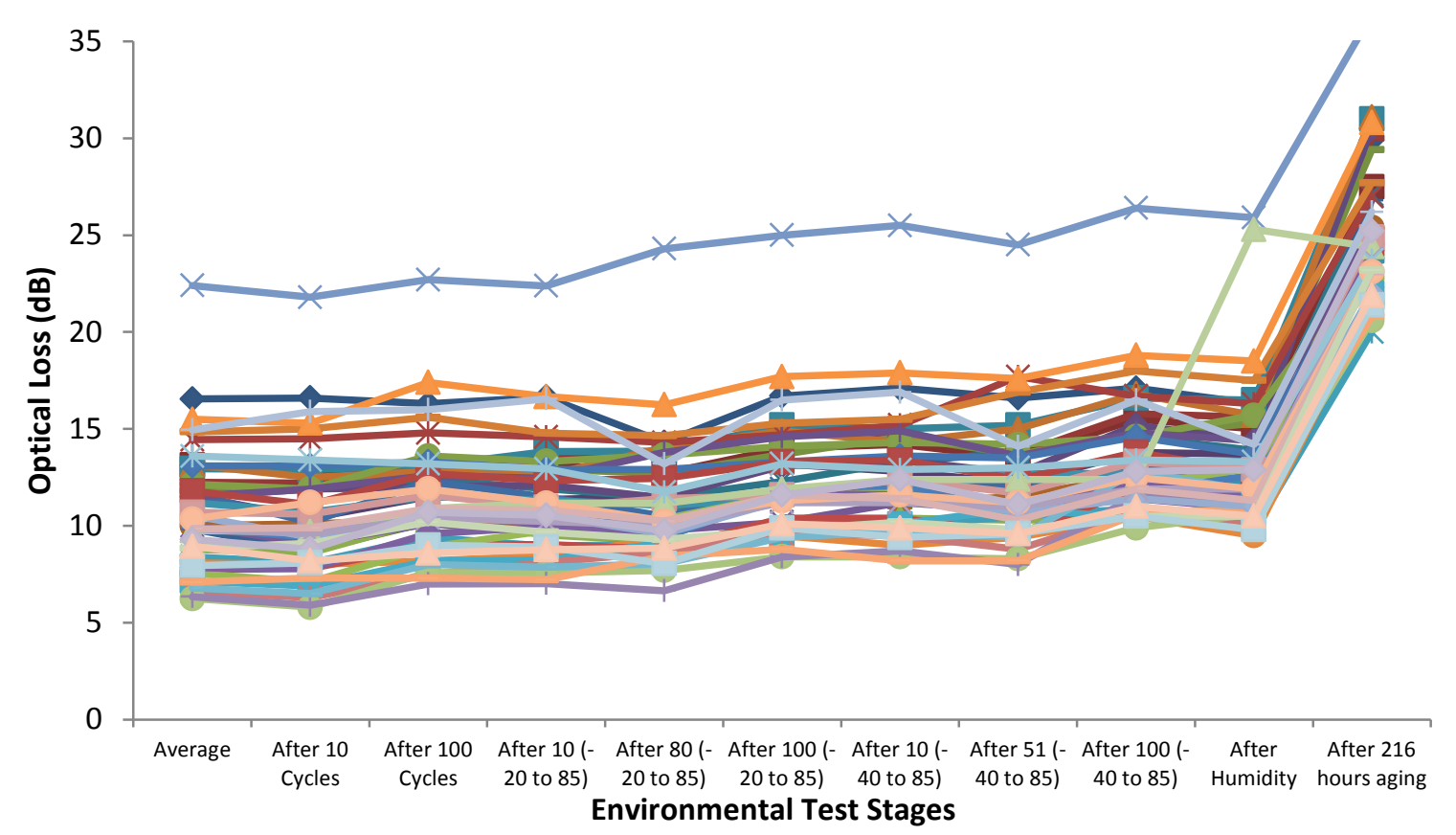

Figure 6.28. Optical loss results for Sample $\mathrm{C}$ after each round of environmental testing, with all 48 connectorized waveguides represented. Sample survived 100 cycles of $-40^{\circ} \mathrm{C}$ to $85^{\circ} \mathrm{C}$ and temperature humidity cycling. Performance significantly decreased with accelerated aging at $110^{\circ} \mathrm{C}$.

\subsection{OEPWB Conclusions and Future Work}

An optical layer was patterned according to customer designs provided by Lockheed Martin, and light turning devices were successfully implanted at appropriate locations through developed alignment and bonding procedures. Several connector receiver types were fabricated and evaluated to different levels, including receivers capable of securing standard MT connectors, spring loaded MTP connectors, and USCONEC PRIZM right angle connectors. Three prototype OEPWBs were successfully fabricated, achieving total system optical losses below the $10 \mathrm{~dB}$ design limit according to internal testing. These prototypes were evaluated over severe environmental conditions, surviving 100, 1 hour cycles at $-40^{\circ} \mathrm{C}$ to $85^{\circ} \mathrm{C}$, as well as thermal cycling in a $95 \%$ humidity environment.

Embedding the optical layer itself also offers the opportunity for improved device reliability as it has been demonstrated that embedded optical layers are less susceptible to rapid changes in temperature when compared to surface layers (Investigation of Environmental Reliability of Optical Polymer Waveguides Embedded on Printed Circuit Boards, 2007). A thin FR4 or resin layer would help protect the waveguides from mechanical damage and would result in more balanced stresses at the top and bottom of the optical layer during thermal cycling. 
Complications encountered in bonding the LTDs and receivers in place have highlighted room for improvement in the design of the component stack. The current LTD makes use of free space optics which require open air space for proper operation. The fabrication process, and overall reliability of the device, would be improved if an alternative could be developed. This could be accomplished through the use of gradient index (GRIN) lenses, which have flat faces and do not require open air space for proper operation. This would allow them to be butt coupled and bonded to the waveguide end faces, eliminating the risk of contamination during the epoxy process. Along these lines, if the total internal reflection mirror were metal coated, making it a plain reflector, it would not be affected by epoxy contamination in the fabrication process. With these changes, the LTD insertion pocket could be completely filled with epoxy, protecting the LTD from the ambient environment, improving long term reliability. Another opportunity for improvement in the component stack would be the unification of the LTD and receiver, eliminating any air gaps and the need to physically align these two parts. The unified piece could be injection molded with alignment features specific to the cable connector of choice, making installation and connection a simplified process.

A logical process improvement is the automation of the time consuming "back-and-forth" method of LTD alignment. Using motorized actuators, the repeated physical alignment iterations could be computer automated, using output power (or optical loss) as a feedback parameter. This computer controlled process would result in faster optimization cycles with the ability to log quantifiable data for reference. This could be a largely unmanned process step, drastically increasing throughput.

\subsubsection{Acceptance Test Plan for Manufacturing}

For any new technology developed, an acceptance plan must also be developed to identify early "infant mortality" failures to ensure reliable products are being delivered to the end user. Figure 6.29 illustrates the classic reliability curve, where the observed failure rate decreases in the early stages as the "infant mortality" rate falls, followed by a period of fairly constant rate of failure, and finally graduating to a period of increasing failure as parts near the end of their life cycle and begin to wear out. It is important that the parts are in the middle region of constant failure before they are delivered to the end user so that they are dependable. To accomplish this, it is critical to identify certain physical or performance tests which identify these early failures as efficiently as possible. 


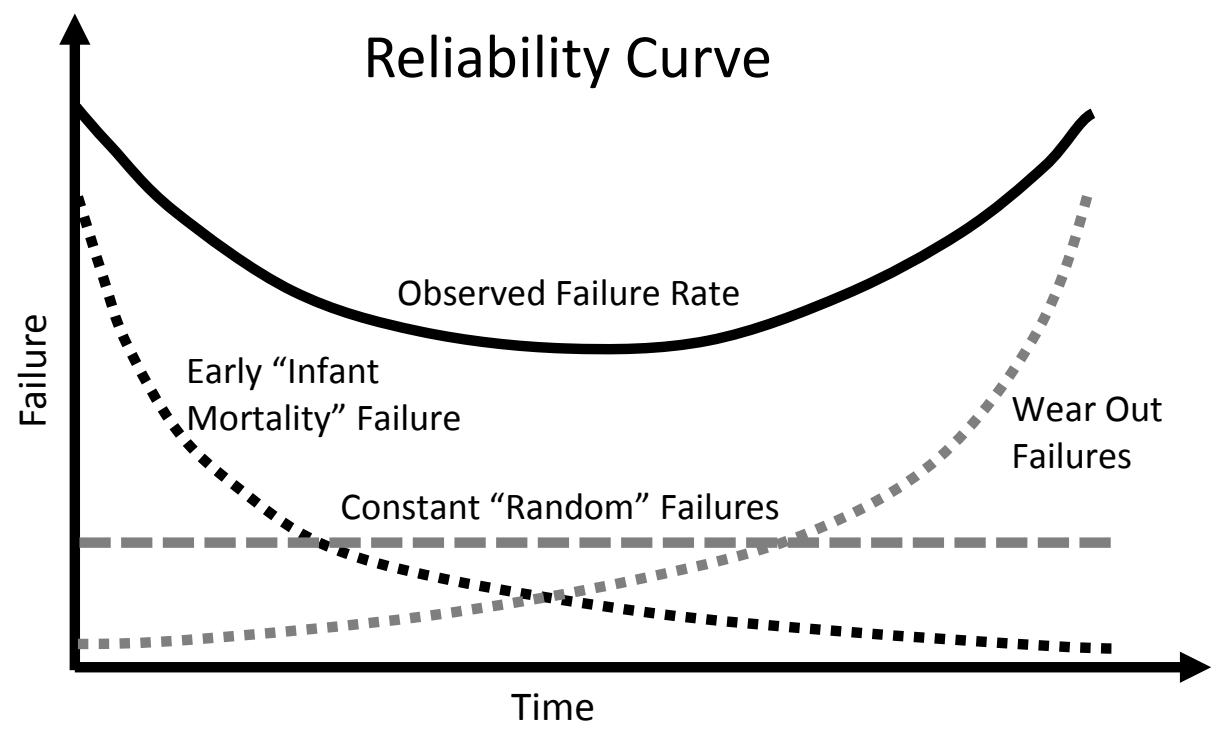

Figure 6.29. Reliability curve charting part failures.

With the separation of the electrical and optical layers, proven practices for detecting failures in manufactured printed circuit boards can remain in place, a significant benefit to this approach to building OEPWBs. If an electrical layer fails, it can be discarded before it is paired with the optical layer, or vice versa. This cuts down on the waste that would be seen if these layers were not created and tested separately.

Testing and characterization work has shown relative stability in the polymer waveguide structures and accompanying interconnect components of the optical board throughout humidity testing and thermal cycling over the range of $0^{\circ} \mathrm{C}$ to $70^{\circ} \mathrm{C}$, so they would be poor candidates for early failure detection. Life span aging is too time intensive and brought about failure across the board, making it a poor choice as well. Failures did begin to arise and increase in Sample A as thermal cycling entered the more stringent stages, especially in the $-40^{\circ} \mathrm{C}$ to $85^{\circ} \mathrm{C}$ period of testing (Figure 6.26), as it was noted that the epoxy bonds began to fail. Dow Corning advised that many two-part epoxies (used to secure the LTD and connector receptacle within the board) do not handle extreme negative temperatures well. The optical failures seen in Sample A point to defective bonding which was not present in Sample $\mathrm{C}$, as it was able to withstand the most extreme temperature range of $-40^{\circ} \mathrm{C}$ to $85^{\circ} \mathrm{C}$. This would signify that there is a high interaction with stringent thermal cycling and early failures, making it an excellent candidate for early defect detection and should thus be the basis of the environmental test screening portion of the acceptance test plan. This can be an unmanned process step that can run continuously to its completion. Optical loss would be measured before and after cycling, and the results compared to detect channel failures.

Based on this interaction, future work should focus on performing an analysis with a more significant sample base, to determine the Environmental Stress Screening, or ESS, 
temperature range and number of cycles (limited, eg. 10) required to identify early failures. These determinations would be made with the use of a Weibull probability plot, useful for process development testing where large sample sizes are not yet available. A typical Weibull plot is illustrated in Figure 6.30, where the horizontal scale is a measure of life or aging (start/stop cycles, operating time, or mileage) and the vertical scale is the cumulative percentage failed (Abernethy, 1993).

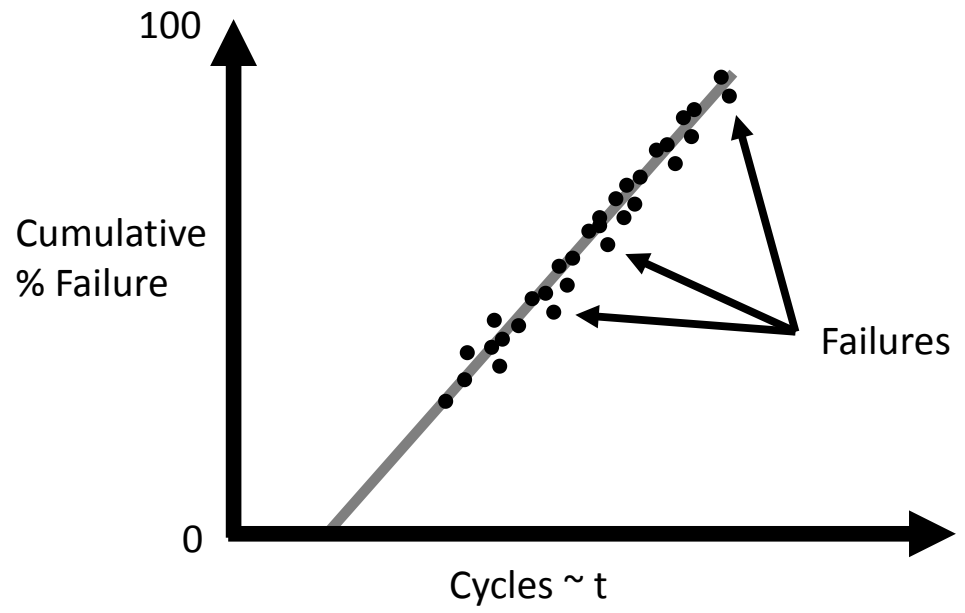

Figure 6.30. A typical Weibull plot, where the horizontal scale is a measure of life or aging (start/stop cycles, operating time, or mileage) and the vertical scale is the cumulative percentage failed (Abernethy, 1993). Weibull Plot would be used in determining acceptable test parameters for early failure detection. 


\section{Chapter 7. Conclusion and Recommendation}

The main goals of this research were the development and characterization of direct write material deposition and patterning tools for the fabrication of optical waveguide systems on large substrates, and the development of out-of-plane coupler components with integrated connectors to secure external fiber optic cabling for the fabrication of fully functional OEPWBs. A direct dispense tool was designed, assembled, and characterized for the repeatable dispensing of blanket waveguide layers over a range of thicknesses $(25-225 \mu \mathrm{m})$, with the ability to achieve thicknesses above and below this range, eliminating waste material and affording the ability to utilize large substrates. This tool was used to directly dispense multimode waveguide cores which required no UV definition or development. These cores had circular cross sections with initial attenuation loss ranging from $0.06 \mathrm{~dB} / \mathrm{cm}$ to $0.09 \mathrm{~dB} / \mathrm{cm}$, which is comparable to lithographically fabricated square waveguides. A laser direct write tool was designed, assembled, and characterized for direct write patterning waveguides that were comparable in quality to those produced using standard lithographic practices $(0.047 \mathrm{~dB} / \mathrm{cm}$ for laser written waveguides compared to $0.043 \mathrm{~dB} / \mathrm{cm}$ in the lithographically patterned waveguides). Commercially available LTDs were implanted into the optical layer for out-of-plane routing of the optical signals and protective connectors were developed to shield the LTDs from environmental contamination and to secure external fiber optic cabling. Alternative connectors were developed to improve upon the form factor of the interface. Fully functional OEPWBs were fabricated featuring input and output out-of-plane optical signal routing capable of receiving standard MT style connectors with total optical losses not exceeding $10 \mathrm{~dB}$. These prototypes survived thermal cycling $\left(100\right.$ cycles at $-40^{\circ} \mathrm{C}$ to $85^{\circ} \mathrm{C}$ ) and humidity exposure (ten, 24 hour cycles at $95 \pm 4 \%$ humidity), showing minimal degradation in optical performance. Operational failure occurred after environmental aging life testing at $110^{\circ} \mathrm{C}$ for 216 hours.

Advancements in component design and processing procedures offer great opportunities for performance improvement. The current interconnect relies on several separate components that are each individually aligned and epoxied together. Combining the LTD and the connector into a single molded part decreases the opportunities for misalignment or epoxy contamination. Redesign of the LTD would also allow for the inclusion of specific, segregated "epoxy zones", protecting any free space optics from flowing epoxy. Offering greater benefit would be a shift from the free space optics currently employed in the LTD design. Free space optics are too susceptible to contamination and too difficult to clean once affected, making them ill-suited for use in harsh environments.

Incorporation of gradient index lenses as an alternative to the current spherical lenses would mitigate the challenges of using free space optics, allowing for butt coupling of the LTD to the waveguide end-faces, as well as to the MT or PRIZM connector. Uncured core material could then be used as a bonding medium between the LTD and the waveguides. The core material is well suited for planarization of the waveguide end- 
faces, and its use as an interface would eliminate the need for any polishing steps while also improving optical performance by providing direct coupling. The inclusion of GRIN lenses, along with metallization of the reflector face prior to implantation would enable the insertion pocket to be completely filled with epoxy material without inhibiting optical performance, as the reflector would no longer operate by total internal reflection. Encasing the LTD within the pocket would decrease the risk of debonding due to environmental stresses. These component improvements would significantly decrease the failure modes associated with embedding LTDs into the optical layer, specifically easing the epoxy step. The design shift away from free space optics would result in a more robust interconnect that is less susceptible to shift or contamination.

The LTD alignment process is labor intensive and requires much training and experience to be successful. This process could easily be automated using motorized actuators with output power serving as the feedback mechanism. Optical switches would allow for waveguide channel indexing as part of the system automation, resulting in unmanned LTD alignment. Automation of this process step would greatly increase throughput and offer detailed performance documentation through data logging.

With the time intensity of the LTD insertion process, detecting waveguide failures early, rather than populating defective circuits, is critically important. Developing an inprocess waveguide characterization procedure would improve reliability by eliminating defective boards prior to population. Top view visual inspection of the waveguides under microscope would identify any waveguide defects due to incomplete development or surface contamination during processing. With the described automated optical performance testing setup, all waveguides could be tested at the board edges prior to machining of the insertion pockets. These measurements would detect waveguide failures and also serve as baseline values during LTD insertion. If it is not possible to edge test the waveguide channels based on the desired pattern, the development and inclusion of a representative test set could suffice for quality assurance purposes.

Another process improvement that would aid in achieving alignment between process steps would be the addition of registration marks within the optical layer. Registration marks could be laser written in the waveguide material at predetermined locations for visual alignment to the waveguides during later steps. Laser written features could also potentially offer physical alignment capability if partnered with future LTD design elements, further refining the LTD insertion process.

The assembled direct write tool is effective, but has its drawbacks. The current setup relies on initial physical contact to reference the tooling to the substrate surface. This process is not ideal, as variances in the substrate thickness or tilt in the workplate holding the substrate can result in inaccurate standoff distances. The inclusion of an integrated surface metrology tool would allow for in-process adjustment of the Z-axis position, resulting in accurate standoff distances for direct dispensing and laser direct writing. The inclusion of this tool could also yield in-process feedback about layer thickness, 
improving the process of direct dispensing blanket layers. Refinement of the direct write process would also be beneficial. Characterization work focused on observing the bounds and limitations of the system, often writing at speeds much higher than necessary. Writing at slow speeds results in more smoother, more controlled motion, which would likely result in improved waveguide performance. Slower write speeds during the direct dispense process would likely lead to more consistent bead shapes, improving the reliability of directly dispensing waveguides.

While it is necessary to address the improvements that can be made on the current fabrication tooling and procedure, it is important to look ahead for opportunities for improvement as the technology progresses to large scale manufacturing. One change in the waveguide fabrication process would be a switch from syringe dispensing to inkjetting or spray coating for material deposition. Material transfer efficiency would decrease slightly, but ink-jetting and spray coating of blanket layers are not susceptible to small variances in standoff distance making them more robust and reliable application methods than syringe dispensing, which has more variables and opportunities for inconsistency. While these methods would not be suitable for the direct dispensing of waveguides, they would offer comparable material savings with greater process automation. 


\section{WORKS CITED}

1. Benner AF, Ignatowski M, Kash JA, Kuchta DM, Ritter MB. Exploitation of optical interconnects in future server architectures. IBM Journal of Research and Development. 2005;49(4.5):755-775.

2. Miller D. Rationale and challenges for optical interconnects to electronic chips. Proceedings of the IEEE. 2000;88(6):728-749.

3. Griese E. A high-performance hybrid electrical-optical interconnection technology for high-speed electronic systems. IEEE Transactions on Advanced Packaging. 2001;24(3):375-383.

4. Forbes M, Gourlay J, Desmulliez M. Optically interconnected electronic chips: a tutorial and review of the technology. Electronics \& Communication Engineering Journal. 2001;13(5):221-232.

5. Berger C, Kossel M, Menolfi C, Morf T, Toifl T, Schmatz M. High-density optical interconnects within large-scale systems. Proceedings of SPIE. 2003;4942:222-235.

6. Beals J, Bamiedakis N, Wonfor A, Penty R, White I, DeGroot J, Clapp T, Glick M. Terabit capacity passive polymer optical backplane. Conference on Lasers and Electro-Optics/Quantum Electronics and Laser Science Conference and Photonic Applications Systems Technologies. 2008;:1-2, 4-9.

7. Dangel R, Berger C, Beyeler R, Dellmann L, Gmur M, Hamelin R, Horst F, Lamprecht T, Morf T, Oggioni S, Spreafico M, Offrein B. Polymer-WaveguideBased Board-Level Optical Interconnect Technology for Datacom Applications. IEEE Transactions on Advanced Packaging. 2008;31(4):759-767.

8. Riegel N. Using the 3D Beam Propagation Method to Model the Effects of Lithographic Roughness on the Attenuation of Multimode Polymer Waveguides. Unpublished.

9. Mohammed E, Alduino A, Thomas T, Braunisch H, Lu D, Heck J, Liu A, Young I, Barnett B, Vandentop G, Mooney R. Optical interconnect system integration for ultra-short-reach applications. Intel Technology Journal. 2004;8(2):115-128.

10. Haaland P, McKibben J, Paradi M. Fundamental Constraints on Thin-Film Coatings for Flat-Panel Display Manufacturing. SID Display Manufacturing Technology Conference. 1995;:79.

11. Haaland P, McKibben J, Parodi M. The art and science of thin-film coating: a progress report. Solid State Technology. 1995;38(4):83.

12. Luurtsema G. Spin coating for rectangular substrates. Masters Thesis, University of California, Berkeley. 1997.

13. Pham N, Burghartz J, Sarro P. Spray Coating of Photoresist for pattern transfer on high topography surfaces. Journal of Micromechanics and Microengineering. 2005;15(4):691-697. 
14. Yu L, Lee YY, Tay FEH, Iliescu C. Spray coating of photoresist for 3D microstructures with different geometries. Journal of Physics: Conference Series. 2006;34(1):776-782.

15. Lee YY, Yu L, Tay FEH, Iliescu C. Optimization of spray coating photoresist for high topography surfaces. Semiconductor Conference, 2005. CAS 2005 Proceedings. 2005 International. 2005;1:171-174.

16. Reighard M, Barendt N. Advancements in Conformal Coating Process Controls. Nepcon West 2000 Conference Proceedings. 2000.

17. Neyer A, Kopetz S, Rabe E, Kang W, Tombrink S. Electrical-Optical Circuit Board using Polysiloxane Optical Waveguide Layer. Electronic Components and Technology Conference Proceedings 2005. 2005;55:246-250.

18. Krebs F. Fabrication and processing of polymer solar cells: A review of printing and coating techniques. Solar Energy Materials and Solar Cells. 2009;93(4):394412.

19. Chappell J, Hutt D, Conway P. Variation in the line stability of an inkjet printed optical waveguide applicable material. Electronics System-Integration Technology Conference, 2008. 2008;(2):1267-1272.

20. Cooley P, Wallace D, Antohe B. Application of ink-jet printing technology to BioMEMS and microfluidic systems. Journal of Laboratory Automation. 2001;7(5):33-39.

21. Calvert P. Inkjet Printing for Materials and Devices. Chemistry of Materials. 2001;13(10):3299-3305.

22. de Gans B, Duineveld P, Schubert U. Inkjet printing of polymers: State of the art and future developments. Advanced Materials. 2004;16(3):203-213.

23. Ishii $\mathrm{Y}$, Koike $\mathrm{S}$, Arai $\mathrm{Y}$, Ando $\mathrm{Y}$. Ink-jet fabrication of polymer microlens for optical-I/O chip packaging. Japanese Journal of Applied Physics. 2000;39(3B):1490-1493.

24. Chen X, Shoenau G, Zhang W. Modeling of time-pressure fluid dispensing processes. IEEE Transactions on Electronics Packaging Manufacturing. 2000;23(4):300-305.

25. Chen X, Kai J. Modeling of positive-displacement fluid dispensing processes. IEEE Transactions on Electronics Packaging Manufacturing. 2004;27(3):157163.

26. Kai J. Modeling of Positive-Displacement Dispensing Process. Masters Thesis. University of Saskatchewan, 2008.

27. Van Steenberge G, Hendrickx N, Bosman E, Van Erps J, Thienpont H and Van Daele P. Laser ablation of parallel optical interconnect waveguides. IEEE Photonics Technology Letters. 2006;18(9):1106-1108.

28. Zakariyah S, Conway P, Hutt D, Selviah D, Wang K, Baghsiahi H, Rygate J, Calver J, Kandulski W. Polymer optical waveguide fabrication using laser ablation. Electronics Packaging Technology Conference, 2009. 2009;11:936-941. 
29. Zakariyah S, Conway P, Hutt D, Selviah D, Wang K, Rygate J, Calver J, Kandulski W. Fabrication of Polymer Waveguides by Laser Ablation Using a $355 \mathrm{~nm}$ Wavelength Nd:YAG Laser. Journal of Lightwave Technology. 2011;29(23):3566-3576.

30. Selviah D. Polymer multimode waveguide optical and electronic PCB manufacturing. Proceedings of SPIE. 2009;7219.

31. Wang X, Jiang W, Wang L, Bi H, Chen R. Fully Embedded Board-Level Optical Interconnects From Waveguide Fabrication to Device Integration. Journal of Lightwave Technology. 2008;26(2):243-250.

32. Missinne J, Van Steenberge G, Van Hoe B, Van Coillie K, Van Gijseghem T, Dubruel P, Vanfleteren J, Van Daele P. An array waveguide sensor for artificial optical skins. Proceedings of SPIE. 2009;7221.

33. Wang L, Wang X, Jiang W, Choi J, Bi H, Chen R. $45^{\circ}$ polymer-based total internal reflection coupling mirrors for fully embedded intraboard guided wave optical interconnects. Applied Physics Letters. 2005;87(14):141110-141110-3.

34. Neyer A, Knoche T, Muller L. Fabrication of low loss polymer waveguides using injection moulding technology, Electronics Letters. 1993;29(4):399-401.

35. Choi CG, Han SP, Kim BC, Ahn SH, Jeong MY. Fabrication of large-core 1 x 16 optical power splitters in polymers using hot-embossing process. IEEE Photonics Technology Letters. 2003;15(6):825-827.

36. Leng Y, Yun V, Lucas L, Herman W, Goldhar J. Dispensed polymer waveguides and laser-fabricated couplers for optical interconnects on printed circuit boards. Applied Optics. 2007;46(4):602-610.

37. Keyworth B, McMullin J, Narendra R,MacDonald R. Computer-controlled pressure-dispensed multimode polymer waveguides. IEEE Transactions on Components, Packaging, and Manufacturing Technology, Part B: Advanced Packaging. 1995;18(3):572-577.

38. Kalajian J. Towards a single-mode dispensed polymer optical waveguide. Masters Thesis. University of South Florida, 2003.

39. Dellman L, Berger C, Beyeler R, Dangel R, Gmur M, Hamelin R, Horst F, Lamprecht T, Meier N, Morf T, Oggioni S, Spreafico M, Stevens R, Offrein B. $120 \mathrm{~Gb} / \mathrm{s}$ Optical Card-to-Card Interconnect Link Demonstrator with Embedded Waveguides. Electronic Components and Technology Conference, 2007 Proceedings. 2007;57:1288-1293.

40. Chen R, Lin L, Choi C, Liu Y, Bihari B, Wu L, Tang S, Wickman R, Picor B, Hibb-Brenner M, Bristow J, Liu Y. Fully embedded board-level guided-wave optoelectronic interconnects. Proceedings of the IEEE. 2000;88(6)780-793.

41. Tooley F, Suyal N, Bresson F, Fritze A, Gourlay J, Walker A, Emmery M. Optically written polymers used as optical interconnects and for hybridisation. Optical Materials. 2001;17(1-2):235-241. 
42. Eldada L, Chengzeng X, Stengel K, Shacklette L, Yardley J. Laser-fabricated low-loss single-mode raised-rib waveguiding devices in polymers. Journal of Lightwave Technology. 1996;14(7):1704-1713.

43. Hwang SH, Lim JW, Lee WJ, Kim GW, Cho CH, An JB, Jung KY, Cha KS, Rho BS. Bendable and Splitter-Integrated Optical Subassembly Based on a Flexible Optical Board. IEEE Photonics Technology Letters. 2010;22(3):167-169.

44. Yoshimura R, Hikita M, Usui M, Tomaru S, Imamura S. Polymeric optical waveguide films with $45^{\circ}$ mirrors formed with a $90^{\circ} \mathrm{V}$-shaped diamond blade. Electronics Letters. 1997:33(15);1311-1312.

45. Matsubara T, Oda K, Watanabe K, Tanaka K, Maetani M, Nishimura Y, Tanahashi S. Three dimensional optical interconnect on organic circuit board. Electronic Components and Technology Conference, 2006 Proceedings. 2006;56:6.

46. Dou X, Wang X, Huang H, Lin X, Ding D, Pan D, Chen R. Polymeric waveguides with embedded micro-mirrors formed by Metallic Hard Mold. Optics Express. 2010;18(1):378-385.

47. Booth BL, Fisher J. Optoelectronics Comes of Age. Printed Circuit Design \& Fab. 2008.

48. Choi C, Lin L, Liu Y, Choi J, Wang L, Haas D, Magera J, Chen R. Flexible optical waveguide film fabrications and optoelectronic devices integration for fully embedded board-level optical interconnects. Journal of Lightwave Technology. 2004;22(9):2168- 2176.

49. Hendrickx N, Van Erps J, Van Steenberge G, Thienpont H, Van Daele P. Laser Ablated Micromirrors for Printed Circuit Board Integrated Optical Interconnections. Photonics Technology Letters. 2007;19(11):822-824.

50. Van Steenberge G, Geerinck P, Van Put S, Van Koetsem J, Ottevaere H, Morlion D, Thienpont H, Van Daele P. MT-compatible laser-ablated interconnections for optical printed circuit boards. Journal of Lightwave Technology. 2004;22(9):2083-2090.

51. Kagami M, Kawasaki A, Ito H. A polymer optical waveguide with out-of-plane branching mirrors for surface-normal optical interconnections. Journal of Lightwave Technology. 2001;19(12):1949-1955.

52. Immonen M, Karppinen M, Kivilahti J. Fabrication and characterization of polymer optical waveguides with integrated micromirrors for three-dimensional board-level optical interconnects. IEEE Transactions on Electronics Packaging Manufacturing. 2005;28(4):304-311.

53. Wang F, Liu F, Adibi A. 45 Degree polymer micro-mirror integration for boardlevel three-dimensional optical interconnects. Electronic Components and Technology Conference, 2009. 2009;59:1842-1845.

54. McCarthy A, Suyal H, Walker A. Fabrication and characterisation of direct laserwritten multimode polymer waveguides with out-of-plane turning mirrors. Conference on Lasers and Electro-Optics Europe, 2005. 2005;:477. 
55. Kim JS, Kim JJ. Fabrication of multimode polymeric waveguides and micromirrors using deep X-ray lithography. IEEE Photonics Technology Letters. 2004;16(3)798-800.

56. Hietala J, Muukkonen E, von Lerber T, Immonen M, Oy A. Volume production of on-board optical waveguides. Electronic Components and Technology Conference, 2005 Proceedings. 2005;55(2):1735-1738.

57. Garner S, Lee SS, Chuyanov V, Chen A, Yacoubian A, Steier W, Dalton L. Three-dimensional integrated optics using polymers. IEEE Journal of Quantum Electronics. 1999;35(8):1146-1155.

58. Karppinen M, Makinen J, Kataja K, Tanskanen A, Alajoki T, Karioja P, Immonen M, Kivilahti J. Embedded optical interconnect on printed wiring board. Proceedings of SPIE Photonics Europe Conference. 2004;5453:150-164.

59. Takahara H. Optoelectronic multichip module packaging technologies and optical input/output interface chip-level packages for the next generation of hardware systems. IEEE Journal of Selected Topics in Quantum Electronics. 2003;9(2):443-451.

60. Karppinen M, Alajoki T, Tanskanen A, Kataja K, Makinen JT, Kautio K, Karioja P, Immonen M, Kivilahti J. Parallel optical interconnect between ceramic BGA packages on FR4 board using embedded waveguides and passive optical alignments. Electronic Components and Technology Conference, 2006 Proceedings. 2006;56:7.

61. Frese I, Schwab U, Nahrstedt E, Klotzbuecher T, Kunz S, Teubner U, Doll T, Polymer based optical interconnect components for high-speed Datacom approaches- Micromachining supported manufacturing. Optics Communications. 2006;265(2):434-440.

62. Van Erps J, Hendrickx N, Debaes C, VanDaele P, Thienpont H. Discrete Out-ofPlane Coupling Components for Printed Circuit Board-Level Optical Interconnections. IEEE Photonics Technology Letters. 2007;19(21):1753-1755.

63. Van Daele P. Optical interconnections for short distances. Proceedings of the Symposium on Photonics Technologies for 7th Framework Program. 2006;:270273.

64. Clay K, Gardner I, Bresler E, Seal M, Speakman S. Direct legend printing (DLP) on printed circuit boards using piezoelectric inkjet technology. Circuit World. 2002;28(2):24-31.

65. Texas Instruments [Internet]. www.ti.com. 2008 August 18 [cited 2012 July 13]. Available:

http://focus.ti.com/pdfs/dlpdmd/Digital_Projection_of_UV_Light_is_Enabling _ New_Markets1.pdf.

66. Jönsson S, Hasselgren C. Secrets of the dark, Fusion UV Systems, Inc. 910 Clopper Road, Gaithersburg MD 20878.

67. Stowe R. Dichroic Reflectors Applied to High Peak Irradiance Microwave Powered UV Lamps. RadTech North America Proceedings. 1992. 
68. Kowalevicz A, Bucholtz F. Beam Divergence from an SMF-28 Optical Fiber, Naval Research Laboratory Washington DC. 2006.

69. Hodgman CD. Handbook of Chemistry and Physics. 20th ed. Cleveland(OH): Chemical Rubber Publishing Company; 1948. p.1742.

70. Bierhoff T, Schrage J, Halter M, Betschon F, Duis J, Rietveld W. All optical pluggable board-backplane interconnection system based on an MPX-FlexTail connector solution. 2010 IEEE Photonics Society Winter Topicals Meeting Series (WTM). 2010;:91-92.

71. Pitwon R, Hopkins K, Wang K, Selviah D, Baghsiahi H, Offrein B, Dangel R, Horst F, Halter M, Gmur M. Design and implementation of an electroopticalbackplane with pluggable in-planeconnectors. Proceedings of SPIE. $2010 ; 7607$.

72. Offrein B, Berger C, Beyeler R, Dangel R, Dellmann L, Horst F, Lamprecht T, Meier N, Budd R, Libsch F, Kash J. Parallel optical interconnects in printed circuit boards. Proceedings of SPIE. 2005;5990.

73. Kruse K, Riegel N, Demars C, Middlebrook C, Roggeman M. Optical waveguide end roughness in correlation to optical coupling. Proceedings of SPIE. $2012 ; 8267$.

74. Immonen M, Karppinen M, Kivilahti J. Investigation of Environmental Reliability of Optical Polymer Waveguides Embedded on Printed Circuit Boards. Microelectronics Reliability. 2007;47(2-3):363-371.

75. Abernethy R. The New Weibull Handbook. 5th ed. North Palm Beach(FL): R.B. Abernethy; 1993. 


\section{APPENDIX A: SUMMARY TABLE OF MTU LASER WRITING PARAMETERS AND RESULTS}

Table 7.1

Laser direct writing parameters and results for MTU focused laser diode module, characterizing standoff-distance and energy dose.

\begin{tabular}{|c|c|c|c|c|}
\hline \multicolumn{2}{|c|}{ Setting } & \multirow{2}{*}{$\begin{array}{c}\text { Width (Axis 1) } \\
\text { AVG } \\
(\mu \mathrm{m})\end{array}$} & \multirow{2}{*}{$\begin{array}{c}\text { Width (Axis 2) } \\
\text { AVG } \\
(\mu \mathrm{m})\end{array}$} & \multirow{2}{*}{$\begin{array}{c}\text { Axis Comparison } \\
\text { Size Difference } \\
\% \\
\end{array}$} \\
\hline $\begin{array}{l}\text { Standoff } \\
\text { Distance } \\
(\mathrm{mm})\end{array}$ & $\begin{array}{c}\text { Dose } \\
\left(\mathrm{mJ} / \mathrm{cm}^{2}\right)\end{array}$ & & & \\
\hline 43 & 2400 & 79.7 & 91.3 & 13.6 \\
\hline 43 & 1800 & 74.2 & 87.3 & 16.2 \\
\hline 43 & 1500 & 73.8 & 80.4 & 8.6 \\
\hline 43 & 1200 & 68.8 & 77.0 & 11.1 \\
\hline 43 & 900 & 60.2 & 70.5 & 15.9 \\
\hline 43 & 600 & 50.1 & 61.9 & 21.2 \\
\hline 44 & 2400 & 70.6 & 70.3 & 0.4 \\
\hline 44 & 1800 & 62.9 & 62.2 & 1.2 \\
\hline 44 & 1500 & 60.2 & 60.9 & 1.3 \\
\hline 44 & 1200 & 53.0 & 60.2 & 12.6 \\
\hline 44 & 900 & 48.6 & 56.0 & 14.2 \\
\hline 44 & 600 & 40.2 & 45.6 & 12.6 \\
\hline 45 & 2400 & 74.0 & 68.8 & 7.3 \\
\hline 45 & 1800 & 62.7 & 54.5 & 13.9 \\
\hline 45 & 1500 & 60.4 & 52.3 & 14.4 \\
\hline 45 & 1200 & 50.6 & 49.8 & 1.5 \\
\hline 45 & 900 & 44.2 & 45.6 & 3.3 \\
\hline 45 & 600 & 35.5 & 31.1 & 13.4 \\
\hline 46 & 2400 & 80.4 & 90.3 & 11.6 \\
\hline 46 & 1800 & 67.1 & 71.1 & 5.8 \\
\hline 46 & 1500 & 60.4 & 66.4 & 9.4 \\
\hline 46 & 1200 & 53.8 & 51.8 & 3.8 \\
\hline 46 & 900 & 41.9 & 35.5 & 16.6 \\
\hline 46 & 600 & 32.3 & 31.1 & 3.9 \\
\hline 47 & 2400 & 87.6 & 119.9 & 31.2 \\
\hline 47 & 1800 & 72.8 & 90.8 & 22.0 \\
\hline 47 & 1500 & 66.3 & 77.9 & 16.0 \\
\hline 47 & 1200 & 59.9 & 70.3 & 15.9 \\
\hline 47 & 900 & 52.8 & 51.5 & 2.4 \\
\hline 47 & 600 & 42.4 & 37.5 & 12.4 \\
\hline
\end{tabular}




\section{APPENDIX B: PERMISSIONS FROM PUBLISHERS}

Permission for Figure 3.5

Hi Joe,

Thanks for your patience. Attached is the cleared absorption spectrum for you to use in your dissertation.

Congrats again (on multiple fronts)!

Cheers,

dave

David Deshazer

Dow Corning Corp. 